University of Louisville

ThinkIR: The University of Louisville's Institutional Repository

Electronic Theses and Dissertations

$5-2012$

\title{
Trapping of nanoparticles with dielectrophoretic nano-probes.
}

Nicholas Wood

University of Louisville

Follow this and additional works at: https://ir.library.louisville.edu/etd

\section{Recommended Citation}

Wood, Nicholas, "Trapping of nanoparticles with dielectrophoretic nano-probes." (2012). Electronic Theses and Dissertations. Paper 1588.

https://doi.org/10.18297/etd/1588

This Master's Thesis is brought to you for free and open access by ThinkIR: The University of Louisville's Institutional Repository. It has been accepted for inclusion in Electronic Theses and Dissertations by an authorized administrator of ThinkIR: The University of Louisville's Institutional Repository. This title appears here courtesy of the author, who has retained all other copyrights. For more information, please contact thinkir@louisville.edu. 
By

Nicholas Wood

Bachelor of Science in Mechanical Engineering, University of Louisville, 2011

\author{
A Thesis \\ Submitted to the Faculty of the \\ University of Louisville \\ J.B. Speed School of Engineering \\ as Partial Fulfillment of the Requirements \\ for the Professional Degree
}

MASTER OF ENGINEERING

Department of Mechanical Engineering

May 2012 
TRAPPING OF NANOPARTICLES WITH DIELECTROPHORETIC NANO-PROBES

Submitted by:

Nicholas R. Wood

A Thesis Approved On

(Date)

by the Following Reading and Examination Committee:

Dr. Stuart J. Williams, Thesis Director

Dr. Robert W. Cohn

Dr. Thomas A. Berfield 


\section{ACKNOWLEDGMENTS}

I would not have been able to complete this thesis without the help of my thesis director, Dr. Stuart Williams, or my wife, Erica Wood. I cannot understate the amount of support, both academic and moral, that they provided to me while writing this thesis. Additionally, I would like to thank the other people that work in the SRB answered my questions or trained me on equipment. Specifically, Joe Williams for all of his help with the imaging of the nano-probes, Mark Crain for answering general questions, and Don Yeager for training me on the equipment in the cleanroom. 


\begin{abstract}
Dielectrophoresis (DEP) is an electrokinetic force capable of attracting or repelling neutrally charged particles due to a non-uniform electric field [1, 2]. Positive dielectrophoresis attracts particles in the region of the highest electric field gradient; negative dielectrophoresis repels particles from the region of the highest electric field gradient. The dielectrophoretic force is directly proportional to the square of the electric field gradient, as well as the cube of the radius of the particles involved.

As particles decrease in size, the gradient of the electric field must increase rapidly in order to capture or repel the particles. The intense electric field gradients were produced using fabricated silver gallium $\left(\mathrm{Ag}_{2} \mathrm{Ga}\right)$ nano-probes electrodes in conjunction with indium tin oxide (ITO) coated microscope cover slips, which served as the opposite electrode. The silver gallium nano-probes ranged from approximately $100-500 \mathrm{~nm}$ in diameter and were typically positioned less than $40 \mu \mathrm{m}$ above the ITO cover slips. Positive and negative dielectrophoretic forces were able to dominate the other electrokinetic forces acting on sub-micron particles, which were suspended in deionized water and aqueous potassium chloride, using the nano-probes and ITO cover slips as electrodes. Colloidal quantum dots of gold, as small as $5 \mathrm{~nm}$ in diameter, were captured using positive DEP forces, as were sub-micron fluorescent polystyrene particles. Negative DEP forces repelled sub-micron fluorescent polystyrene particles suspended in a low conductivity solution.
\end{abstract}


TABLE OF CONTENTS

APPROVAL PAGE iii

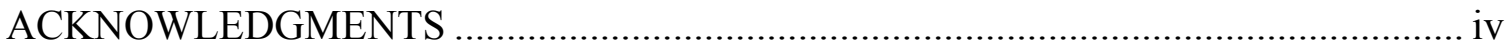

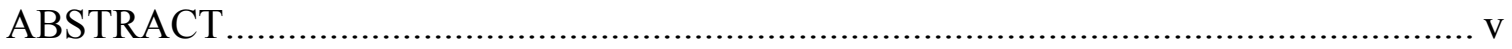

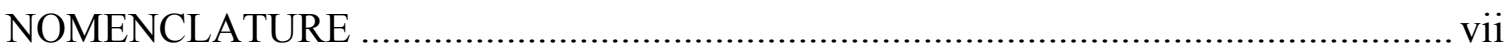

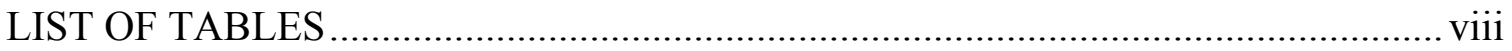

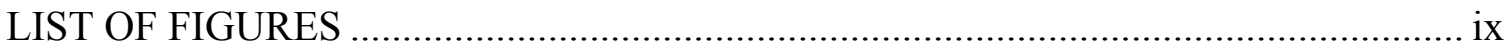

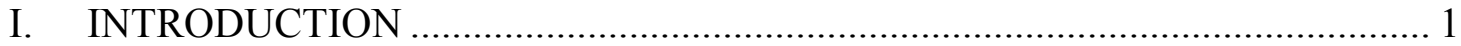

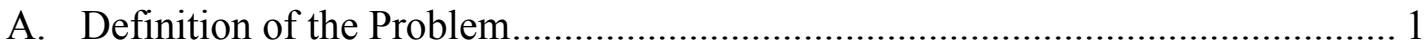

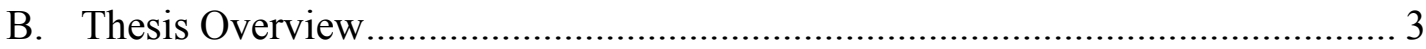

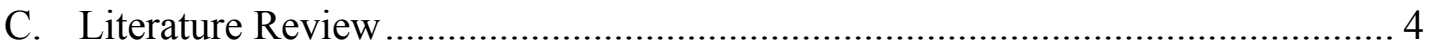

1. Gravitational Force and Stokes Drag.......................................................... 4

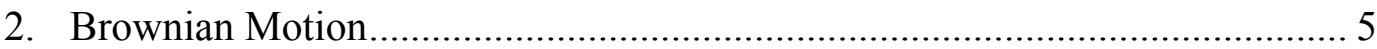

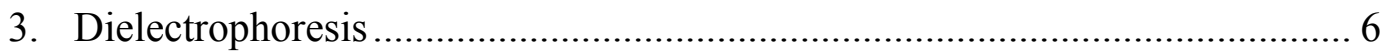

4. Electrohydrodynamic Forces ................................................................... 17

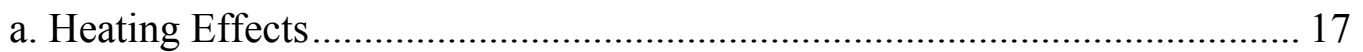

b. Electrothermal Hydrodynamics ................................................................. 20

5. Total Particle Motion - An Overview ……….............................................. 22

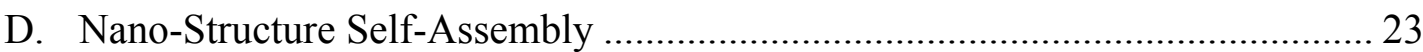

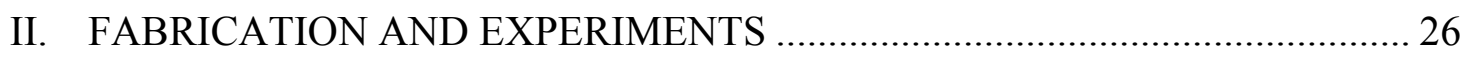

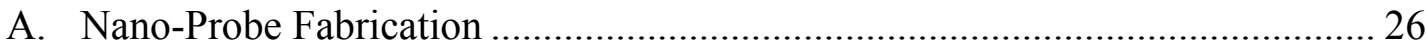

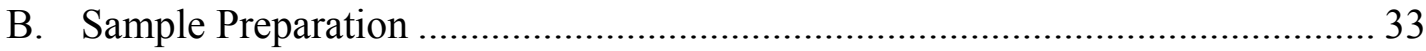

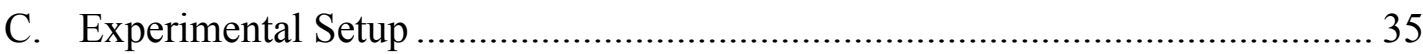

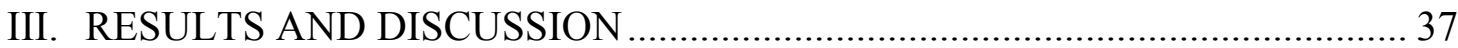

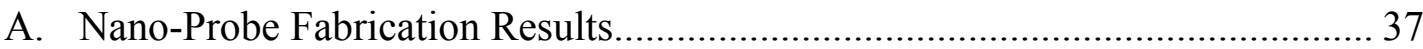

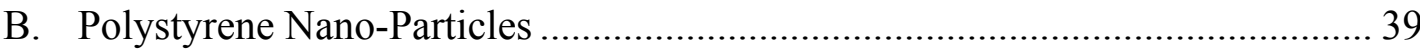

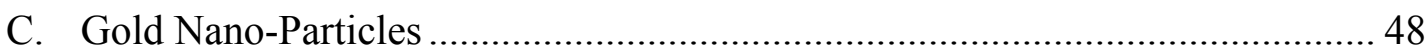

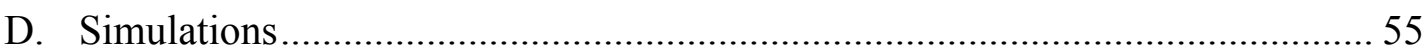

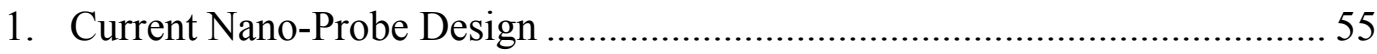

2. Revised Nano-Probe Designs ..................................................................... 58

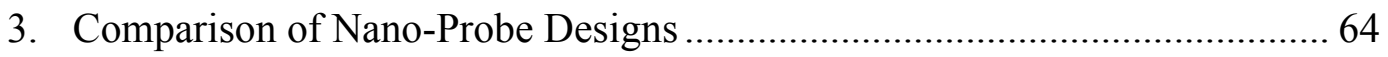

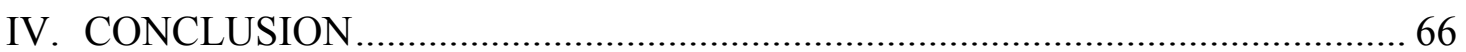

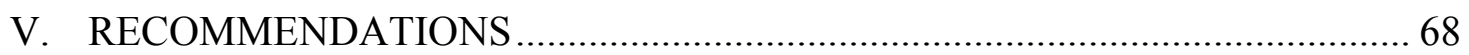

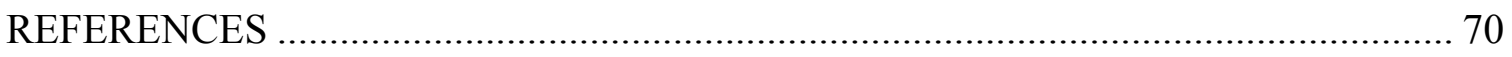

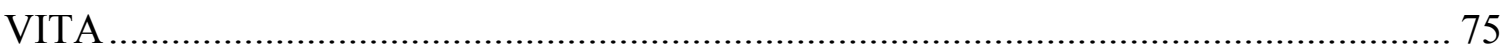




\section{NOMENCLATURE}

$C M$ - Clausius-Mossotti factor

$c_{p}$ - specific heat of the medium

$D$ - diffusion coefficient

$d_{p}$ - diameter of the particle

$E_{r m s}-$ RMS value of the electric field

$E_{x}(t)$ - time dependent electric field in the $\mathrm{x}$ direction

$E_{y}(t)$ - time dependent electric field in the y direction

$E_{z}(t)$ - time dependent electric field in the $\mathrm{z}$ direction

$\epsilon_{m}$ - permittivity of the medium

$\epsilon_{p}$ - permittivity of the particle

$\boldsymbol{F}$ - sum of external forces

$\left\langle F_{D E P}\right\rangle$ - time averaged DEP force

$g$ - acceleration due to gravity

$G$ - conductance of the electrolyte

$H(\phi)$ - Hessian matrix of electric potential

$L$ - length between electrodes

$L_{e}$ - characteristic length of electrode

$k_{B}$ - Boltzmann Constant

$K_{s}$ - surface conductance

$k_{m}$ - thermal conductivity of the medium

$m(t)$ - time dependent dipole moment

$\Gamma$-geometry factor

$l$ - length of cylindrical rod

$\psi$ - force on the dipole

$t_{\text {diff }}$ - time to equilibrium temperature

$P$ - Joule heating power $\phi-$ potential

$\mu_{m}$ - viscosity of the medium

$m_{p}-$ particle mass

$\eta$ - friction factor

$r$ - distance between electrodes

$r_{c}$ - radius of curvature of the electrodes

$r_{p}-$ particle radius

$\rho_{m}$ - density of the medium

$\rho_{p}$ - density of the particle

$\sigma_{m \text {,mean }}-$ average conductivity of the medium

$\sigma_{m, 298}$ - conductivity of the medium at $298 \mathrm{~K}$

$T_{\text {mean }}$ - average temperature

$\gamma_{1}, \gamma_{2}$ - temperature coefficients

$\sigma_{b}$ - bulk conductivity of the particle

$\sigma_{m}$ - conductivity of the medium

$\sigma_{p}$ - conductivity of the particle

$\rho_{q}-$ charge density

$t$ - time

$T$ - temperature

$\tau_{M W}-$ Maxwell-Wagner charge

relaxation

$\boldsymbol{u}$ - particle velocity

$V$ - separation voltage

$v$-particle volume

$V_{r m s}-$ RMS value of the potential

$w$ - fluid velocity

$\zeta$ - zeta potential

$\omega-$ frequency $(\mathrm{Hz})$ 


\section{LIST OF TABLES}

TABLE I - LASER SETTINGS FOR SHARP PROBE CREATION …………………... 27

TABLE II - EXPERIMENTAL RESULTS ................................................................ 41

TABLE III - DOMINANT FORCES ACTING ON 200 NM POLYSTYRENE

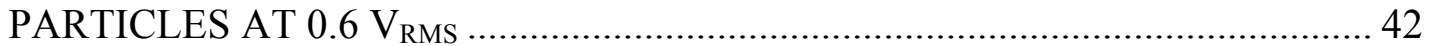

TABLE IV - REQUIRED ELECTRIC FIELD INCREASE FOR DECREASING

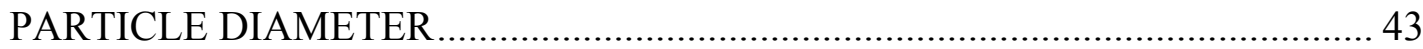

TABLE V - POLYSTYRENE NANO-PARTICLE MOTION DUE TO ET FLOW...... 45

TABLE VI - CHARACTERISTICS OF REVISED NANO-PROBES AT V $=1 \mathrm{~V}_{\mathrm{RMS}}$

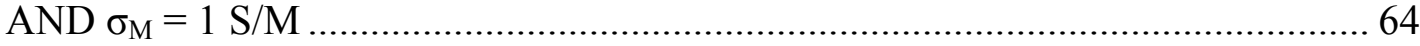




\section{LIST OF FIGURES}

FIGURE 1 - Electric Field Gradient Between a Point Source and a Flat Plane ................ 7

FIGURE 2 - Tri-Axial AFM Probe [63] ……………............................................. 13

FIGURE 3 - Sutter Instruments Micro-Pipette Puller................................................... 27

FIGURE 4 - Sharp Probe Pulled in the Sutter Instruments P-2000 Micro-Pipette Puller 28

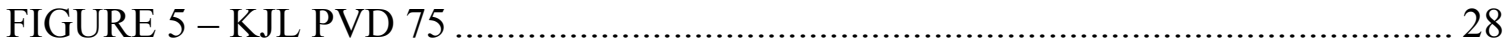

FIGURE 6 - Microscope for Viewing Nano-Probe Creation........................................... 30

FIGURE 7 - A) The Piezo-PatchTM PPM5000 Micro-Controller. B) The Piezo-PatchTM PPM 5000 Nano-Manipulator........................................................................... 31

FIGURE 8 - Diagram of the Nano-Probe Pulling Process: (A) The Reverse Meniscus, (B) Nano-probe Formation, (C) Glass Capillary Tube Withdrawal, and (D) NanoProbe Attached to Capillary Tube.

FIGURE 9 - Typical Nano-Probes. (A) Conductive Layers of Silver Separated by Glass Does Not Permit a Signal to be Sent to the Nano-Probe, (B) Conductive Layers of Silver Separated by Glass, but Coated in Gold to Permit Electron Movement ........ 33

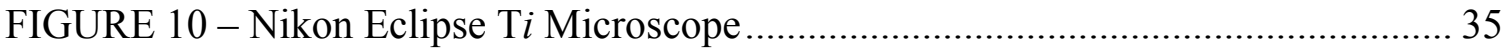

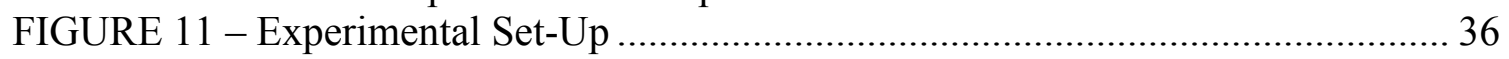

FIGURE 12 - Olympus IX71 Microscope ……………............................................ 36

FIGURE 13 - Reflected Image of a Micro-Electrode and Nano-Probe. $260 \mathrm{~nm}$ in

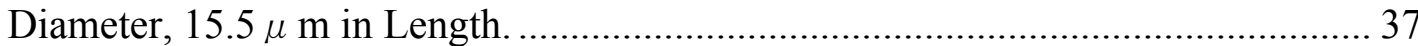

FIGURE 14 - Reflected Image of a Nano-Probe on a Micro-Electrode With a Gallium Droplet. The Nano-Probe is $500 \mathrm{~nm}$ in Diameter, $25 \mu \mathrm{m}$ in Length. .................... 38

FIGURE 15 - Reflected Image of a Nano-Probe. $150 \mathrm{~nm}$ in diameter, $6.1 \mu \mathrm{m}$ in length. 38

FIGURE 16 - SEM Image of a Gold Coated Nano-Probe. $162 \mathrm{~nm}$ in Diameter, $7.96 \mu \mathrm{m}$

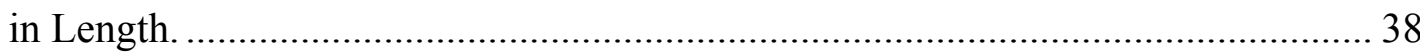

FIGURE 17 - SEM Image of a Gold Coated Nano-Probe. $475 \mathrm{~nm}$ in Diameter, $11.8 \mu \mathrm{m}$ in Length. 38

FIGURE 18 - Time Lapse Sequence of the pDEP Capture and Release of $200 \mathrm{~nm}$ Polystyrene Particles Suspended in DI Water. The $\Delta \mathrm{t}$ Between Photos is $0.725 \mathrm{~s}$. The AC Signal had an Amplitude of $0.6 \mathrm{~V}_{\mathrm{rms}}$ and a Frequency of $600 \mathrm{kHz}$............ 40 FIGURE 19 - To the Left, No Signal is Applied to the Nano-Probe. To the Right, nDEP Particle Repulsion of $200 \mathrm{~nm}$ Polystyrene Particles Suspended in Water with a Conductivity of 5.5E-8 S/m. The AC Signal had an Amplitude of $6 \mathrm{~V}_{\mathrm{rms}}$ and a

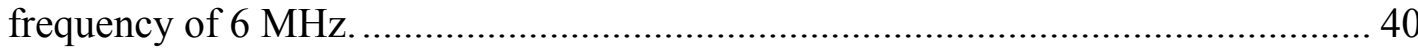

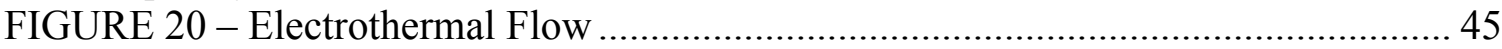

FIGURE 21 - CM Factor as a Function of Frequency for Polystyrene Particles

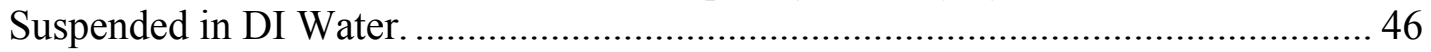

FIGURE 22 - CM Factor as a Function of Frequency for Polystyrene Particles Suspended in a Low Conductivity Solution.............................................................. 47

FIGURE 23 - CM Factor as a Function of Frequency for Polystyrene Particles Suspended in a High Conductivity Solution............................................................. 47

FIGURE 24 - Nano-Probe with Gold Nano-Shells Adhered by pDEP............................. 49

FIGURE 25 - Enlarged View of Nano-Probe and Attached Gold Nano-Shells .............. 49

FIGURE 26 - Gold Nano-Particles Grown on Capillary Tube and Nano-Probe .............. 50 
FIGURE 27 - Dual Nano-Probes with Gold Nano-Particles Grown

FIGURE 28 - Gold Nano-Particle Bridge Between 2 Nano-Probes. The Bridge is Outlined in Black to Enhance Contrast..................................................................... 51

FIGURE 29 - Gold Nano-Particle Bridge Between Two Nano-Probes........................... 52

Figure 30 - SEM Image of Gold Nano-Particles Captured by DEP ............................... 53

Figure 31 - SEM Image of a Gold Nano-Particle Cluster ............................................... 53

Figure 32 - SEM Image of Gold Nano-Particles Fused to the Nano-Probe by pDEP ..... 54

FIGURE 33 - Gold Nano-Particles Adhered to Nano-Probe by pDEP............................ 54

FIGURE 34 - Enlarged View of Gold Nano-Particles Adhered to Nano-Probe by pDEP

FIGURE 35 - Schematic of Nano-Probe and ITO Coverslip with Boundary Conditions 56

FIGURE 36 - Maximum Temperature Increase as a Function of Voltatge and the Conductivity of the Medium............................................................................. 56

FIGURE 37 - Maximum Temperature Gradient as a Function of Voltage and the Conductivity of the Medium 57

FIGURE 38 - Trapping Radius as a Function of Applied Voltage ................................ 57

FIGURE 39 - pDEP Near the Tip of the Nano-Probe. $0.707 \mathrm{~V}_{\text {rms }}, 1 \mathrm{kHz}$..................... 58

FIGURE 40 - nDEP Near the Tip of the Nano-Probe. $0.707 \mathrm{~V}_{\mathrm{rms}}, 10 \mathrm{MHz}$.................. 58

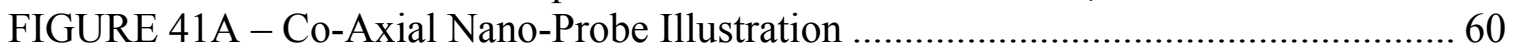

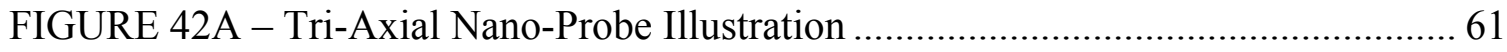

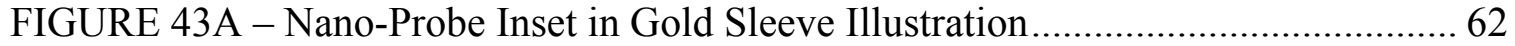

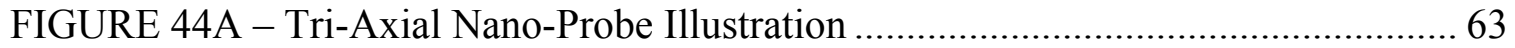




\section{INTRODUCTION}

\section{A. Definition of the Problem}

The nano-sciences have been of particular interest ever since Richard Feynman's revolutionary speech in 1959 [3], "There's Plenty of Room at the Bottom." In his speech, Feynman proposed that entire libraries could be printed on index cards, and discussed the process of arranging individual atoms [3]. While researchers may not yet be capable of manipulating individual atoms on the scale that Feynman proposed, his proposal laid the groundwork for what is now known as top-down manufacturing. The self-assembly, or spontaneous formation, of nano-structures has the potential to greatly benefit top-down manufacturing [4]. Self-assembly is one of the more interesting of the manufacturing methods used to manufacture nano-structures, but it has its own challenges. The photolithographic processing of silicon wafers is a more traditional method used to manufacture nano-structures. Nano-structures, regardless of the assembly process, have proved to be very beneficial to society in a variety of applications. Transistors are one of the most obvious examples of the importance of nano-structures [5]. Other beneficial applications of nano-technologies include lab-on-a-chip [6] and air filtration [7].

Both lab-on-a-chip and air filtration technologies require the manipulation of nanosized particles. The ability to manipulate nano-sized particles is influenced by a variety 
of sources such as Brownian motion [8], Stokes drag [9], and the externally applied forces. In particular, electrokinetic methods have demonstrated successful colloid assembly. The manipulation of fluids or particles with electric fields is known as electrokinetics.

Electrokinetic forces at the microscale are capable of generating fluid motion and particle translation. AC electro-osmosis, Joule heating, and electrothermal fluid flow are all examples of electrokinetic hydrodynamic phenomena. In general, electrokinetic particle manipulation is achieved using electrophoresis or dielectrophoresis. Electrophoresis has been widely studied [10] and is understood to be the force acting on a charged particle in a DC electric field. The experiments herein do not deal with the electrophoretic forces, but rather with the other type of electrokinetic particle manipulation - dielectrophoresis. Dielectrophoresis (DEP) is an electrokinetic force which has demonstrated the ability to trap, sort, and manipulate biological entities $[1,11$ $23]$, as well as other materials such as gold [5, 24-32], and polystyrene or latex [6, 14, 16, 18, 19, 23, 33-43]. DEP was discovered by Herbert Pohl in the 1960s [1].

Thus far, the ability to manipulate and measure sub-cellular matter inside living cells in real-time has not been widely studied [44]. Several complications exist when attempting to manipulate and measure sub-cellular matter. Perhaps the most glaring obstacle that must be overcome is that living cells can only be studied under an optical light microscope. The light microscope has a theoretical limit that approaches the wavelength of red light $(330 \mathrm{~nm})$, although microscopes with high numerical apertures can resolve $250 \mathrm{~nm}$ features or even slightly smaller [44, 45]. Resolving power is not the only issue to manipulating sub-cellular entities in living cells. In order to manipulate the 
sub-cellular entities using electrokinetic forces inside of a living cell, an electrode needs to be placed inside the cellular wall or membrane, and the electrode must be inserted in a manner that will not rupture the cellular membrane. For this to be possible, an electrode with a very high aspect ratio must be used. This high aspect ratio electrode must be rigid enough to withstand the associated mechanical forces from a variety of sources.

The future goal of this work is to manipulate sub-cellular entities using DEP forces. The DEP force as well as its magnitude and direction depend heavily on the conductivity and permittivity of the suspended particles, as well as the conductivity and permittivity of the solution. In living cells, the sub-cellular entities are treated as the particles in solution and the cytoplasm in the cell is treated as the fluid medium.

\section{B. Thesis Overview}

This work documents the preliminary proof-of-concept investigation of the manipulation and measurement of sub-cellular entities. Non-biological nanometer-sized polystyrene and gold particles were captured by gold coated silver gallium nano-probes for solutions of various conductivities, $5.5 \mathrm{E}-8 \mathrm{~S} / \mathrm{m}, 2.5 \mathrm{E}-3 \mathrm{~S} / \mathrm{m}$ and $2.26 \mathrm{E}-1 \mathrm{~S} / \mathrm{m}$, proving the . This work also contains numerical simulations of both positive and negative DEP forces for different nano-probe geometries and electrode configurations in order to gain insight into future designs.

This preliminary investigation provides the first step in developing a device to manipulate and measure sub-cellular entities inside living cells by using DEP forces. This project uses a moveable nano-probe to capture the sub-micron, sub-cellular entities. While the work herein does not cover the capture of these sub-cellular entities, it does 
prove that the DEP capture of sub-micron particles is possible using a movable silver gallium electrode.

\section{C. $\quad$ Literature Review}

Particles suspended in a solution are subjected to numerous external forces. Some forces can be attributed to the presence of an applied electric field, while others are due to random molecular motion [9]. These forces are often non-linear with respect to the characteristics of the system. The following section briefly discusses a few of the dominant forces in the system.

\section{Gravitational Force and Stokes Drag}

Stokes drag is an important parameter to consider when attempting to quantify how particles move in a fluid medium [9]. Particles experience a drag force due to the viscosity and relative velocity of the medium. Applying Newton's Second Law to the system, the equation of motion for a particle can be expressed as

$$
m_{p} \dot{\boldsymbol{u}}=-\eta(\boldsymbol{u}-\boldsymbol{w})+\boldsymbol{F}
$$

where $m_{p}$ is the mass of the particle, $\dot{\boldsymbol{u}}$ is the time derivative of the particle's velocity, $\eta$ is the friction factor, $\boldsymbol{u}$ is the velocity of the particle, $\boldsymbol{w}$ is the fluid velocity, and $\boldsymbol{F}$ is the sum of the external forces acting on the particle [9]. The friction factor for spherical particles can be expressed as

$$
\eta=6 \pi r_{p} \mu
$$

where $\mu$ is the viscosity of the medium. Assuming the vector $\boldsymbol{F}$ is a constant, particles less than $1 \mu \mathrm{m}$ in diameter reaches their terminal velocity in a time of less than $1 \mu \mathrm{s}[9]$. 
The terminal velocity of the particles due to the relative motion between the fluid medium and the particle can be expressed as

$$
\boldsymbol{u}=\boldsymbol{w}+\frac{\boldsymbol{F}}{\eta}
$$

Gravity is another force which acts on the suspended particles [9]. The gravitational force on a spherical particle, $F_{g}$, is

$$
F_{g}=\frac{4 \pi}{3} r_{p}^{3} *\left(\rho_{p}-\rho_{m}\right) g
$$

where $r_{p}$ is the radius of the particle, $\rho_{p}$ and $\rho_{m}$ are the density of the particle and medium, respectively, and $g$ is the acceleration due to gravity. The magnitude of the velocity due to gravity, including the Stokes drag, for spherical particles, $u_{g}$, is

$$
u_{g}=\frac{2 r_{p}^{2}\left|\rho_{p}-\rho_{m}\right| g}{9 \mu_{m}}
$$

where $\mu_{m}$ is the viscosity of the medium [9].

\section{Brownian Motion}

Brownian motion, first described by Einstein [8], explains the random molecular motion of particles due to thermal activity. In order to cause this motion, a force must be applied. The force that causes Brownian motion, $F_{B}$, can be expressed as

$$
F_{B}=k_{B} \frac{T}{v^{1 / 3}}
$$

where $k_{B}$ is the Boltzmann constant, $T$ is the temperature, and $v$ is the volume of the particle [43]. This force is independent of direction, and the time averaged Brownian force is zero [9]. A better method of understanding Brownian motion is by observing the particle displacement. Particle displacement due to Brownian motion follows a Gaussian distribution profile. The root mean square of the particle displacement, $x_{r m s}$, is given by 


$$
x_{r m s}=\sqrt{2 D t}
$$

where $D$ is the diffusion coefficient, and $t$ is time. The diffusion coefficient is given by the Stokes-Einstein equation [46, 47]

$$
D=\frac{k_{B} T}{6 \pi \mu_{m} d_{p}}
$$

where $d_{p}$ is the diameter of the particle.

\section{Dielectrophoresis}

Dielectrophoresis (DEP) has been studied extensively since Pohl [1] discovered the phenomenon in the 1960s. Pohl's initial research used a variety of electrode geometries, including a parallel wire setup. Pohl's revolutionary work laid the foundation for future DEP research. However, Pohl did not believe the capture of sub-micron particles would be possible due to the electric field strength required to overcome Brownian motion. Pohl's thoughts regarding DEP on sub-micron particles was disproved in the mid 1990's when sub-micron particles were successfully captured using DEP [48, 49].

After Pohl, one of the next major milestones in understanding the DEP force came in 1995, when Jones published his book, Electromechanics of Particles [2]. This work thoroughly details the mathematics behind the DEP force. Still, this work does not address how DEP forces affect sub-micron particles. After Jones' book, several articles dealing with scaling laws by Castellanos, Ramos, Gonzalez, Green and Morgan [9, 16, 50-53] were published. These articles quantified how the presence of electric fields altered fluid flow due to heating and electric forces. The books and articles mentioned above, along with many others, created the foundation for the studies in this work. The understanding of the DEP and other electrokinetic forces is requisite in order to interpret experimental results herein. 
If a polarizable particle is placed in a non-uniform AC electric field, the particle will experience dielectrophoresis, regardless of the initial polar state of the particle. The nonuniform gradient of the electric field induced a dipole moment in the particles $[1,2]$. Once the dipole moment has been induced, the particle is either attracted or repelled from the region of the sharpest gradient in the electric field. The force responsible for attracting or repelling the particle is called the dielectrophoretic (DEP) force. The DEP force can be positive (pDEP) or negative (nDEP). The pDEP force attracts the particles toward the region with the largest gradient in the electric field, while the nDEP force repels the particles from the largest gradient in the electric field $[1,2,54]$. Figure 1 illustrates the gradient in the electric field as well as the polarization of a neutrally charged body and the subsequent positive dielectrophoretic force $[1,2,54]$ for the basic point-and-plane electrode system with an applied AC signal.

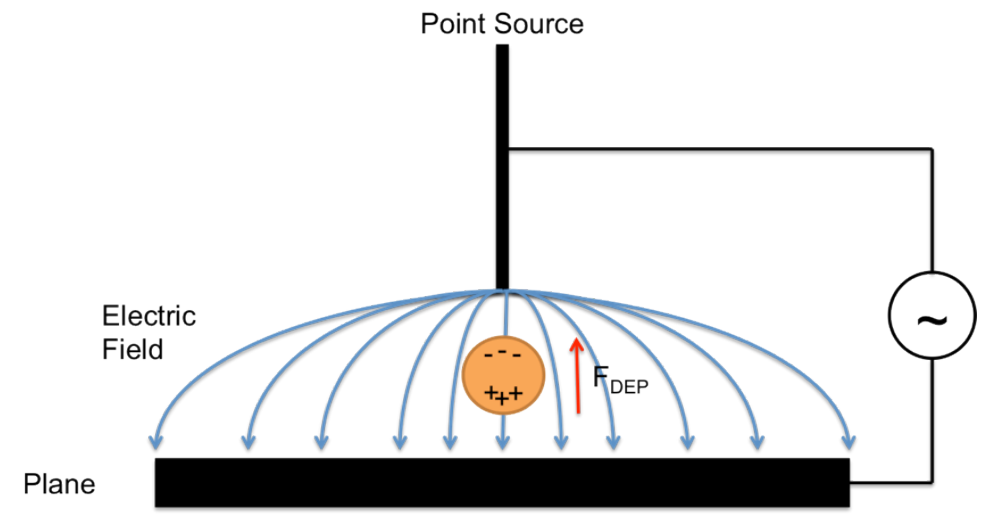

FIGURE 1 - Electric Field Gradient Between a Point Source and a Flat Plane

DEP studies have been conducted using planar electrodes fabricated on silicon substrates $[9,12-14,16,18,20-23,25,30,38,54-62]$. These studies analyzed many different aspects and characteristics of the DEP forces. These studies looked at the pDEP and nDEP forces acting on sub-micron particles made of materials such as latex or 
polystyrene $[18,38]$, or noble metals and their compounds, such as gold and gold sulfide $\left(\mathrm{Au}_{2} \mathrm{~S}\right)[5,18,25-27,29,30,55,58,59]$. Another interesting research area is the field of insulator based DEP (iDEP). iDEP uses two electrodes that create a uniform electric field, then uses dielectric pillars to disrupt the electric field lines, thereby creating a gradient in the electric field [37]. These studies show that there are a variety of different techniques to control, capture, and/or manipulate particles of different shapes, sizes and compositions.

The equation for the DEP force can be easily evaluated for a homogeneous sphere. The DEP force acting on the individual particles increases with the square of the gradient of the applied electric field, and the volume of the particles. The classical, time-averaged DEP force, $\left\langle F_{D E P}\right\rangle$, for homogenous spherical particles experiencing an induced dipole is given by

$$
\left\langle F_{D E P}\right\rangle=2 \pi \varepsilon_{p} \varepsilon_{m} r_{p}^{3} \operatorname{Re}[\mathrm{CM}] \nabla E_{r m s}^{2}
$$

where $\varepsilon_{p}$ and $\varepsilon_{m}$ are the permittivities of the particle and medium, respectively. The variable $r_{p}$ is the radius of the particle, $\mathrm{CM}$ is the Clausius-Mossotti factor, and $\nabla E_{r m s}$ is the gradient of the root mean square of the electric field $[1,2,12-16,18-23,25,30,37$, 38, 54-66]. The root mean square of the gradient of the electric field can be approximated as

$$
\nabla E_{r m s}^{2} \approx \frac{2 r_{c}^{2} c_{r m s}^{2}}{r^{5}} \hat{r}
$$

where $r_{c}$ is the radius of curvature of the electrode, $V_{r m s}$ is the applied voltage, $r$ is the distance between electrodes, and $\hat{r}$ is the unit vector in the radial direction. The $\mathrm{CM}$ factor can be expressed as 


$$
\mathrm{CM}=\frac{\varepsilon_{p}^{\prime}-\varepsilon_{m}^{\prime}}{\varepsilon_{p}^{\prime}+2 \varepsilon_{m}^{\prime}}
$$

where $\varepsilon_{p}^{\prime}$ and $\varepsilon_{m}^{\prime}$ are the complex permittivities of the particle and the medium, respectively $[1,2,6,21,23,54]$. The complex permittivity, $\varepsilon^{\prime}$, is given by

$$
\varepsilon^{\prime}=\varepsilon-i \frac{\sigma}{\omega}
$$

where $\sigma$ is the conductivity, $i=\sqrt{-1}$, and $\omega$ is the angular frequency. $\omega$ is given by

$$
\omega=2 \pi f
$$

where $f$ is the applied frequency $[6,21,23,38]$.

As previously mentioned, pDEP forces occur when particles are attracted to the highest gradient in the electric field. Likewise, nDEP forces are displayed when particles are repelled by the gradient in the electric field. The directionality of the DEP force is governed by the $\mathrm{CM}$ factor, given in equation 11. The $\mathrm{CM}$ factor is controlled by the permittivity of the medium and the particle. The total $\mathrm{Re}[\mathrm{CM}]$ factor can be expressed as

$$
\operatorname{Re}[\mathrm{CM}]=\frac{\varepsilon_{p}-\varepsilon_{m}}{\varepsilon_{p}-2 \varepsilon_{m}}+\frac{3\left(\varepsilon_{m} \sigma_{p}-\varepsilon_{p} \sigma_{m}\right)}{\tau_{M W}\left(\sigma_{p}+2 \sigma_{m}\right)^{2}\left(1+\omega^{2} \tau_{M W}^{2}\right)}
$$

where the subscripts $p$ and $m$ denote the particle and medium, respectively, and $\tau_{\mathrm{Mw}}$ is the Maxwell-Wagner charge relaxation [2] which can be expressed by

$$
\tau_{M W}=\frac{\varepsilon_{p}+\varepsilon_{m}}{\sigma_{p}+2 \sigma_{m}}
$$

The real part of the CM factor at low frequencies, or where $f \cdot \tau_{\mathrm{MW}} \ll 1$, is given by

$$
\operatorname{Re}[\mathrm{CM}]=\frac{\sigma_{p^{-}} \sigma_{m}}{\sigma_{p}+2 \sigma_{m}}
$$

The real part of the CM factor at high frequency, or where $f \cdot \tau_{\mathrm{mw}} \gg 1$, is given by

$$
\operatorname{Re}[\mathrm{CM}]=\frac{\varepsilon_{p}-\varepsilon_{m}}{\varepsilon_{p}+2 \varepsilon_{m}}
$$


The sign of the CM factor determines if $\mathrm{pDEP}$ or $\mathrm{nDEP}$ occurs. A positive $\mathrm{CM}$ factor, i.e. when the polarizibility of the particle is greater than the polarizibility of the medium, indicates pDEP. A negative $\mathrm{CM}$ factor, i.e. when the polarizibility of the particle is less than the polarizibility of the medium, will force the system to experience nDEP [2]. The polarizibility of a suspended polystyrene particle, and hence the CM factor, is frequency dependent [9].

Equations 14 - 17 prove that the $\mathrm{CM}$ factor varies with frequency from a theoretical maximum of 1.0 to a theoretical minimum of -0.5 . Subsequently, depending on the frequency of the applied signal, the particles can experience either positive or negative DEP forces, respectively. This indicates that there must be some crossover frequency, $f_{c o}$, where the suspended particles do not experience a net force due to DEP, or when the CM factor is equal to zero. The crossover frequency [18] is given by

$$
f_{c o}=\frac{1}{2 \pi} \sqrt{-\frac{\left(\sigma_{p}-\sigma_{m}\right)\left(\sigma_{p}+2 \sigma_{m}\right)}{\left(\varepsilon_{p}-\varepsilon_{m}\right)\left(\varepsilon_{p}+2 \varepsilon_{m}\right)}}
$$

The magnitude of the DEP force depends strongly on the geometry of the electrodes responsible for the non-uniform electric field. This dependency can be expressed through a scaling law [2]

$$
\left|F_{D E P}\right| \propto \frac{V^{2}}{L_{e}^{2}}
$$

where $V$ is the applied voltage, and $L_{e}$ is the characteristic length of the electrodes. This is similar to the relationship shown in equation 10 .

One study, by Hsieh et al. [19] used a movable electrode to apply a DEP force to suspended particles. The electrode used in their experiment was a piece of pencil lead, which was etched in a solution of either sulfuric acid and potassium dichromate, or 
sodium hydroxide. In either case, a $60 \mathrm{~Hz} \mathrm{AC} \mathrm{signal} \mathrm{applied} \mathrm{to} \mathrm{the} \mathrm{electrolyte} \mathrm{and} \mathrm{the}$ graphite. The original pencil lead diameter was measured to be $500 \mu \mathrm{m}$, and was etched to a sharp point with a diameter of approximately $10 \mu \mathrm{m}[19]$.

The experiments performed by Hsieh et al. [19] were conducted by placing the pencil lead electrode into a reservoir of solution, approximately $500 \mu \mathrm{m}$ from the opposite electrode, which was an indium tin oxide (ITO) coated microscope slide. The conductivity of the solution was varied, and included conductivities of $1.0 \mathrm{E}-4,1.0 \mathrm{E}-3$, 5.0E-3, and $3.0 \mathrm{E}-2 \mathrm{~S} / \mathrm{m}$ for solutions containing latex micro-particles with diameters of 14.5 and $10.9 \mu \mathrm{m}$. For the solutions containing whole human blood samples, Hsieh et al. used solutions with conductivities of $1.0 \mathrm{E}-4,5.0 \mathrm{E}-4,3.0 \mathrm{E}-3$, and $1.5 \mathrm{E}-2 \mathrm{~S} / \mathrm{m}$. The applied voltage of the AC signal used in their experiments was $30 \mathrm{~V}_{\mathrm{rms}}$, and the frequency was varied from $20 \mathrm{~Hz}$ to $2 \mathrm{MHz}$.

In the experiments containing latex micro-particles, Hsieh et al. [19] were able to successfully demonstrate pDEP and nDEP. In their lowest conductivity solution, 1.0 E-4 $\mathrm{S} / \mathrm{m}$, their experiment showed pDEP for all applied frequencies. In all the other solutions, they were able to display both $\mathrm{pDEP}$ at lower frequencies and $\mathrm{nDEP}$ at higher frequencies. This result agreed with the established theory, as the CM factor is frequency dependent.

In the experiments containing the human blood cells, Hsieh et al. [19] was able to show $\mathrm{pDEP}$ and nDEP for each solution with a different conductivity. The nDEP was dominant at low frequencies, and the pDEP force was dominant at the higher frequencies for the experiments. At an applied frequency of $20 \mathrm{~Hz}$, however, no DEP force was 
observed for any solution of any conductivity. This was most likely due to the electrokinetic fluid flow is capable of dominating at low frequencies [9].

Hsieh et al. [19] were able to show both $\mathrm{pDEP}$ and nDEP forces acted on different particles suspended in solutions with different conductivities. Their experiments would be impractical for most researchers due to the high voltage requirement, approximately $30 \mathrm{~V}_{\text {rms. }}$. The applied voltage could very easily be lowered to achieve similar DEP force magnitudes by adjusting two parameters- the diameter of the graphite electrode, and/or the distance between the graphite electrode and the ITO microscope slide. Equation 10 quantifies the importance of the geometry of the electrodes in the producing a gradient in the electric field, while the length between the electrodes controls the strength of the electric field. The studies by Hsieh et al. [19] proved that inexpensive electrodes could be used in DEP capture and repulsion, but the applied voltage in their experiment would produce "excess electrokinetic forces," and would be unsuitable for smaller, sub-micron particles because the electrokinetic forces would completely dominate the system.

Another research study of particular interest was performed by Brown et al. [63]. In this study, Brown et al. created a tri-axial probe to be used for DEP studies. The electrode was a modified version of a commercial AFM probe. The core of the electrode was conductive, and there were alternating layers of a dielectric material and conductive material. A planar electrode surface with a total diameter of $7 \mu \mathrm{m}$ was exposed in order to conduct the experiments (Figure 2). 


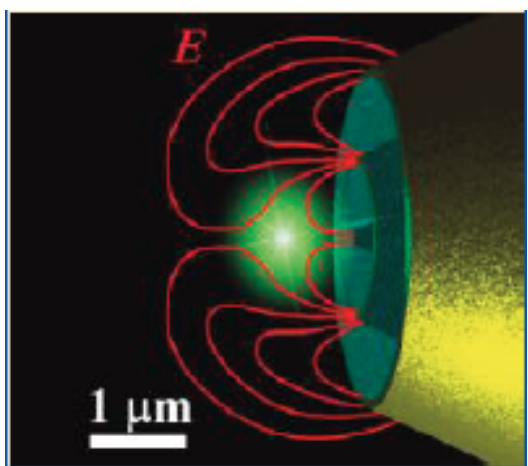

FIGURE 2 - Tri-Axial AFM Probe [63]

These experiments were done to illustrate particle trapping by nDEP [63]. The purpose of the study was to determine if "pick and place" nano-assembly was possible using DEP. The experiments successfully proved that these tri-axial AFM electrodes could capture, hold, and transport polystyrene particles with diameters of approximately $100 \mathrm{~nm}$. Their simulations showed that particles as small as $5 \mathrm{~nm}$ could be captured with the same planar configuration, but the tri-axial electrode diameter would need to be approximately $0.6 \mu \mathrm{m}$.

The tri-axial AFM configuration was chosen because it was capable of trapping particles with nDEP forces. This is due to the electrically conductive core in the AFM. An electrode with an insulating core would have a much harder time trapping particles with nDEP forces due to the generated electric field gradient. The same is true for coaxial electrodes. The pDEP force generated by coaxial electrodes can cause multiple particles to stick to both themselves and the electrode [63, 67], which is obviously not desirable for pick and place nano-assembly where individual particle capture is required.

In the experiments, Brown et al. [63] applied a $10 \mathrm{~V}_{\mathrm{pp}} \mathrm{AC}$ signal to the middle conductive layer, while grounding the inner and outermost conductive layers. The force due to pDEP was dominant at a frequency of $100 \mathrm{kHz}$, and the force due to $\mathrm{nDEP}$ was dominant at a frequency of $5 \mathrm{MHz}$. 
In the studies done by Brown et al. [63] the experiments were done with only polystyrene nano-particles suspended in a solution of 1:1 v/v deionized (DI) water and glycerol. Their experiments were able to show particle capture by nDEP forces, but equations 9,11 , and $14-18$ show that the particle capture is highly dependent on the conductivity of the particle, as well as the suspending medium. These experiments would be expected to behave in accordance with theory when a conductive medium is used. Theory predicts that the required frequency for $\mathrm{nDEP}$ forces to dominate the electrokinetic fluid flow would increase in a more conductive medium.

The method of fabricating the nano-probes, discussed in detail in Section II, is a novel one. A study by Park et al. [68] used a similar fabrication technique to create a carbon nanotube (CNT) tip on the end of an AFM probe tip. pDEP forces were used to align and attach the CNT to the AFM probe tip. A previous study by Lee et al. [69] used a conductive surface on the AFM probe to apply a pDEP force. Lee et al. [69] was able to determine that in order to attach the CNT to the tip of the AFM probe, the applied AC signal required an amplitude of greater than $5 \mathrm{~V}$ and frequency of greater than $5 \mathrm{MHz}$. Their research reasoned that the force holding the CNT to the AFM probe was primarily due to van der Waals forces.

The CNT nano-probes created by Lee et al. and Park et al. [68, 69] were used to increase the resolution of an AFM topography scan. While these CNT nano-probes have very small diameters, they would not be useful to perform DEP force studies due to the electrical resistance in the CNT. Lee et al. [69] noted that currents of $10-20 \mu \mathrm{A}$ cause the CNT to "burn and shorten." 
The classical form of the DEP force, given by equation 9, is a very good approximation of the true DEP force acting on a particle. However, this equation assumes that the suspended particles are homogenous and spherical, which is not the case for sub-cellular entities. There are many other methods of calculating the DEP force, for non-spherical, heterogeneous, and shelled particles. Equations 20 - 24 detail the calculations involved in determining the time dependent DEP force, $F_{D E P}$

$$
F_{D E P}(t)=(m(t) \cdot \nabla) E(t)
$$

where $m(t)$ is the dipole moment, and $E(t)$ is the electric field. $E(t)$ is a sinusoidal function in time, as shown by

$$
E(t)=E_{x}(t) \hat{i}+E_{y}(t) \hat{j}+E_{z}(t) \hat{k}
$$

where $E_{x}, E_{y}$, and $E_{z}$ are the electric field components along the x, y, and z-axes, respectively, and $\hat{\imath}, \hat{\jmath}, \hat{k}$ are unit vectors. The electric field components are given by

$$
E_{i}(t)=E_{i 0}(x, y, z) \cos \left(\omega t+\phi_{i}\right)
$$

where $E_{i 0}$ is the magnitude of the electric field and $\phi_{i}$ is the phase lag. The subscript $i$ indicates the direction on the Cartesian coordinate system. The total dipole moment is given by

$$
m(t)=m_{x}(t) \hat{i}+m_{y}(t) \hat{j}+m_{z}(t) \hat{k}
$$

where $m_{i}$ is the dipole moment in the $\mathrm{x}, \mathrm{y}$, and $\mathrm{z}$ directions, respectively. The dipole moment is given by

$$
m_{i}(t)=2 \Gamma \varepsilon_{m}\left(\operatorname{Re}[\mathrm{CM}] \cos \left(\omega t+\phi_{i}\right)-\operatorname{Im}[\mathrm{CM}] \sin \left(\omega t+\phi_{i}\right)\right) E_{i 0}
$$

where $\Gamma$ is the geometric factor of the particles, and $\operatorname{Re}[\mathrm{CM}]$ and $\operatorname{Im}[\mathrm{CM}]$ are the real and imaginary parts of the Clausius-Mossotti (CM) factor. The geometric factor for spherical and cylindrical particles are given by 


$$
\begin{aligned}
& \Gamma_{\text {spherical }}=2 \pi r_{p}^{3} \\
& \Gamma_{\text {cylindrical }}=\frac{\pi r_{p}^{2} l}{2}
\end{aligned}
$$

where $r_{p}$ is the radius of the particle, and $l$ is the length of the cylindrical rod [42].

Another interesting method of calculating the DEP force involves using the Hessian matrix [60]. This method is useful when ellipsoid particles are in suspended in solution. The induced dipole in an ellipsoid particle is not necessarily parallel to the electric field. In fact, unless an axis of the ellipsoid is aligned with the electric field, the induced dipole cannot be assumed to be parallel to the electric field [60]. Winter et al. presented equations 27 - 31 to account for the DEP force on dipoles that are not parallel to the electric field [60].

$$
F_{D E P}=-H(\phi) \psi
$$

where $H(\phi)$ is the Hessian matrix of the electric potential, $\phi$, and $\psi$ is the force on the dipole. The Hessian matrix of the electric potential is given by

$$
-H(\phi)_{i, j}=\frac{\partial^{2} \phi}{\partial x_{i} \partial x_{j}}
$$

where $x_{i}$ and $x_{j}$ are the directions of the potential. The previous two equations can be combined to be expressed as

$$
-H(\phi) \psi=\left[\begin{array}{lll}
\frac{\partial}{\partial x}\left(\frac{\partial \phi}{\partial x}\right) & \frac{\partial}{\partial y}\left(\frac{\partial \phi}{\partial x}\right) & \frac{\partial}{\partial z}\left(\frac{\partial \phi}{\partial x}\right) \\
\frac{\partial}{\partial x}\left(\frac{\partial \phi}{\partial y}\right) & \frac{\partial}{\partial y}\left(\frac{\partial \phi}{\partial y}\right) & \frac{\partial}{\partial z}\left(\frac{\partial \phi}{\partial y}\right) \\
\frac{\partial}{\partial x}\left(\frac{\partial \phi}{\partial z}\right) & \frac{\partial}{\partial y}\left(\frac{\partial \phi}{\partial z}\right) & \frac{\partial}{\partial z}\left(\frac{\partial \phi}{\partial z}\right)
\end{array}\right]\left[\begin{array}{l}
\psi_{x} \\
\psi_{y} \\
\psi_{z}
\end{array}\right]
$$

which, when multiplied out, gives 


$$
-H(\phi) \psi=\left[\begin{array}{l}
\psi_{x} \frac{\partial}{\partial x}\left(\frac{\partial \phi}{\partial x}\right)+\psi_{y} \frac{\partial}{\partial y}\left(\frac{\partial \phi}{\partial x}\right)+\psi_{z} \frac{\partial}{\partial z}\left(\frac{\partial \phi}{\partial x}\right) \\
\psi_{x} \frac{\partial}{\partial x}\left(\frac{\partial \phi}{\partial y}\right)+\psi_{y} \frac{\partial}{\partial y}\left(\frac{\partial \phi}{\partial y}\right)+\psi_{z} \frac{\partial}{\partial z}\left(\frac{\partial \phi}{\partial y}\right) \\
\psi_{x} \frac{\partial}{\partial x}\left(\frac{\partial \phi}{\partial z}\right)+\psi_{y} \frac{\partial}{\partial y}\left(\frac{\partial \phi}{\partial z}\right)+\psi_{z} \frac{\partial}{\partial z}\left(\frac{\partial \phi}{\partial z}\right)
\end{array}\right]
$$

The force acting on the dipole, $\psi_{i}$, is expressed by

$$
\psi_{i}=v_{p} \varepsilon_{0}\left(\varepsilon_{p}-\varepsilon_{m}\right) E_{i}^{*}
$$

were $v_{p}$ is the volume of the particle, $\varepsilon_{0}$ is the permittivity in a vacuum, and $E_{i}^{*}$ is the internal field in a particle [60].

\section{Electrohydrodynamic Forces}

a. Heating Effects. When an electric field is applied to a colloidal suspension, the suspended particles experience forces due to the relative motion of the medium. The applied forces due to the heating of the fluid are deemed electrothermal forces. It is important to consider these electrothermal forces because of their effects on the system. Electrothermal fluid forces can be of benefit or of detriment to the system. For example, electrokinetic or electrothermal fluid flow can facilitate the transport of particles. On the other hand, large temperature differences can cause bubble formation [70], which can damage the system. Studies by Ramos, et al. [71] have shown that the differential temperature can be expressed as

$$
\frac{\Delta T}{T} \approx \frac{1}{2 \omega t_{d i f f}}
$$

where $t_{\text {diff }}$ is the time to reach the equilibrium temperature. The value of $t_{\text {diff }}$ can be expressed as

$$
t_{\text {diff }}=\frac{\rho_{m} c l^{2}}{k_{m}}
$$


where $\rho_{m}$ is the density of the medium, $c_{p}$ is the specific heat of the medium, $l$ is the characteristic length of the system, and $k_{m}$ is the thermal conductivity of the medium [71]. The differential temperature is negligible for AC signals with frequencies greater than 1 $\mathrm{kHz}$ [71]. In this work, the characteristic length of the system is typically less than 40 $\mu \mathrm{m}$, and the medium is water. The density of the medium is $1000 \mathrm{~kg} \mathrm{~m}^{-3}$, the specific heat is $4.18 \mathrm{~kJ} \mathrm{~kg}^{-1} \mathrm{~K}^{-1}$, and the thermal conductivity is $0.6 \mathrm{~J} \mathrm{~m}^{-1} \mathrm{~s}^{-1} \mathrm{~K}^{-1}$. These values give $t_{\text {diff }}$ a value of $11.1 \mathrm{~ms}$. $11.1 \mathrm{~ms}$ is a fairly typical time value for the temperature to reach steady state $[70,71]$. However, the time for thermal diffusion is much slower, on the order of seconds [70].

An estimate of the temperature rise due to the applied electric field is given by

$$
\Delta T=\frac{\sigma_{m} V_{r m s}^{2}}{k}
$$

which is accurate to an order-of-magnitude [71]. Equation 34 shows that the temperature rise is also linearly proportional to media conductivity and the square of the applied potential. Equation 10 shows the electric field is also linearly proportional to the square of $V_{r m s}$. This relationship shows that the temperature difference is decreased as voltage is decreased, but so is the electric field strength. A lower local temperature difference reduces the local convective heat transfer away from the electrode $[9,71]$ which, in turn, reduces the fluid velocity in the system.

One of the major heating sources is a form of resistance heating called Joule heating. Joule heating occurs in the liquid when an electric field is applied through a fluid. The elevated temperature can be great enough to denature live cells [47], which could be problematic if living cells are to be probed. Joule heating may cause intense local temperature gradients. These temperature gradients cause a density change in the fluid, 
which forces the warmed liquid away from the electrodes. The low-density fluid is replaced with a fluid of higher density. This process was named the "thermal end effects" of electroosmosis by Xuan, et al. [47]. These "thermal end effects" and Joule heating can be beneficial when studying microchannel flow because the flow is considered to be very nearly true plug flow. This allows the fluid to be pumped through the microchannel by means of an electric field. However, for the studies done in this thesis, Joule heating is considered to act to the detriment of the system, which is typical for most experiments [72]. The Joule heating power is expressed by [72]

$$
P=G V^{2}=\frac{\pi \sigma_{m} d_{p}^{2} \nu^{2}}{4 L}=\frac{\pi \sigma_{m} d_{p}^{2} E^{2} L}{4}
$$

where $G$ is the conductance of the electrolyte, $V$ is the separation voltage, $L$ is the length between electrodes, and $E$ is the electrical field strength. The conductance of the medium in equation 35 is assumed to be constant. This is not necessarily a valid assumption. Many researchers have noted the existence of a positive feedback loop between conductance and temperature [72]. This relationship can be expressed by

$$
\sigma_{m, \text { mean }}=\sigma_{m, 298}+\gamma_{1}\left(T_{\text {mean }}-298\right)+\gamma_{2}\left(T_{\text {mean }}-298\right)^{2}
$$

where $\sigma_{m, \text { mean }}$ and $\sigma_{m, 298}$ are the average conductivity and the conductivity at $298 \mathrm{~K}$, respectively, $T_{\text {mean }}$ is the average temperature, and $\gamma_{1}$ and $\gamma_{2}$ are temperature coefficients with units of $\mathrm{K}^{-1}$ and $\mathrm{K}^{-2}$, respectively [72]. This relationship indicates that as temperature increases, so does the conductance of the fluid. Likewise, as the conductance of the fluid increases, so does the temperature when an electric field is applied. The permittivity of water, measured in $\mathrm{C} \mathrm{V}^{-1} \mathrm{~m}^{-1}$, which is the fluid medium in this work, also is temperature dependent. The permittivity of water, $\varepsilon_{\mathrm{m}}$, can be expressed as [72] 


$$
\varepsilon_{m}=2.707 * 10^{-9} e^{-T / 219}
$$

Another property that plays an important role in the dispersion of particles is called the zeta potential, $\zeta$. The zeta potential is a property of particles suspended in a medium. The zeta potential shows the strength of the electrical double layer (EDL) between particles and describes the potential for particles to flocculate or coagulate [73]. The zeta potential also helps describe how the particles move in the system. A high zeta potential leads to a more uniform dispersion, while a lower zeta potential leads to coagulation of the particulate [73].

The conductivity of particles is also inversely proportional to the radius of the particles. The conductivity, $\sigma_{p}$, can be expressed as

$$
\sigma_{p}=\sigma_{b}+\frac{2 K_{s}}{r_{p}}
$$

where $\sigma_{p}$ is the bulk conductivity of the particles, and $K_{s}$ is the surface conductance. [74]. For polystyrene particles, the surface conductance is up to $1.2 \mathrm{nS}$ [75] and the bulk conductivity is zero.

$\mathrm{AC}$ electro-osmotic flow (ACEOF) is the steady motion of the liquid [9] when the fluid is exposed to an electric field. The fluid velocity has been shown to be a function of the applied potential and frequency $[9,76]$. In most cases, the velocity due to ACEOF is very small, and is often negligible when compared to other electrothermal forces.

b. Electrothermal Hydrodynamics. Forces that induce fluid flow due to temperature gradients in the dielectric properties of the media are called electrothermal forces. These forces per unit volume can be expressed by

$$
\boldsymbol{F}_{e}=\rho_{q} \boldsymbol{E}-\frac{1}{2} \boldsymbol{E}^{2} \nabla \varepsilon_{m}+\frac{1}{2} \nabla\left(\rho_{m} \frac{\partial \varepsilon_{m}}{\partial \rho_{m}} \boldsymbol{E}^{2}\right)
$$


where $\rho_{q}$ is the volume charge density of the particles. The last term in the equation, the electrostriction force, is zero when the medium is an incompressible fluid [71]. In an incompressible fluid, the permittivity does not change with respect to the density of the medium. This causes the partial derivative term to become a scalar quantity, and the gradient of a scalar is zero [71]. The first term in equation 39 is known as the Coulomb force, and the second is known as the dielectric force. Both of these forces require gradients in the conductivity and permittivity [9]. Castellanos et al. [9] developed a scaling law for the time average electric force, $\left\langle F_{e}\right\rangle$, from an applied AC signal. This scaling law can be expressed by

$$
\left\langle F_{e}\right\rangle=\frac{1}{2} \operatorname{Re}\left[\rho_{q} \boldsymbol{E}^{*}\right]-\frac{1}{4} \boldsymbol{E} \cdot \boldsymbol{E}^{*} \nabla \varepsilon
$$

where $\boldsymbol{E}^{*}$ is the complex conjugate of the electric field. The charge density can be expressed as

$$
\rho_{q}=\frac{\sigma \nabla \varepsilon-\varepsilon \nabla \sigma}{\sigma+\mathrm{i} \omega \varepsilon} \cdot \boldsymbol{E}_{0}
$$

More commonly, the charge density is expressed as [53]

$$
\rho_{q}=\frac{\sigma \varepsilon(\alpha-\beta)}{\sigma+\mathrm{i} \omega \varepsilon} \nabla T \cdot \boldsymbol{E}_{0}
$$

where $\alpha$ and $\beta$ are expressed as

$$
\begin{aligned}
& \alpha=\frac{1}{\varepsilon} \frac{\partial \varepsilon}{\partial T} \\
& \beta=\frac{1}{\sigma} \frac{\partial \sigma}{\partial T}
\end{aligned}
$$

For an aqueous $\mathrm{KCl}$ solution, which was used in this work, the coefficients $\alpha$ and $\beta$ have values of approximately $-0.4 \% \mathrm{~K}^{-1}$ and $2 \% \mathrm{~K}^{-1}$, respectively [53]. The result of combining equations 40 - 44 can be expressed as

$$
\left\langle F_{e}\right\rangle=\frac{1}{2} \operatorname{Re}\left[\left(\frac{\sigma \nabla \varepsilon-\varepsilon \nabla \sigma}{\sigma+\mathrm{i} \omega \varepsilon} \cdot \boldsymbol{E}_{0}\right) \boldsymbol{E}_{0}^{*}\right]-\frac{1}{4} \boldsymbol{E}_{0} \cdot \boldsymbol{E}_{0}^{*} * \nabla \varepsilon
$$




$$
\left\langle F_{e}\right\rangle=\frac{1}{2} \operatorname{Re}\left[\frac{\sigma \varepsilon(\alpha-\beta)}{\sigma+i \omega \varepsilon}\left(\nabla T \cdot \boldsymbol{E}_{0}\right) \boldsymbol{E}_{0}^{*}-\frac{1}{2} \epsilon \alpha\left|\boldsymbol{E}_{0}\right|^{2} \nabla T\right]
$$

Equation 45a shows that the electrical force is dependent on the frequency of the applied AC signal. If $\omega$ is much larger than $\frac{\sigma}{\varepsilon}$, the dielectric force dominates. If $\frac{\sigma}{\varepsilon}$ is much larger than $\omega$, the Coulomb force dominates.

\section{Total Particle Motion - An Overview}

DEP forces must dominate Brownian motion to be observed in the system [18]. The best method of determining which force is dominant is by comparing the calculated velocity due to each force. The velocity of the particles due to Brownian motion is simply the time derivative of equation 7 , and is expressed by

$$
\frac{d x_{r m s}}{d t}=\frac{\sqrt{2} D}{2 \sqrt{D t}}
$$

and the velocity due to the DEP force, $v_{D E P}$, is given by

$$
v_{D E P}=\frac{r_{p}^{2} \varepsilon_{m}}{6 \mu_{m}} \operatorname{Re}[\mathrm{CM}] \nabla|E|^{2}
$$

The effects of fluid flow can also influence particle trapping. In some instances, fluid flow benefits DEP capture by circulating the solution and bringing additional particles near the electrodes. However, in other cases the fluid flow is very problematic. This work utilizes a cantilevered micro-electrode, or nano-probe, which can be damaged easily. The electrothermal fluid flow is greatest at the area with the highest temperature gradient, and fluid velocity scales with $V^{4}[71]$. The moment created by the forces acting at the end of the nano-probe can be destructive.

The last major force acting on the system is the gravitational force. This force, also known as the settling force, decreases with the volume of the particle, as shown in 
equation 1 , and is sometimes negated due to buoyancy forces due to the thermal gradients in the system.

\section{Nano-Structure Self-Assembly}

Equation 19 from the Section I part C demonstrates the requirement for small electrodes to trap nanometer particles. A smaller electrode requires less voltage to produce a gradient in the electric field to achieve DEP particle capture. Further, using less voltage is desirable since Joule heating and fluid flow increase non-linearly with applied voltage ( $V^{2}$ and $V^{4}$, respectively) [71]. A technique has been developed that produces nano-structures using a reaction between gallium and silver $[4,77]$, and the work herein has used these structures to generate large field gradients.

Liquid gallium naturally alloys with a variety of materials at room temperature, including silver, $\mathrm{Ag}$; gold, $\mathrm{Au}$; platinum, $\mathrm{Pt}$; aluminum, $\mathrm{Al}$; and copper, $\mathrm{Cu}$ [4]. The alloys create an assortment of nano-structures with different stoichiometric ratios of the cation to anion. For example, the gallium reacts with gold to produce $\mathrm{AuGa}_{2}$, which forms a crystalline structure, the gallium reacts with platinum to create $\mathrm{Ga}_{6} \mathrm{Pt}$, which forms crystalline plate structures. Finally, the gallium reacts with silver to create silver gallium, which forms crystalline rods. The spontaneous formation of the nano-structures has been termed "self-assembly" and has been widely studied for the materials and alloys listed above $[4,78]$.

The self-assembly of nano-structures is a fascinating occurrence. A general definition of self-assembly is: "the autonomous organization of the components into patterns or structures without human intervention [79]." Many researchers have noted 
that self-assembly is challenging to achieve as well as control $[24,40,41,79]$, but the results can be of great interest. Self-assembly is considered to be difficult due to basic thermodynamics. The system would prefer to be in a state of disarray rather than an ordered state [79]. The thermodynamic barrier must be overcome in order for selfassembly to be achieved. The difference between the free energies of the initial and final state is the driving force that that creates the self-assembled structures [4].

The silver gallium alloy is of particular interest because of the nano-structure that is created, a virtually cylindrical crystalline rod. The initial research done by Yazdanpanah et al. $[4,77]$ was done by placing gallium on thin films of metals, silver in this case. The reaction was carefully studied and characterized using a variety of techniques such as scanning electron microscopy, transmission electron microscopy, atomic force microscopy, and energy dispersive microscopy. X-ray diffraction patterns and the transmission electron microscopy studies show that the silver gallium has a hexagonal close packed (HCP) crystalline structure with between 8 and 16 facets that form a nearly circular cross section $[4,77,80]$.

One of the most interesting characteristics of the silver gallium is its ability to selfassemble into nano-structures with high aspect ratios. In the research done by Yazdanpanah et al. [4, 77], these nano-structures self-assembled on thin films of silver. The nano-structures were also capable of self-assembling on sharpened tips of commercial AFM probes. When the nano-structures formed on the thin silver film, the nano-structures self-assembled in highly anisotropic directions. However, single nanostructures were created when the nano-structures self-assembled on the end of the AFM probes. The directionality of the nano-structures was able to be controlled when on the 
AFM probes because the nano-structures tend to self-assemble tangentially to a tapered surface. The diameter of the assembled structures was typically between 100 and 500 $\mathrm{nm}$, but has been documented as small as $65 \mathrm{~nm}$ or as large as several microns in diameter. The nano-structures have had lengths documented up to $33 \mu \mathrm{m}[4,77]$. For the duration of this thesis, the nano-structures will be referred to as nano-probes and the selfassembly processes that are responsible for creating the nano-probes will be referred to as the growth of the nano-probe. A more detailed methodology for growing nano-probes can be found in Section II, part A of this thesis.

The silver gallium nano-probes have been used in a variety of applications. The nano-probes that were grown on atomic force microscopy (AFM) probes were able to perform AFM topography, in both tapping and non-contact modes; and nano-probes on AFM probes were used in voltage lithography $[4,77]$. Additional studies by Biedermann et al. and Dobrokhotov, et al. [80, 81] used the self-assembled silver gallium nanostructures in force and mass sensing applications. 


\section{FABRICATION AND EXPERIMENTS}

\section{A. Nano-Probe Fabrication}

A detailed procedure must be followed in order to create the silver gallium nanoprobes used in this work. The nano-probe fabrication consists of five major steps: (1) the creation of a sharp tip on a capillary tube, (2) sputter coating the capillary tube with a chromium (Cr) and silver layer, (3) the preparation of a gallium sample, (4) the growth of the nano-probe onto the sharp tip of the capillary tube, and (5) the sputter coating of the capillary tube and nano-probe with a thin layer of chromium and gold. The following section goes into more detail about the instrumentation and equipment used to create the nano-probes.

In order to begin the nano-probe fabrication process, a capillary tube must be drawn out, or pulled, to a sharp point. The borosilicate capillary tubes used in this work were purchased from Sutter Instruments with an outside diameter (OD) of $1.0 \mathrm{~mm}$ and an inside diameter (ID) of $0.75 \mathrm{~mm}$. The capillary tubes were heated by a $\mathrm{CO}_{2}$ laser and pulled apart, which results in a very sharp tip. The instrument used to create sharp probes from the borosilicate capillary tubes was the Sutter Instruments Micro-Pipette Puller, shown in Figure 3. 


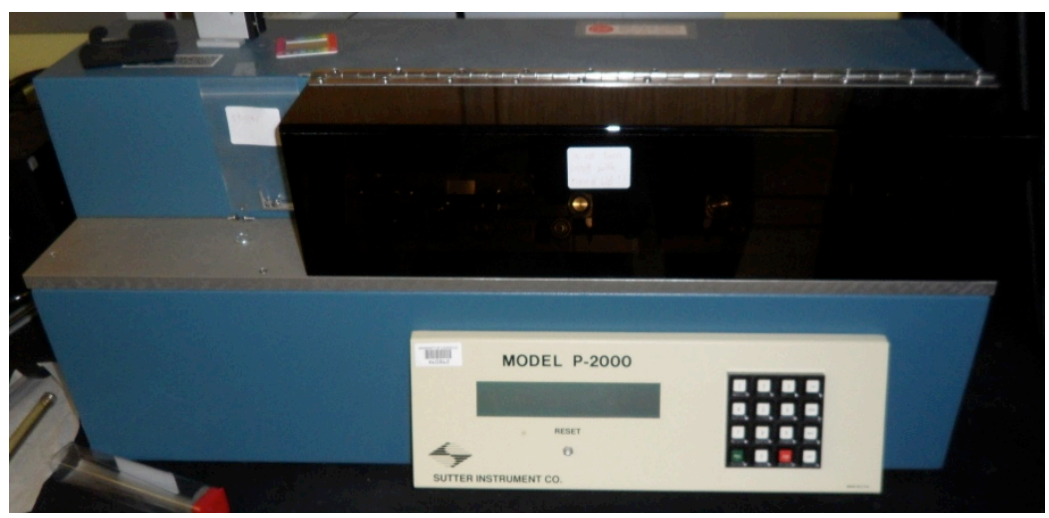

FIGURE 3 - Sutter Instruments Micro-Pipette Puller

The tip profile is dependent on laser intensity, laser scanning profile, the time delay from when the laser is switched off until the mechanical pulling begins, and the applied mechanical pulling force. These settings are referred to as Heat, Filament (Fil), Velocity (Vel), Delay (Del), and Pull, respectively. The 'Heat' setting specifies the output power of the laser. The Filament specifies the longitudinal scanning pattern of the laser. The 'Velocity' controls the speed at which the capillary tube is initially pulled apart. The 'Pull' parameter controls the force applied to the capillary tube. The 'Delay' controls the time between when the heat is off and the pull begins [82]. A sharp probe will be created if the correct parameters used. A sharp probe helps ensure that the nano-probe will be pulled with the desired orientation [4]. The recipe used to create sharp probes, which was obtained from the Sutter Instruments User Manual, is listed in Table I. A typical sharp probe is shown in Figure 4.

TABLE I

LASER SETTINGS FOR SHARP PROBE CREATION

\begin{tabular}{|c|c|c|c|c|}
\hline Heat & Fil & Vel & Del & Pull \\
\hline 350 & 4 & 50 & 225 & 150 \\
\hline
\end{tabular}




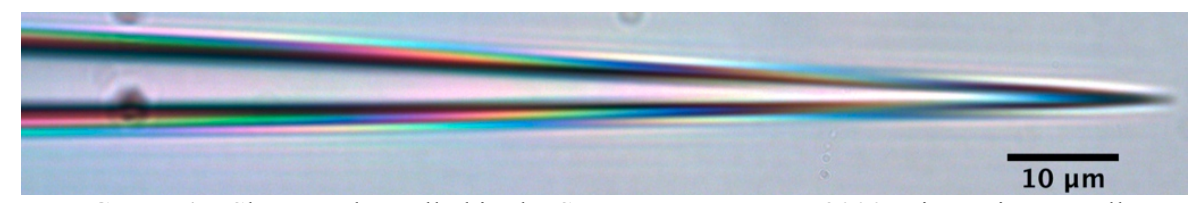

FIGURE 4 - Sharp Probe Pulled in the Sutter Instruments P-2000 Micro-Pipette Puller

After sharp probes have been created, a chromium and silver layer was deposited on the probes via sputtering in the PVD 75, manufactured by the Kurt J. Lesker Company and shown in Figure 5.

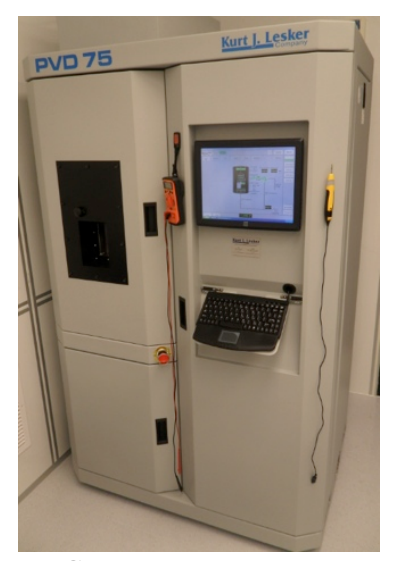

FIGURE 5 - KJL PVD 75

Sputtering is a process in which very thin films of material can be applied to a substrate. The base material, called a sputtering target, is placed in the sputtering chamber and is bombarded with an inert gas, typically a noble gas such as argon. The gas molecules collide elastically with the sputtering target, which expels the material from the sputtering target into the process chamber. Prior to sputtering, the process chamber is pumped to a high vacuum in order to reduce the energy robbing collisions between the argon gas and air. The PVD 75 utilizes a series of strong permanent magnets to help increase the ionization of argon. Increased ionization prevents some of the collisions between the argon gas molecules [83].

In the second fabrication step, a thin layer of chromium (99.95\% purity) and silver (99.99\% purity) was sputtered onto the glass capillary tube. Chromium served as an 
adhesion promoter for the silver on the glass capillary tube. The process chamber in the sputtering machine was pumped down to a base pressure of $5 \mathrm{E}-5 \mathrm{mTorr}$ and the capacitance manometer (capman) pressure for the argon gas was set at 5 mTorr. The power to the sputtering targets was set at $300 \mathrm{~W}$ DC. A plasma was generated using this power setting. The plasma was responsible for the deposition of the metals from the sputtering target onto the substrate.

A native metal oxide layer will form on the surface of the target if the sputtering targets have been left in ambient conditions,. An oxide layer on the chromium target could reduce the adhesion between the glass and the silver; an oxide layer on the silver would prevent the self-assembly of the nano-probes. To prevent any native oxide from being applied to the glass probes, a technique called 'pre-sputtering' was used. Power was supplied to each sputtering target for one to three minutes before the glass probes were exposed to the plasma. Once the glass capillary tubes were exposed in the process chamber, chromium was sputtered for 30 seconds and silver was sputtered 4 minutes onto the probes. This applied a layer of chromium and silver approximately $300 \mathrm{~nm}$ thick uniformly over the probe.

The third step in nano-probe fabrication was the preparation of the gallium sample. A fraction of a frozen gallium pellet was melted and thinly spread across a cleaned silicon ( $\mathrm{Si}$ ) substrate. Elemental gallium is a highly reactive metal in atmospheric conditions [4], and when gallium is in the liquid phase, the outer layer oxidizes almost immediately to form an oxide "skin." In order to strip the oxide layer, the gallium coated silicon wafer was placed in a $0.1 \mathrm{M}$ hydrochloric acid $(\mathrm{HCl})$ bath at $60^{\circ} \mathrm{C}$ for approximately 60 seconds [4]. The $\mathrm{HCl}$ bath stripped the oxidation layer from the 
gallium and allowed the cohesive forces in the gallium to overcome the adhesion between the gallium and the silicon. The gallium formed microscopic spheres within seconds of being placed in the acid bath.

The most critical part of fabrication was the process of "pulling" the nano-probe, or allowing the nano-probe to self-assemble. This was done using an optical microscope with a 100X, 0.9 N.A. Mitutoyo objective with a working distance of $6.0 \mathrm{~mm}$, and viewed on a computer via a 5 MP Pixel PL-B778F camera. The optical microscope setup used is shown in Figure 6.

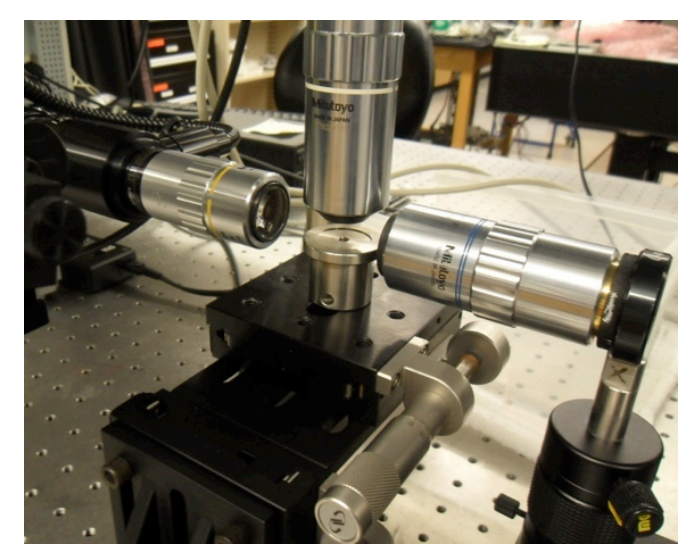

FIGURE 6 - Microscope for Viewing Nano-Probe Creation

The silver coated glass capillary tube was placed in a World Precision Instruments Piezo-Patch $^{\mathrm{TM}}$ PPM5000 nano-manipulator and guided into a gallium droplet. The manipulator consists of a controller as well as a three axis linear actuator, which are shown in Figures 7 A) and B). 


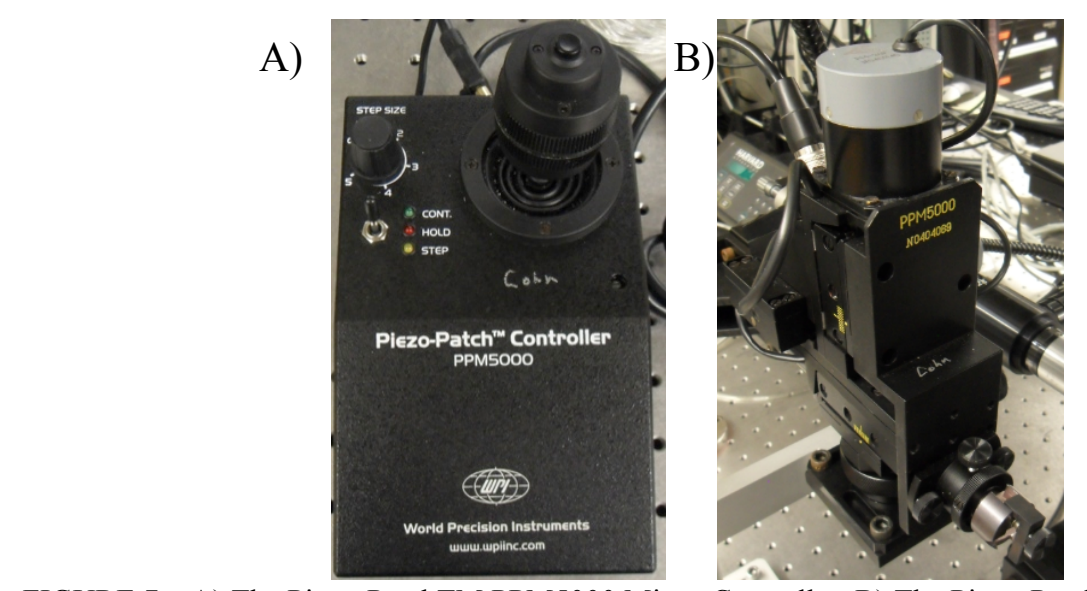

FIGURE 7 - A) The Piezo-PatchTM PPM5000 Micro-Controller. B) The Piezo-PatchTM PPM 5000 NanoManipulator

The diameter of the gallium droplet was a critical parameter. A large gallium droplet, i.e. one greater than $40-50 \mu \mathrm{m}$ in diameter, would remove the silver and chromium from the tip of the probe and could result in a bare glass probe or an incorrect stoichiometric ratio for nano-probe formation. A small gallium droplet, i.e. one smaller than $5 \mu \mathrm{m}$ in diameter, could attach itself to the end of the probe and solidify. Once an adequately sized gallium droplet was located, the silver-coated capillary tip was inserted into the droplet, then pulled back slightly to form a reverse meniscus [4]. The microelectrode was left in the gallium droplet for approximately two minutes, and then was removed from the gallium droplet at a rate of approximately $150 \mu \mathrm{m} / \mathrm{min}$. At this point, there were two possible outcomes. Either a nano-probe was present, or there was no nano-probe located on the micro-electrode. If no nano-probe was formed after the first attempt, the probe was inserted into the gallium droplet again, but for a shorter duration of time -typically one minute. The micro-electrode could be inserted into the gallium droplet multiple times until the chromium and silver coating was completely removed by the gallium, or until gallium collected on the surface of the micro-electrode, rendering it useless because the silver layer was no longer exposed. A nearly $90 \%$ success rate of 
nano-probe formation can be achieved by an experienced user. Figure 8 shows an illustration of the nano-probe pulling process.
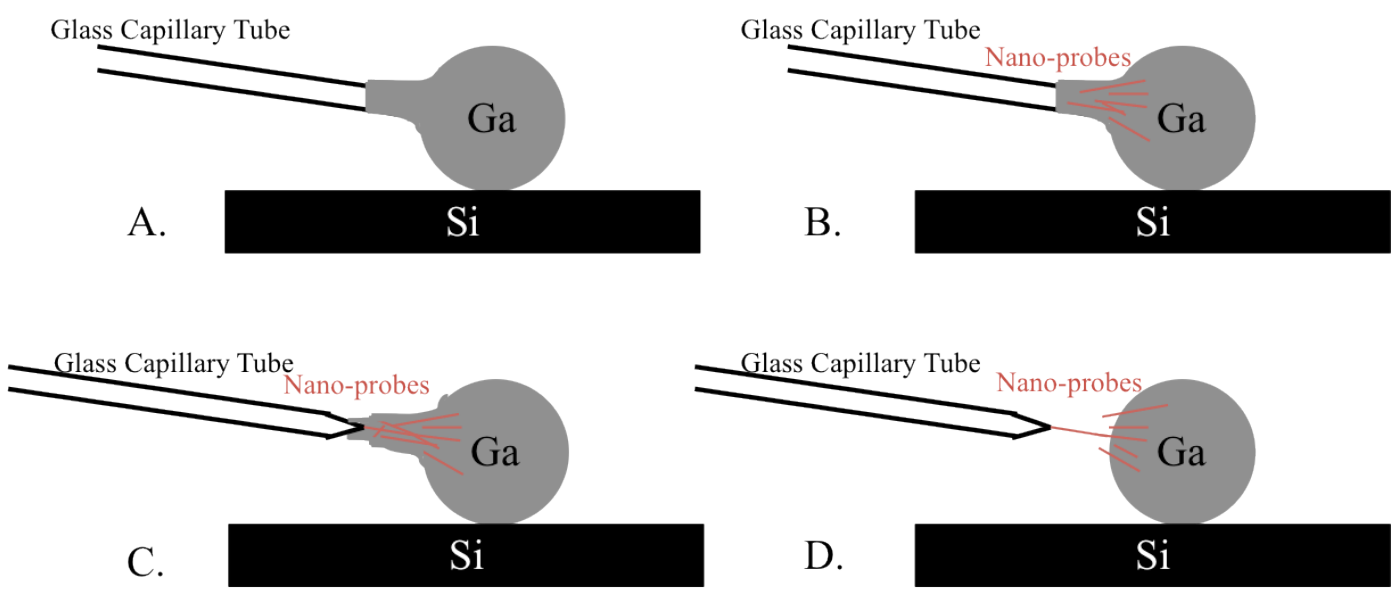

FIGURE 8 - Diagram of the Nano-Probe Pulling Process: (A) The Reverse Meniscus, (B) Nano-probe Formation, (C) Glass Capillary Tube Withdrawal, and (D) Nano-Probe Attached to Capillary Tube

Once the nano-probes were "pulled" onto the micro-electrode, the entire structure was sputter coated in gold in the PVD 75. Silver gallium oxidizes and becomes nonconductive over a period of one to two days; the gold coating allows the nano-probes to have a longer useful life since gold does not oxidize. Another benefit of sputter-coating gold was to provide a layer of conductive material from the shaft of the capillary tube to the nano-probe. As the capillary tube was retracted from the gallium droplet, the gallium removed a large quantity of conductive material from the capillary tube, which would electrically isolate the nano-probe from the conductive material higher up on the shaft; this is illustrated in Figure 9. 
A.

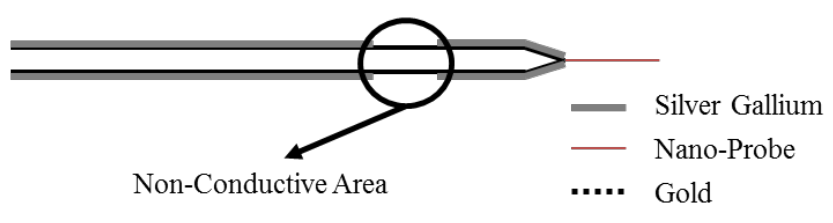

B.

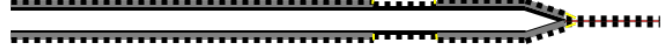

FIGURE 9 - Typical Nano-Probes. (A) Conductive Layers of Silver Separated by Glass Does Not Permit a Signal to be Sent to the Nano-Probe, (B) Conductive Layers of Silver Separated by Glass, but Coated in Gold to Permit Electron Movement

To coat the capillary tube and nano-probe in gold (99.99\% purity), the probes were placed in the PVD 75. The gold required an adhesion promoter; a thin chromium layer was sputtered prior to the gold film. The chromium was sputtered onto the device for 0.5 minutes, and the gold for 2.5 minutes. A capman pressure of $10 \mathrm{mTorr}$ of argon and a power setting of $300 \mathrm{~W}$ DC was used to apply an approximately $150 \mathrm{~nm}$ thick layer of chromium and gold uniformly over the nano-probe and micro-electrode.

\section{B. Sample Preparation}

The effects of DEP and electrokinetic forces were tested on two different types of nano-particles, polystyrene and gold. The majority of the experiments demonstrated DEP and electrokinetic forces acting on spherical polystyrene particles in suspended in solutions with various conductivity levels. The other experiments were to determine if even smaller particles could be captured using DEP. For the latter experiments, solutions of gold nano-particles suspended in DI water were used.

The experiments with polystyrene particles were prepared by putting approximately four drops of Fluoro-Max ${ }^{\mathrm{TM}}$ particles in solution with $7.5 \mathrm{~mL}$ of $99.5 \%$ aqueous media and $0.05 \%$ polysorbate 20 (Tween $20^{\circledR}$ ). Tween $20^{\circledR}$ is a detergent that was used to prevent particle agglomeration within the suspending medium. The Fluoro-Max ${ }^{\mathrm{TM}}$ particles were manufactured and shipped in a solution containing 1\% solids. Three 
different sizes of Fluoro-Max ${ }^{\mathrm{TM}}$ particles were used, with diameters of $200 \mathrm{~nm}, 500 \mathrm{~nm}$, and $1000 \mathrm{~nm}$. The Fluoro-Max ${ }^{\mathrm{TM}}$ particles were excited by light with a wavelength of $542 \mathrm{~nm}$ and emitted a wavelength of $612 \mathrm{~nm}$. These particles were illuminated by a green light source and fluoresced red.

Four drops of particles, assuming each drop was $0.02 \mathrm{~mL}$, with a concentration of $1 \%$ solids meant that there were approximately 1.53 billion of the $1000 \mathrm{~nm}$ particles, 12.2 billion of the $500 \mathrm{~nm}$ particles, and 191 billion of the $200 \mathrm{~nm}$ particles added to the 7.5 $\mathrm{mL}$ of aqueous media. The solutions used in the experiments contained approximately 204 million of the $1000 \mathrm{~nm}$ particles per $\mathrm{mL}, 1.63$ billion of the $500 \mathrm{~nm}$ particles per $\mathrm{mL}$, and 25.5 billion of the $200 \mathrm{~nm}$ particles per $\mathrm{mL}$. These particles were added to DI water or an aqueous solution of potassium chloride $(\mathrm{KCl})$. The aqueous $\mathrm{KCl}$ solution was prepared by dissolving solid $\mathrm{KCl}$ in DI water. The highest conductivity used was 2.26E-1 $\mathrm{S} / \mathrm{m}$, the lower conductivity used was $2.5 \mathrm{E}-2 \mathrm{~S} / \mathrm{m}$, and the lowest conductivity used was DI water, which has a conductivity of $5.5 \mathrm{E}-8 \mathrm{~S} / \mathrm{m}$. The conductivity of the medium was increased to simulate how the cytoplasm in a mammalian cell $\left(\sigma_{\mathrm{m}}=1 \mathrm{~S} / \mathrm{m}\right)$ might react to applied electric fields [44].

The gold nano-particles were supplied by Dr. Gobin's laboratory. The larger nanoshells, with diameters of approximately $120 \mathrm{~nm}$, were fabricated using the Stöber method. This method requires silica particles to be functionalized with an amine, aminopropyl triethoxsilane, then exposed to colloidal gold. The colloidal gold was adsorbed, and this gold was used as a nucleation site for the reduction of additional gold onto the surface [84]. The smaller gold nano-particles, with hydraulic diameters between five and fifty nanometers, were prepared by mixing a solution of gold chloride, 
$\mathrm{HAuCl}_{4} \cdot 3 \mathrm{H}_{2} \mathrm{O}$, with sodium thiosulphate, $\mathrm{Na}_{2} \mathrm{~S}_{2} \mathrm{O}_{3}$. This solution was allowed to react at room temperature for approximately one hour, after which the nano-particles had formed.

\section{Experimental Setup}

In order to generate an electric field gradient capable of producing DEP forces, the nano-probes must be placed in proximity to another electrode that is oppositely biased. In this work, the second electrode was an indium tin oxide (ITO) coated coverslip. The ITO coverslip was connected to ground, while the gold-coated nano-probe was connected to the AC signal. Section I examines why the distance between the electrodes is a very important variable in controlling the strength and gradient of the electric field. This distance between electrodes was typically less than $40 \mu \mathrm{m}$, measured manually using the Nikon Eclipse Ti microscope, shown in Figure 10, by the difference in focal distance between the upper surface of the ITO coverslip and the tip of the nano-probe.

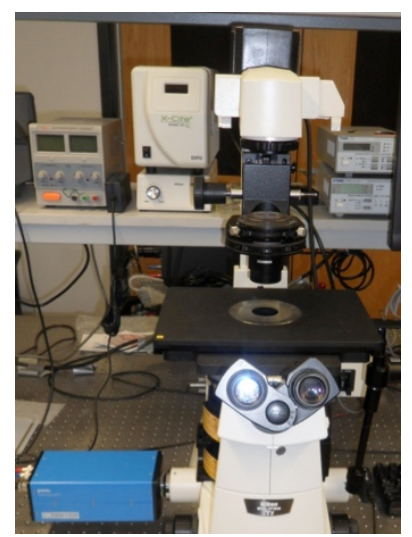

FIGURE 10 - Nikon Eclipse Ti Microscope

The experiments were set up by placing a drop of solution on the ITO coverslip, then inserting the nano-probe is into the droplet and applying an AC signal. The AC signal 
was applied to the electrodes through a wire lead soldered to a piece of copper tape. The copper tape was attached to the conductive portion of the capillary tube. Figure 11shows an illustration of the experimental set-up.

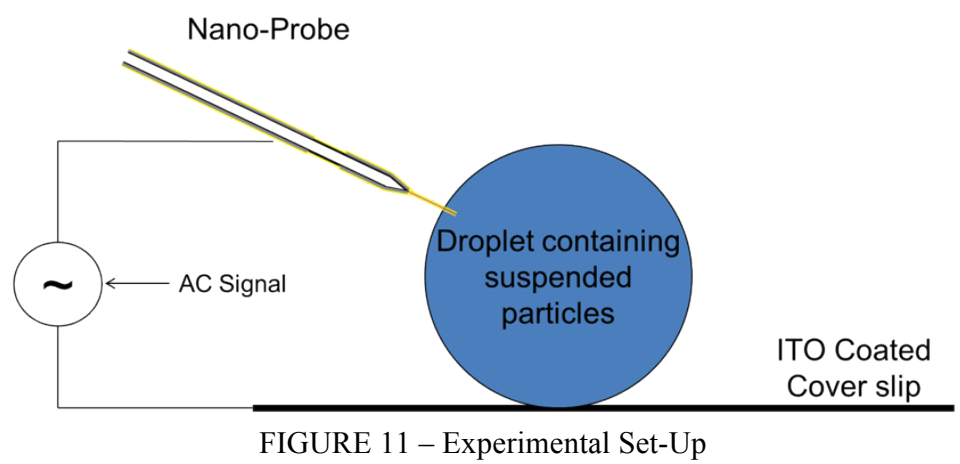

The polystyrene nano-particles were observed under the Nikon Eclipse Ti, shown in Figure 10. The gold nano-particles were observed under both the Nikon Eclipse Ti, as well as an Olympus IX71 inverted microscope, shown in Figure 12.

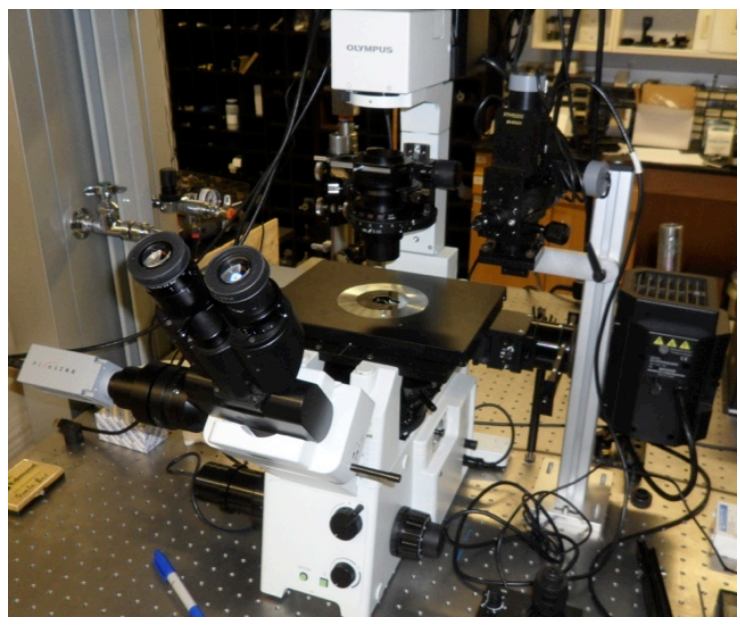

FIGURE 12 - Olympus IX71 Microscope 


\section{RESULTS AND DISCUSSION}

\section{A. Nano-Probe Fabrication Results}

Figure 13 shows an image of a nano-probe captured by the Olympus IX71 under 40X magnification. Figures 14-15 show images of nano-probes captured with the Mitutoyo 100X objective. Figure 14 also shows the gallium droplet from which the nano-probe was pulled. Figures 16-17 show scanning electron microscopy images of nano-probes that have been sputter coated with gold. The fabricated nano-probes typically had diameters $\geq 150 \mathrm{~nm}$. The length of the nano-probes varied from $<1 \mu \mathrm{m}$ up to $30 \mu \mathrm{m}$. Nano-probes with diameters of approximately $250 \mathrm{~nm}$ and lengths of 10-12 $\mu \mathrm{m}$ were manufactured most frequently.

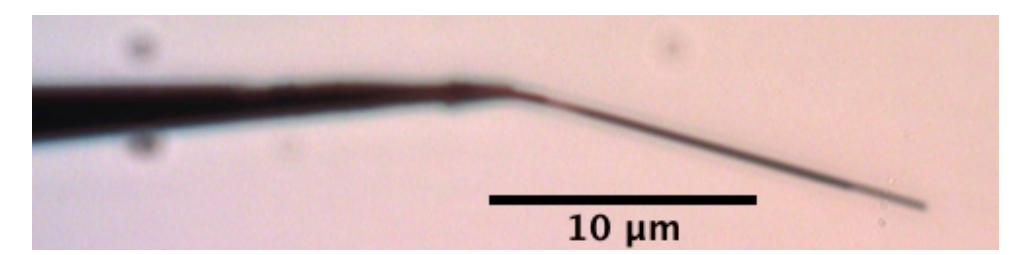

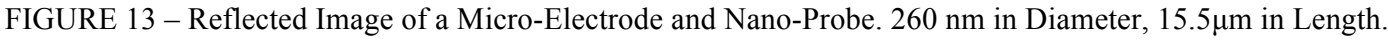


FIGURE 14 - Reflected Image of a Nano-Probe on a Micro-Electrode With a Gallium Droplet. The Nano-Probe is 500 $\mathrm{nm}$ in Diameter, $25 \mu \mathrm{m}$ in Length.

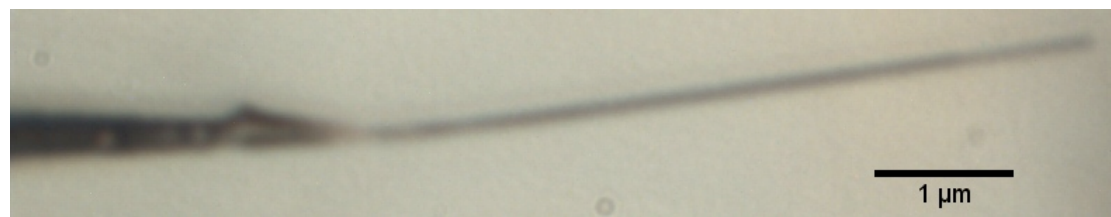

FIGURE 15 - Reflected Image of a Nano-Probe. $150 \mathrm{~nm}$ in diameter, $6.1 \mu \mathrm{m}$ in length.

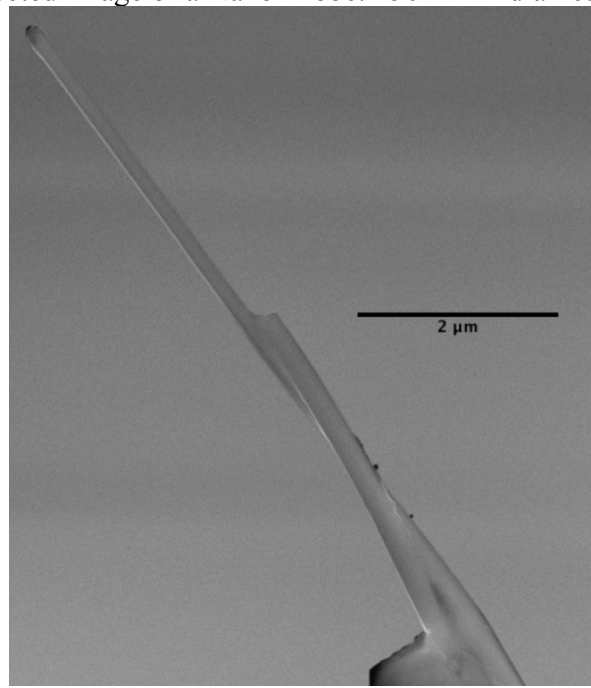

FIGURE 16 - SEM Image of a Gold Coated Nano-Probe. $162 \mathrm{~nm}$ in Diameter, $7.96 \mu \mathrm{m}$ in Length.

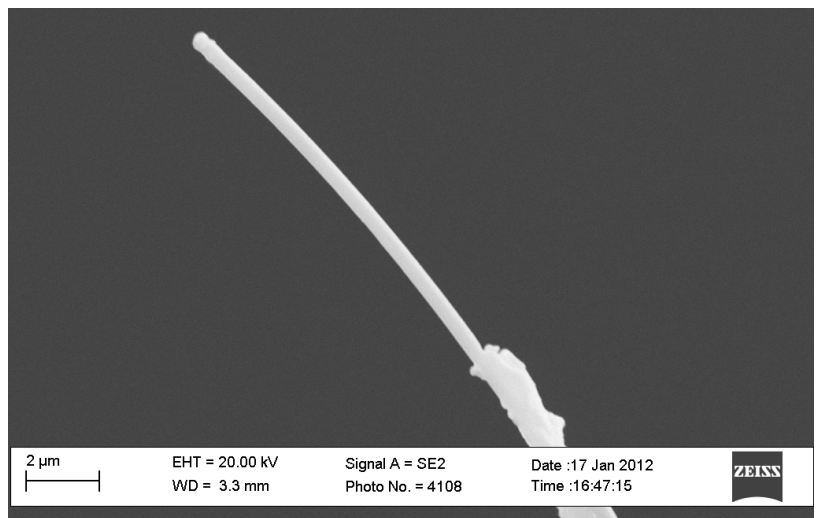

FIGURE 17 - SEM Image of a Gold Coated Nano-Probe. $475 \mathrm{~nm}$ in Diameter, $11.8 \mu \mathrm{m}$ in Length. 


\section{B. Polystyrene Nano-Particles}

Three different sizes of polystyrene particles, with diameters of $200 \mathrm{~nm}, 500 \mathrm{~nm}$, and $1000 \mathrm{~nm}$, were suspended in solutions with various conductivities, $5.5 \mathrm{E}-8 \mathrm{~S} / \mathrm{m}, 2.5 \mathrm{E}-3$ $\mathrm{S} / \mathrm{m}$, and $2.26 \mathrm{E}-1 \mathrm{~S} / \mathrm{m}$. These particles were chosen due to their size, availability, and fluorescent properties. Additionally, polystyrene and latex particles have been widely studied; The parameters controlling the $\mathrm{pDEP}$ and $\mathrm{nDEP}$ forces on polystyrene and latex particles are well defined $[6,14,16,18,19,23,28,33-43,63,85,86]$. The classical form of the DEP equation, given by equation 9 in Section I, shows the relationship between particle volume and the magnitude of the DEP force. In order for the DEP force to dominate the other forces in the system, e.g. ET flow, Brownian motion, etc., a sharp gradient in the electric field must exist. This gradient can be intensified in two ways. One method is to increase the potential across the electrodes, thus increasing the magnitude of the electric field. This is not an ideal solution to create a stronger gradient since Joule heating increases with the square of the potential $\left(\mathrm{V}^{2}\right)$, and the electrothermal flow increases with the potential to the fourth power $\left(\mathrm{V}^{4}\right)$. The other method to increase the gradient in the electric field is to use an electrode configuration which approaches the classic 'point and plane' model by decreasing the size of the electrodes. The nanoprobes, due to their sub-micron features, are an example of the latter method. The small diameter of the nano-probe created a sharp gradient in the electric field capable of easily capturing $200 \mathrm{~nm}$ polystyrene particles with $\mathrm{pDEP}$ at low voltages. Suspending media of various conductivity levels were used to show how the DEP force was affected.

Figure 18 is a time series of photos, taken 0.725 seconds apart, of the capture of 200 $\mathrm{nm}$ fluorescent particles by pDEP forces. Fluorescence increased the visible contrast 
between the particles, the medium, and the nano-probe. The applied AC signal had an amplitude of $0.6 \mathrm{~V}_{\mathrm{rms}}$ and the frequency was $600 \mathrm{kHz}$.

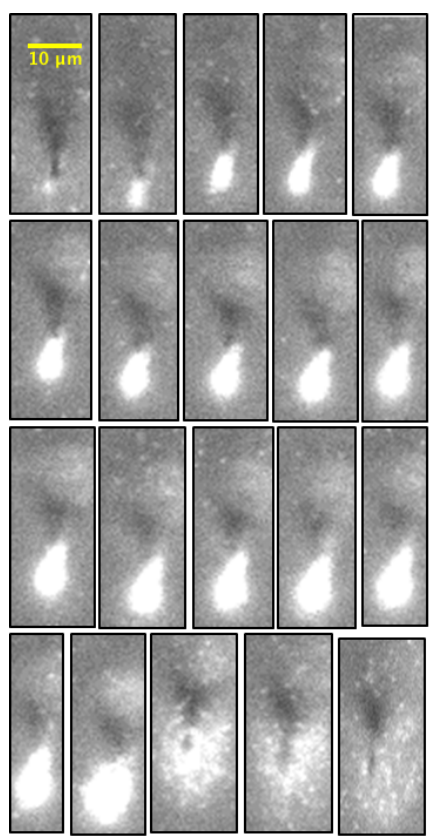

FIGURE 18 - Time Lapse Sequence of the pDEP Capture and Release of $200 \mathrm{~nm}$ Polystyrene Particles Suspended in DI Water. The $\Delta \mathrm{t}$ Between Photos is $0.725 \mathrm{~s}$. The AC Signal had an Amplitude of $0.6 \mathrm{~V}_{\mathrm{rms}}$ and a Frequency of 600 $\mathrm{kHz}$.

Figure 19 shows nDEP particle repulsion as compared to the nano-probe without an applied AC signal. The solution contained $200 \mathrm{~nm}$ polystyrene particles in water with a conductivity of $5.5_{\mathrm{E}-8} \mathrm{~S} / \mathrm{m}$. The applied AC signal had an amplitude of $0.6 \mathrm{~V}_{\mathrm{rms}}$ and a frequency of $6 \mathrm{MHz}$.

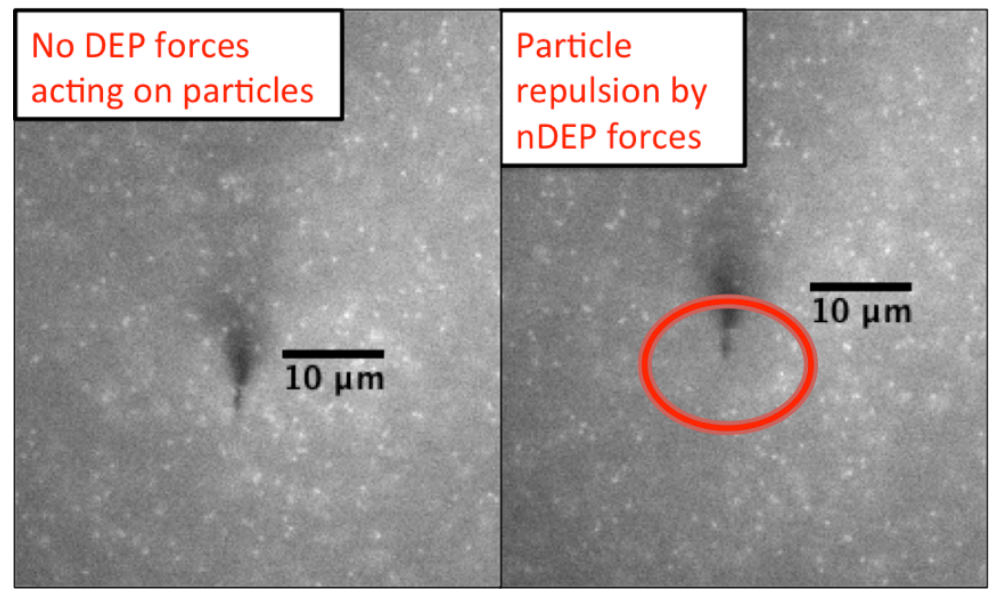

FIGURE 19 - To the Left, No Signal is Applied to the Nano-Probe. To the Right, nDEP Particle Repulsion of $200 \mathrm{~nm}$ Polystyrene Particles Suspended in Water with a Conductivity of $5.5 \mathrm{E}-8 \mathrm{~S} / \mathrm{m}$. The AC Signal had an Amplitude of 6 $\mathrm{V}_{\mathrm{rms}}$ and a frequency of $6 \mathrm{MHz}$. 
Tables II and III contain the applied signal information and whether the particles were attracted or repelled from the nano-probe. This data was taken for a variety of different particle diameters $\left(\mathrm{d}_{\mathrm{p}}\right)$, conductivities $\left(\sigma_{\mathrm{m}}\right)$, potentials $\left(\mathrm{V}_{\mathrm{pp}}\right)$, and frequencies.

TABLE II

EXPERIMENTAL RESULTS

\begin{tabular}{|c|c|c|c|c|}
\hline$d_{p}(n m)$ & $\sigma_{\mathrm{m}}(\mathrm{S} / \mathrm{m})$ & $\begin{array}{l}\text { Amplitude } \\
\left(\mathrm{V}_{\mathrm{pp}}\right)\end{array}$ & $\begin{array}{c}\text { Frequency } \\
(\mathrm{kHz})\end{array}$ & $\begin{array}{l}\text { Dominant Force } \\
\text { on Particles }\end{array}$ \\
\hline \multirow{8}{*}{200} & \multirow{2}{*}{$5.5 \mathrm{E}-8$} & 1 & 600 & pDEP \\
\hline & & 3 & 5000 & nDEP \\
\hline & \multirow{6}{*}{$2.26_{\mathrm{E}-1}$} & 3 & 500 & ET Flow \\
\hline & & \multirow{2}{*}{4} & 500 & ET Flow \\
\hline & & & 1000 & ET Flow \\
\hline & & \multirow{3}{*}{5} & 1000 & ET Flow \\
\hline & & & 3000 & ET Flow \\
\hline & & & 5000 & ET Flow \\
\hline \multirow{2}{*}{500} & \multirow{2}{*}{$5.5 \mathrm{E}-8$} & 1 & 100 & pDEP \\
\hline & & 5 & 100 & pDEP \\
\hline \multirow{4}{*}{1000} & \multirow{3}{*}{$5.5_{\mathrm{E}}-8$} & \multirow{2}{*}{1} & 200 & pDEP \\
\hline & & & 940 & nDEP \\
\hline & & 6 & 10000 & nDEP \\
\hline & $2.2 \mathrm{E}-2$ & 5 & 200 & nDEP \\
\hline
\end{tabular}


TABLE III

DOMINANT FORCES ACTING ON 200 NM POLYSTYRENE PARTICLES AT 0.6 $\mathrm{V}_{\mathrm{RMS}}$

\begin{tabular}{|c|c|c|c|}
\hline \multirow{2}{*}{$\begin{array}{c}\text { Frequency } \\
(\mathrm{kHz})\end{array}$} & \multicolumn{3}{|c|}{$\sigma_{\mathrm{m}}(\mathrm{S} / \mathrm{m})$} \\
\cline { 2 - 4 } & $5.5_{\mathrm{E}-}-8$ & $2.5_{\mathrm{E}-3}$ & $2.26_{\mathrm{E}-}-1$ \\
\hline 1 & - & ET Flow & ET Flow \\
\hline 5 & ET Flow & ET Flow & ET Flow \\
\hline 10 & ET Flow & ET Flow & ET Flow \\
\hline 50 & ET Flow & - & - \\
\hline 100 & ET Flow & ET Flow & ET Flow \\
\hline 400 & pDEP & - & - \\
\hline 500 & pDEP & pDEP & ET Flow \\
\hline 1000 & pDEP & pDEP & ET Flow \\
\hline 5000 & pDEP & none & ET Flow \\
\hline 10000 & - & nDEP & - \\
\hline 15000 & nDEP & - & - \\
\hline 50000 & - & - & nDEP \\
\hline
\end{tabular}

In general, and independent of solution conductivity, the collected data shows that pDEP forces were exerted on polystyrene particles when a signal with a low frequency (less than $1 \mathrm{MHz}$ ) was applied. nDEP forces were exerted on the same particles when a signal with a higher frequency was applied. DEP forces must dominate the other forces in the system in order to completely dictate particle movement. The DEP experiments initially used particles with diameters of $1000 \mathrm{~nm}$ diameter particles, then $500 \mathrm{~nm}$ particles in the next set of experiments, and finally $200 \mathrm{~nm}$ particles. By decreasing the diameter of the particles, the nano-probe proved that it was capable of creating an electric field gradient strong enough to capture or repel very small polystyrene particles with DEP forces.

Recall that the DEP force is proportional to the volume (radius cubed) of the particle (equation 9). Therefore, the DEP force acting on the particles decreases by a factor of 8 
between the $1000 \mathrm{~nm}$ particles and the $500 \mathrm{~nm}$ particles, a factor of 15.625 between the $500 \mathrm{~nm}$ particles and the $200 \mathrm{~nm}$ particles, and the DEP force acting on the $200 \mathrm{~nm}$ particles is 125 times less than the DEP force acting on the $1000 \mathrm{~nm}$ particles. The decreasing volume of the polystyrene particles requires the electric field strength to triple between 1000 and 500, increase by a factor of 4 for the particles between $500 \mathrm{~nm}$ particles and the $200 \mathrm{~nm}$ particles, while the electric field must be 11 times stronger to create the same DEP magnitude on the $200 \mathrm{~nm}$ particles as the $1000 \mathrm{~nm}$ particles. This data is summarized in Table IV.

TABLE IV

REQUIRED ELECTRIC FIELD INCREASE FOR DECREASING PARTICLE DIAMETER

\begin{tabular}{|c|c|c|}
\hline $\begin{array}{c}\text { Diameter Ratio of Large } \\
\text { to Small Particles }\end{array}$ & DEP force decrease & Required |E $\mid$ increase \\
\hline 2 & 8.0 & 2.83 \\
\hline 2.5 & 15.6 & 3.95 \\
\hline 5 & 125.0 & 11.2 \\
\hline
\end{tabular}

Equations 14-17 show that the strong dependence on DEP force due to the conductivity of the solution as well as the applied frequency of the signal. These relationships are expressed through the $\mathrm{CM}$ factor. When the polystyrene particles were suspended in low conductivity solutions and an AC signal with a low frequency is applied, the pDEP force is the most dominant force in the system. An AC signal with a high frequency applied to the same system will produce nDEP forces. Experimental results in Tables II and III confirmed these trends.

As the conductivity of the suspending medium changed, so did the dominant forces in the system. The Joule heating at the tip of the nano-probe is linearly proportional with 
the conductivity of the solution. The change in temperature, $\Delta \mathrm{T}$, may cause convection currents and the resulting fluid motion could dislodge particles attracted by pDEP forces. This localized temperature increase was not an issue since the increase was numerically simulated to be less than $1{ }^{\circ} \mathrm{C}$ (see Section III D). However, the temperature was responsible for other effects in the system. The $\Delta \mathrm{T}$ was localized at the end of the nanoprobe, which created a sharp temperature gradient. The resulting electrothermal flow was often detrimental to the system. The flow induced by the temperature gradient was in some cases strong enough to completely destroy the nano-probe. An example of this fluid flow can be seen in Figure 20. Electrothermal flow velocity is proportional to $\sigma_{\mathrm{m}}$ and $\mathrm{V}^{4}$ [51]. This relationship stresses the importance of applying a signal with a small potential. Table V shows the distance of the particles from the nano-probe, as well as the velocity and acceleration of the particles in proximity to the nano-probe. 

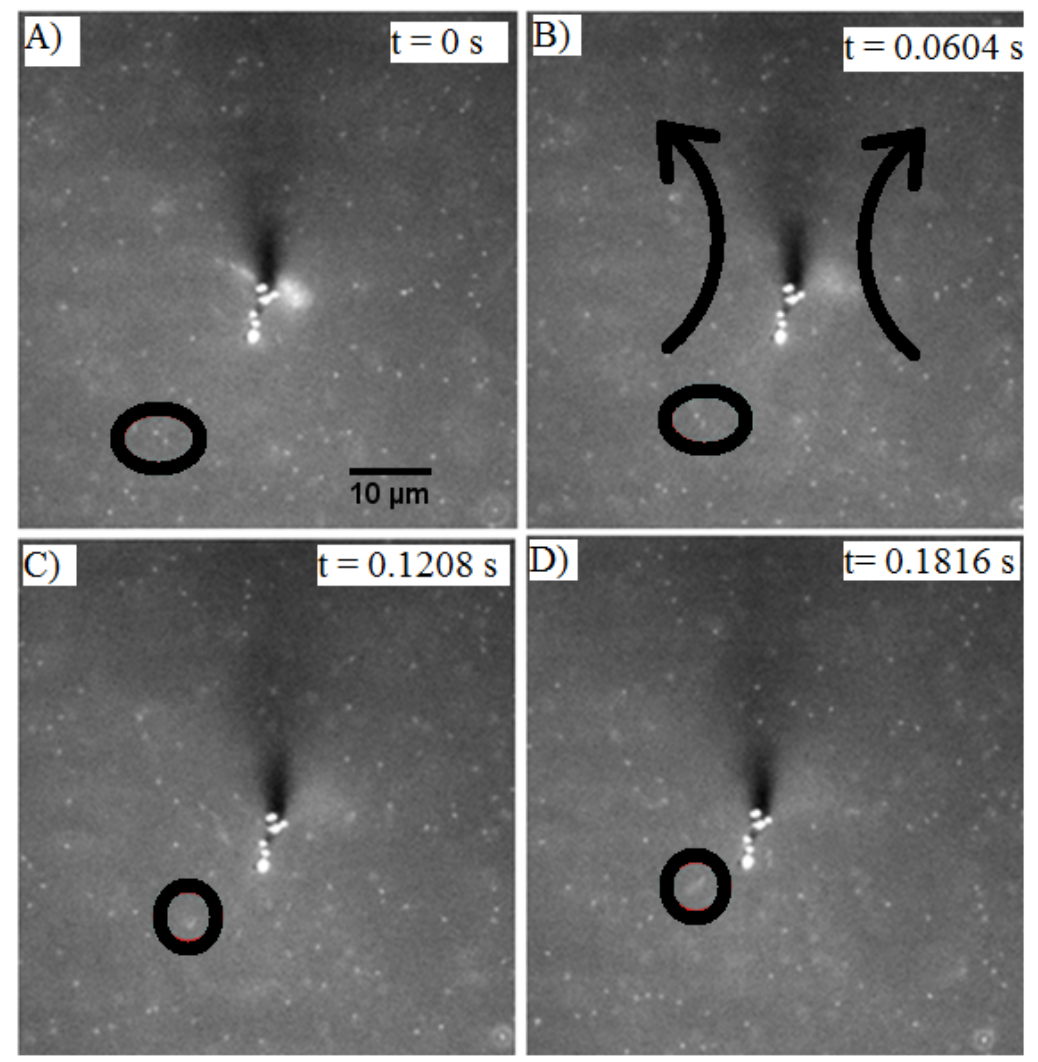

FIGURE 20 - Electrothermal Flow

TABLE V

POLYSTYRENE NANO-PARTICLE MOTION DUE TO ET FLOW

\begin{tabular}{|c|c|c|}
\hline Figure & $\begin{array}{c}\text { Distance from } \\
\text { Nano-Probe }(\mu \mathrm{m})\end{array}$ & $\mathrm{v}(\mu \mathrm{m} / \mathrm{s})$ \\
\hline A & 16.1 & \\
\hline B & 13.6 & 41.1 \\
\hline C & 10.9 & 45.3 \\
\hline D & 5.02 & 97.2 \\
\hline
\end{tabular}

Equations 9-17 show the relationship between particle conductivity, medium conductivity, the frequency of the applied $\mathrm{AC}$ signal and the $\mathrm{CM}$ factor. This relationship implies that there exists some cross-over frequency, $f_{c o}$, where the $\mathrm{CM}$ factor is 0 . The crossover frequency for the polystyrene particles was determined by experimentation to be $3 \mathrm{MHz}$ and $6 \mathrm{MHz}$ for a media conductivity of $5.5 \mathrm{E}-8$ and $2.5 \mathrm{E}-3$ $\mathrm{S} / \mathrm{m}$, respectively. The surface charge of the polystyrene particles changes with the 
conductivity of the medium $[26,85]$, and greatly affected the overall conductivity of the particle. Based on the experimentally determined cross-over frequencies, the conductivity of the polystyrene particles was $2.98_{\mathrm{E}-3}$ and $5.79_{\mathrm{E}-3}$ for the DI water, $\sigma_{\mathrm{m}}=5.5_{\mathrm{E}-} 8 \mathrm{~S} / \mathrm{m}$,

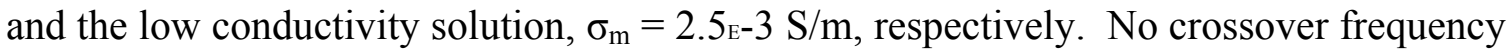
was determined for the high conductivity solution, $\sigma_{\mathrm{m}}=2.26_{\mathrm{E}-1} \mathrm{~S} / \mathrm{m}$.

Figures 21-23 for show plots of the theoretical $\operatorname{Re}[\mathrm{CM}]$ for various conductivities and particle properties. The solid line represents the theoretically calculated $\operatorname{Re}[\mathrm{CM}]$ value (equation 14). The experimentally observed ET flow region, pDEP region, and nDEP regions have been superimposed over the theoretical $\mathrm{Re}[\mathrm{CM}]$ value. The experimental results are consistent with the theory supplied by Pohl [1] and Jones [2].

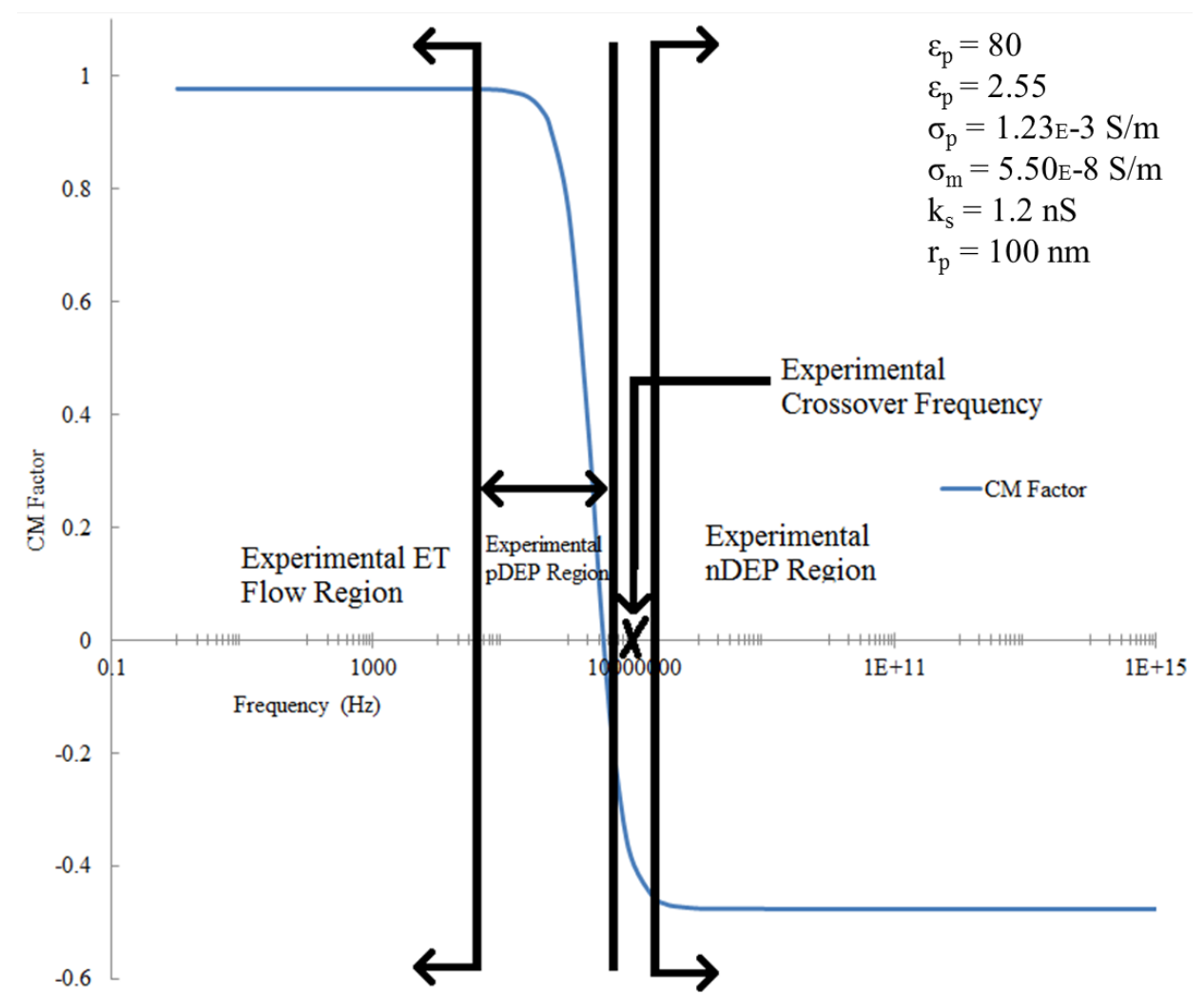

FIGURE 21 - CM Factor as a Function of Frequency for Polystyrene Particles Suspended in DI Water. 


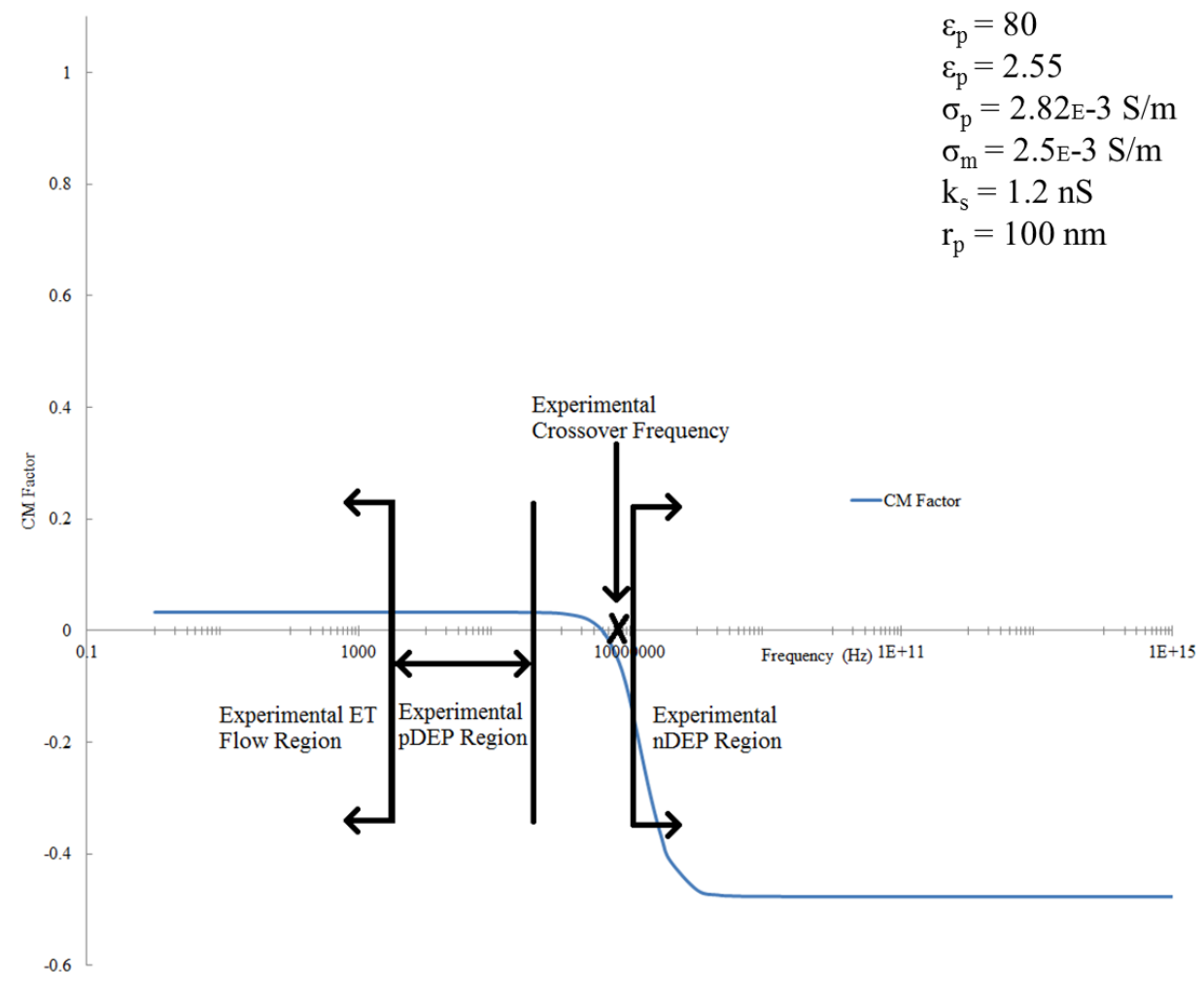

FIGURE 22 - CM Factor as a Function of Frequency for Polystyrene Particles Suspended in a Low Conductivity Solution

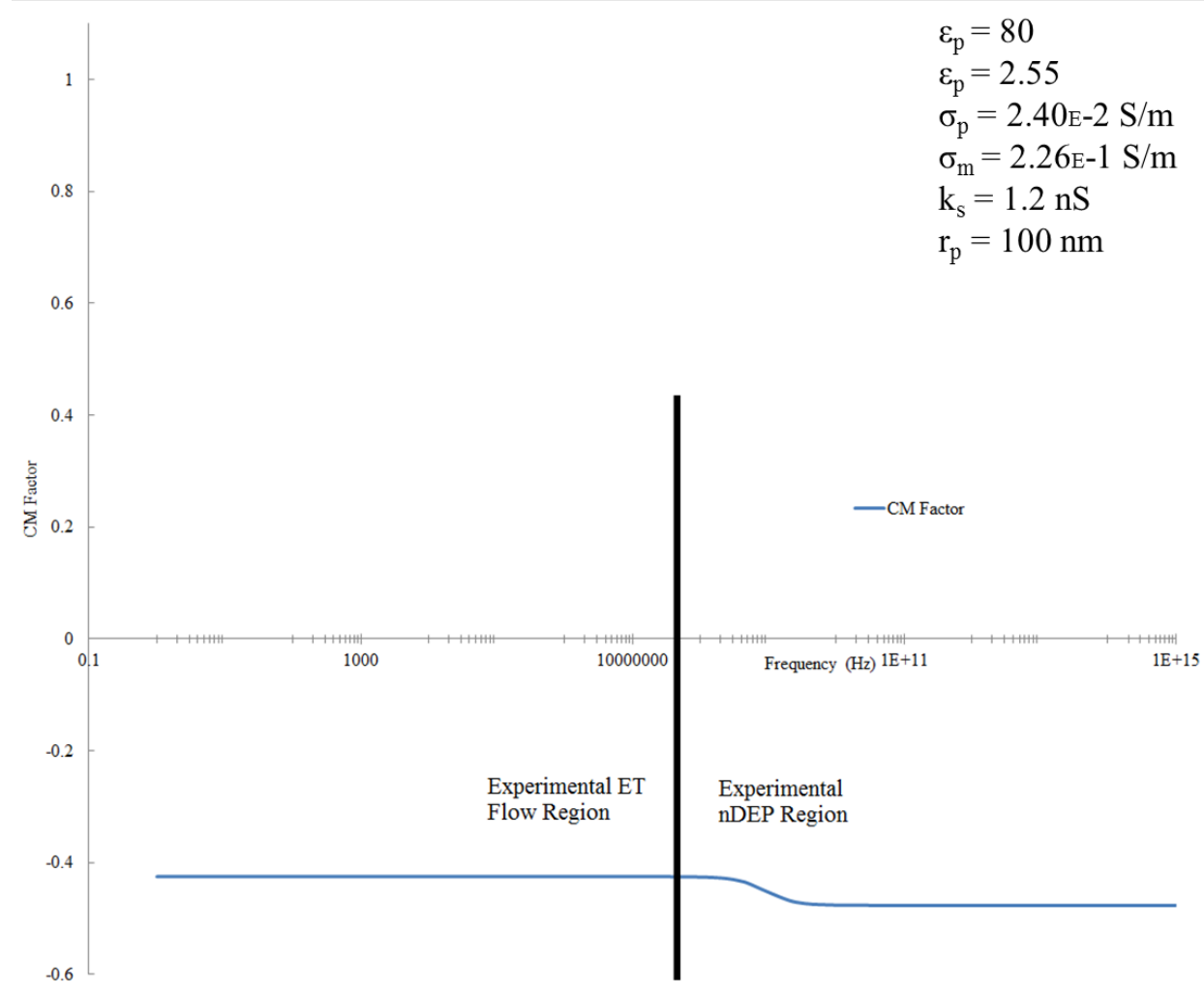

FIGURE 23 - CM Factor as a Function of Frequency for Polystyrene Particles Suspended in a High Conductivity Solution 


\section{Gold Nano-Particles}

The nano-probe and ITO coverslip electrodes proved to be capable of capturing polystyrene particles as small as $200 \mathrm{~nm}$. Smaller gold nano-particles were tested to determine the lower size limit capable of being captured by the nano-probe electrode. Two different sets of gold nano-particles were suspended in DI water. The larger gold nano-particles were spherical with diameters of approximately $120 \mathrm{~nm}$. The smaller set of gold nano-particles consisted of a variety of crystalline structures whose characteristic length ranged from 5-50 nm. The latter group of gold nano-particles had hexagonal crystals, cylindrical rods, as well as various tetrahedron crystals.

The gold nano-particles were estimated to have a conductivity of approximately 4.10E7 $\mathrm{S} / \mathrm{m}$. The high conductivity of the gold resulted in only pDEP forces being present in the system because the gold nano-particles were always more conductive than the suspending medium. Studies by Gierhart, et al. [28], showed that the cross-over frequency for gold in deionized water was near $10^{18} \mathrm{~Hz}$, which far exceeded the limitations of the experimental setup in these studies. The CM factor was calculated to be approximately 0.98 for all frequencies from $1 \mathrm{~Hz}$ to $100 \mathrm{MHz}$.

Experimental results with the large spherical gold shells were similar to the results of the experimental results with the polystyrene nano-particles. The gold shells were drawn to the end of the nano-probe, thus demonstrating that pDEP forces were strong enough to overcome all other forces, e.g. Brownian motion, etc., in the system. These particles agglomerated in several locations near the end of the probe as shown in Figure 24. However, unlike the polystyrene nano-particles, some of the gold shells were rigidly 
attached to the end of the nano-probe. This effect is known as pearl chaining, which has been studied in-depth [87].

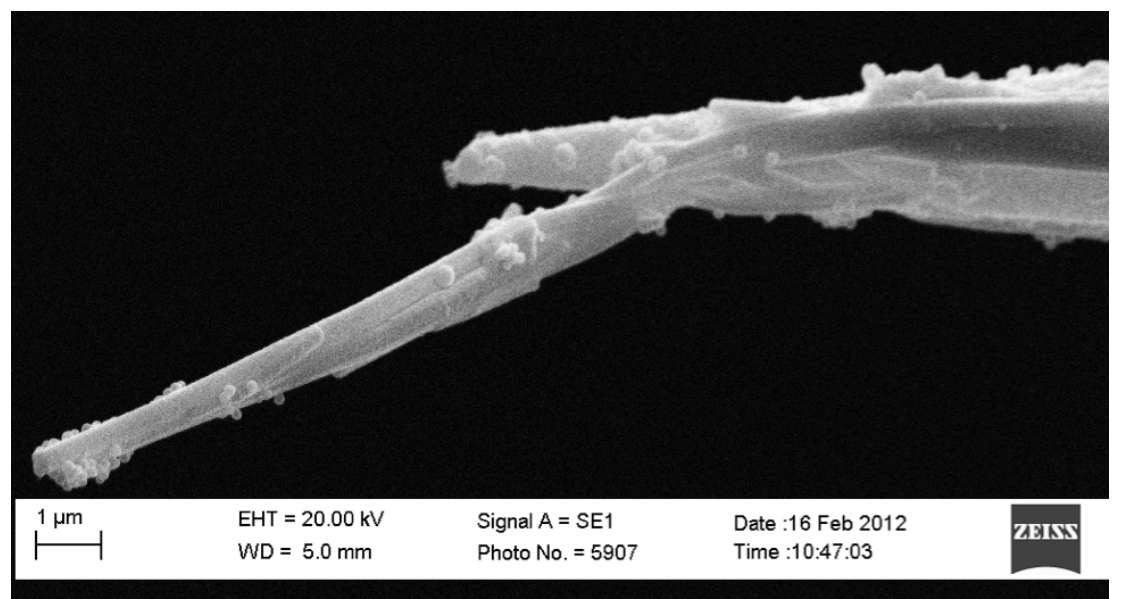

FIGURE 24 - Nano-Probe with Gold Nano-Shells Adhered by pDEP

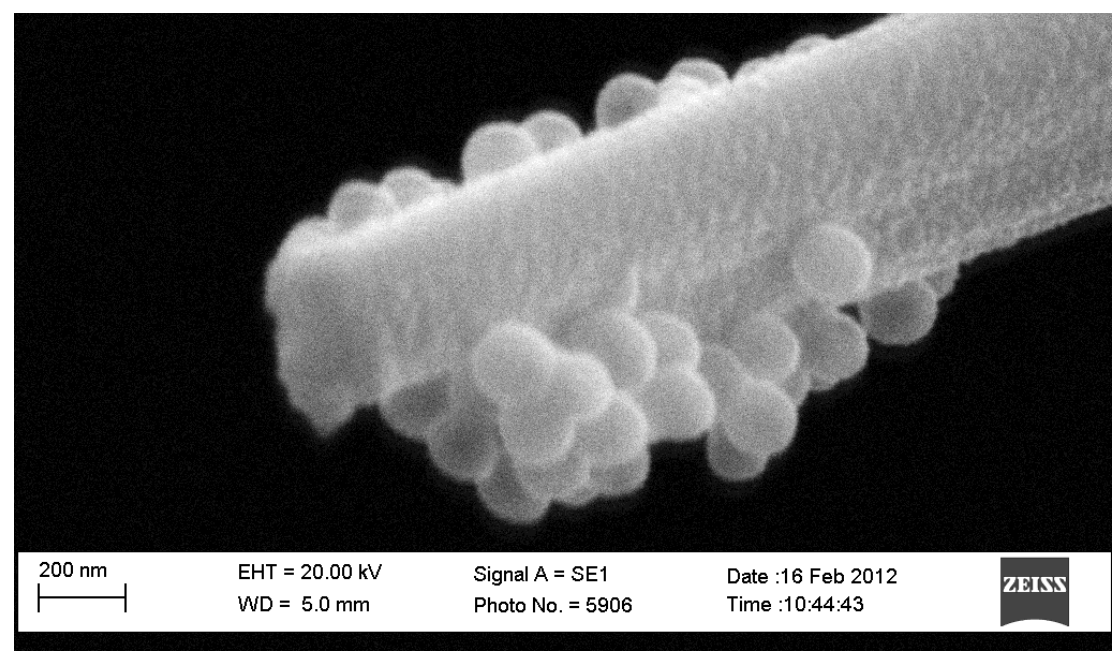

FIGURE 25 - Enlarged View of Nano-Probe and Attached Gold Nano-Shells

The smaller, assorted gold nano-particles were interesting to study because of their size. Previous DEP force studies have been done with electrodes manufactured using standard lithography techniques $[5,6,14-16,18,19,21,25,28,30,33,37,40,42,43,50$, $51,59,63,67,86-93$ ] were capable of creating strong DEP forces. Results by Li, et al. demonstrated the capture of $2 \mathrm{~nm}$ gold particles [31] on five micron wide planar electrodes with rounded ends. The nano-probes used in this work captured particles as 
small as $5 \mathrm{~nm}$ with a much lower applied voltage. Li, et al. used an AC signal with an amplitude of $16 \mathrm{~V}_{\mathrm{pp}}$, whereas the experiments herein used an $\mathrm{AC}$ signal with a maximum amplitude of approximately $1 \mathrm{~V}_{\text {rms. }}$.

Similar to the larger gold nano-particles, the smaller gold particles were attached to the nano-probe, except the agglomeration of the particles was highly anisotropic. The larger gold shells accumulated uniformly around the end of the nano-probe, while the smaller particles appeared to assemble in fractal patterns that extended radially around the nano-probe. The larger gold shells were primarily captured near the end of the nanoprobe, but the smaller gold particles often assembled on the nano-probe, as well as higher up the micro-electrode. This particle assembly can be seen in Figures 26-27.

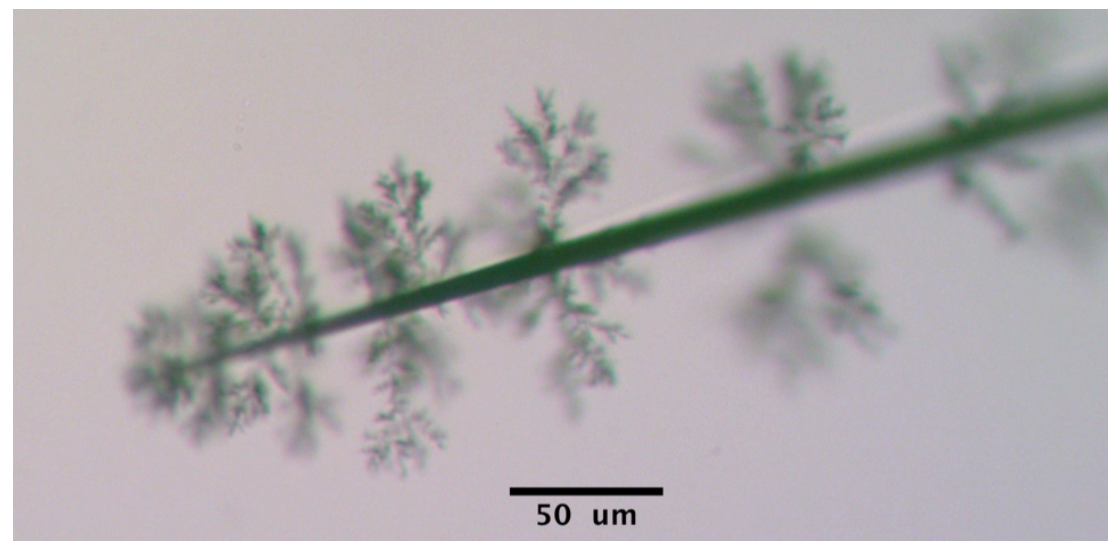

FIGURE 26 - Gold Nano-Particles Grown on Capillary Tube and Nano-Probe

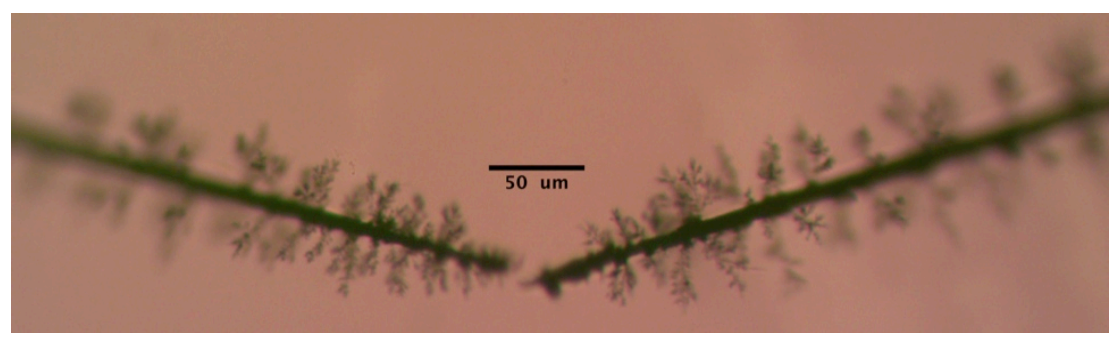

FIGURE 27 - Dual Nano-Probes with Gold Nano-Particles Grown

The assembly of the smaller set of gold particles, shown in Figures 26 and 27, was most likely due to pearl chaining [87]. In most experiments, the two electrodes were the 
nano-probe and the ITO coated cover slip. In a few other experiments, two nano-probes were used as electrodes. If the electrodes were a relatively large distance apart when the AC signal was applied, the nano-particles created a bridge with a large diameter between the electrodes. If the electrodes were relatively close together when the AC signal was applied, the nano-particles assembled into a bridge with a much thinner bridge. For example, when the distance between electrodes was approximately $5 \mu \mathrm{m}$, the bridge diameters were measured to be approximately $700 \mathrm{~nm}$, shown in Figure 28 . When the distance between the electrodes was much larger, the diameter of the bridge was an nearly an order of magnitude higher. The pearl chaining between the two nano-probes primarily followed the electric field lines. Once the gold bridge had connected the two electrodes, the applied signal was shorted and no further pDEP effects were observed. This bridging effect is shown in Figures 28 and 29.

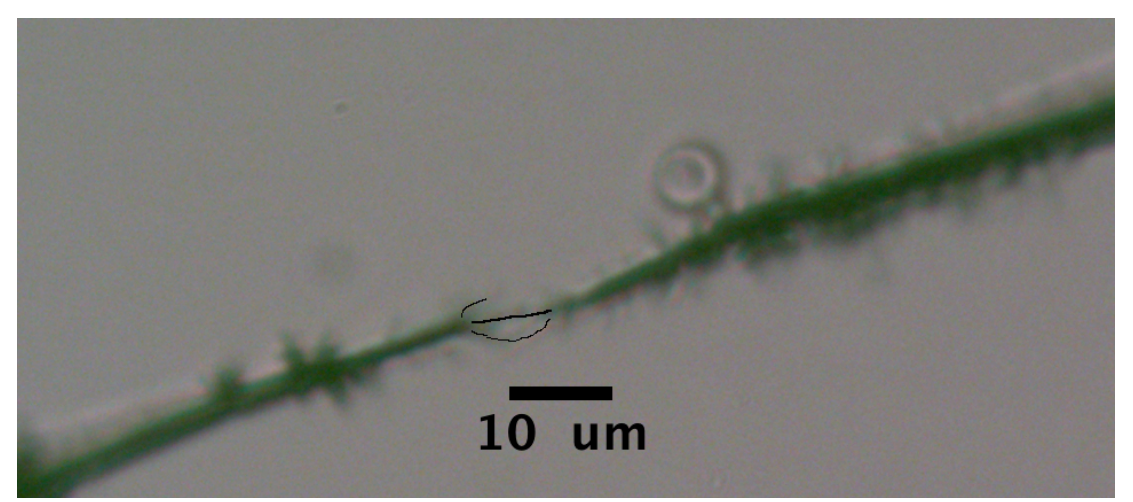

FIGURE 28 - Gold Nano-Particle Bridge Between 2 Nano-Probes. The Bridge is Outlined in Black to Enhance Contrast. 


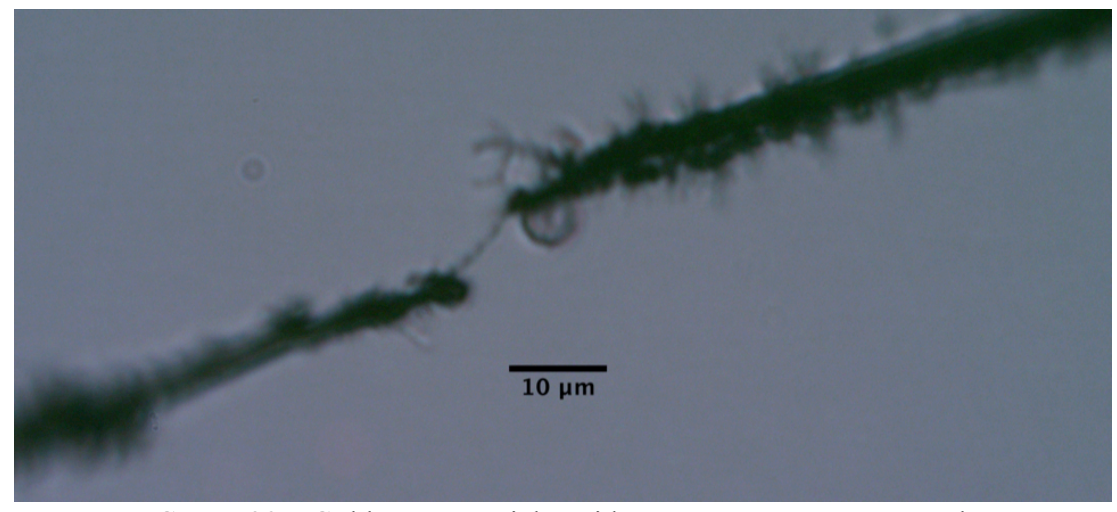

FIGURE 29 - Gold Nano-Particle Bridge Between Two Nano-Probes

As previously discussed, pDEP polystyrene particles were drawn to the end of the nano-probe. When the AC signal was shut off, the polystyrene particles were no longer held in place by pDEP forces and disseminated accordingly. This was not the case with the gold nano-particles. The smaller gold particles fused together to create long chains, while the larger gold shells fused together near the end of the nano-probe. The collection of particles via pDEP forces was visible under an optical microscope although neither the individual gold shells nor the smaller gold crystals were visible in solution. These gold nano-particle assemblies were rigidly attached to both electrodes. Kretschmer et al. [87] determined that currents above $1 \mathrm{~mA}$ fused gold nano-particles. Their studies noted that the individual particles fused together in areas where many particles had been collected, and that the individual particles were resolvable in the regions with few particles. Kretschmer et al. [87] described this as "local melting under preservation of the overall shape."

These unique formations stayed rigidly attached to the nano-probe and microelectrode for the duration of the experiments. However, the formations were broken off of the nano-probe when it was retracted from the droplet of water due to the surface tension of the water. Several solutions to this problem were tested, and the best results were obtained when a small amount of isopropyl alcohol was added to the water drop to 
act as a surfactant. The surviving nano-probes were placed in the SEM for further observation. These images can be seen in Figures 30-34.

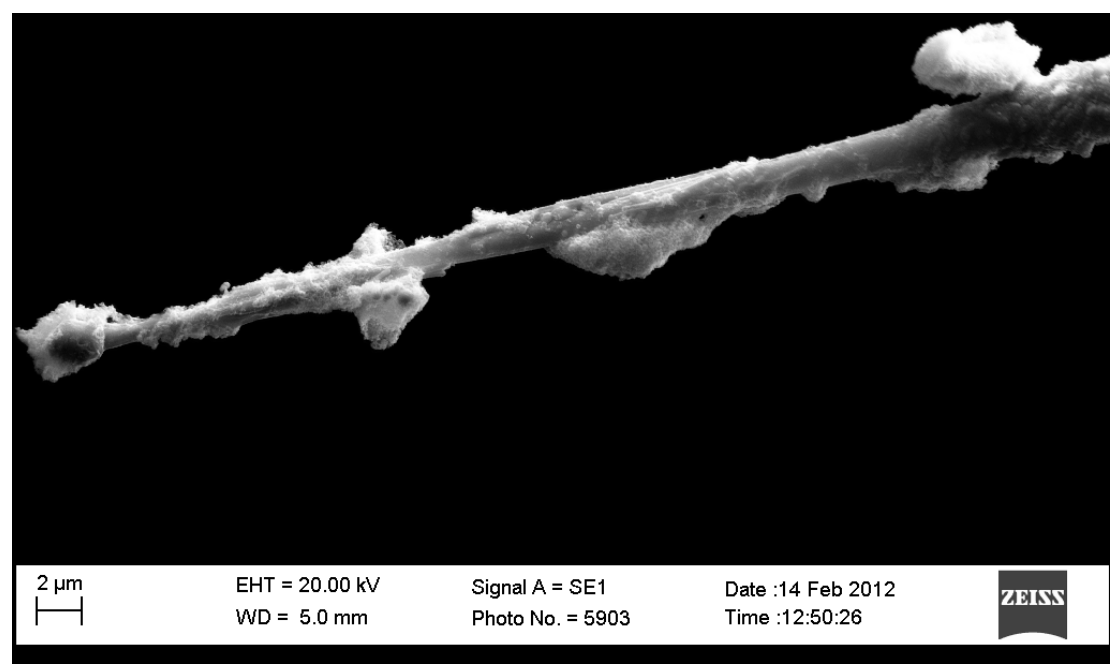

Figure 30 - SEM Image of Gold Nano-Particles Captured by DEP

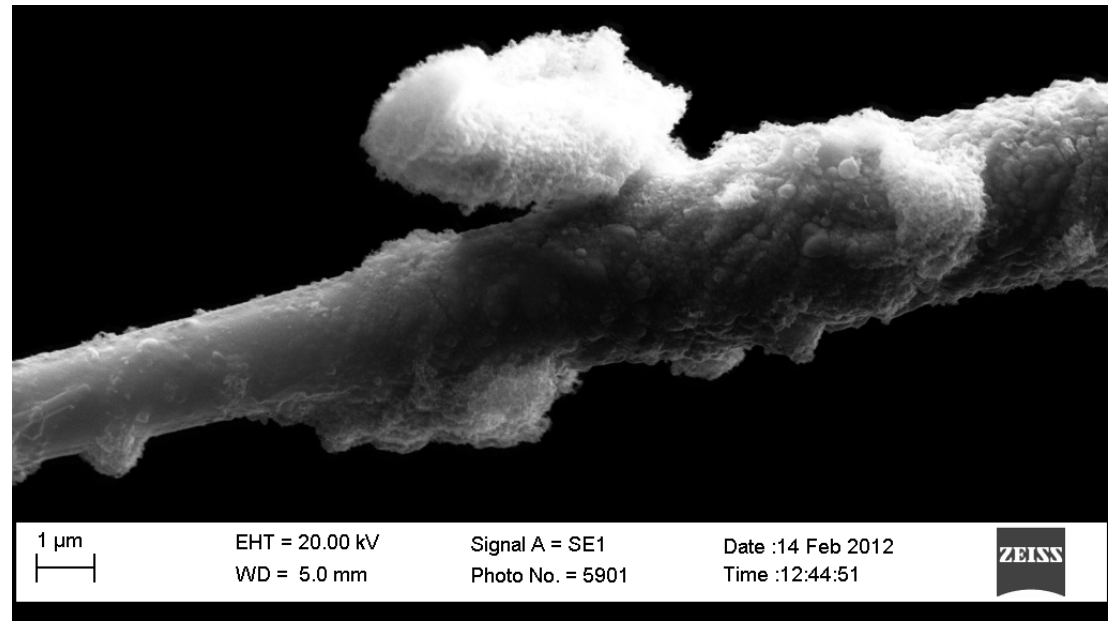

Figure 31 - SEM Image of a Gold Nano-Particle Cluster 


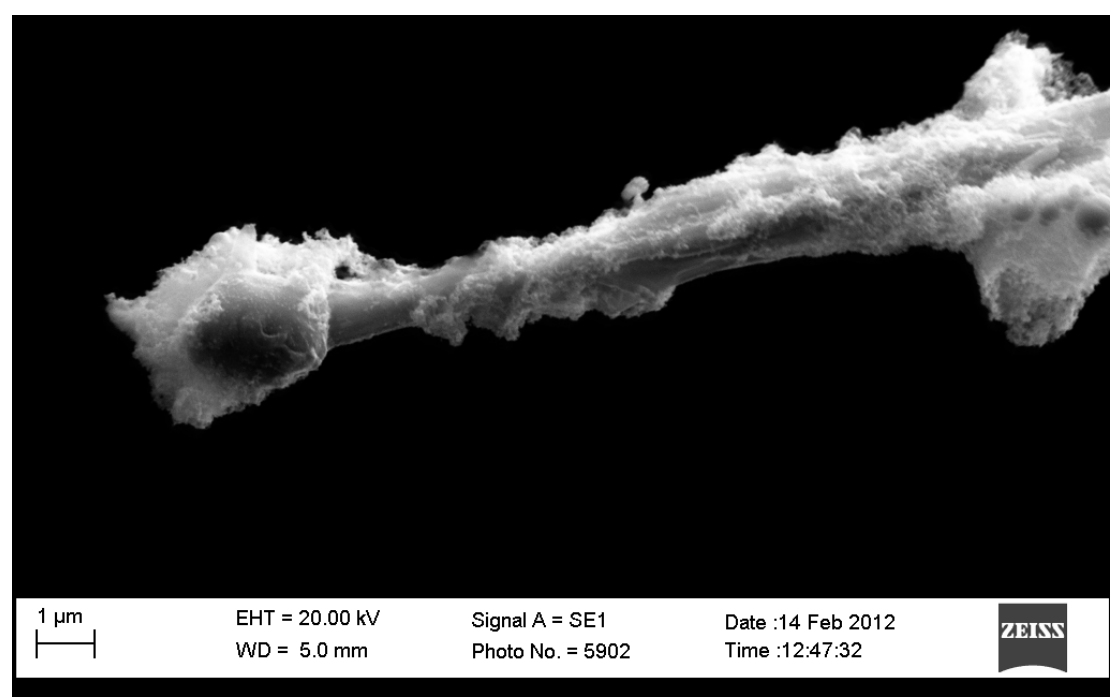

Figure 32 - SEM Image of Gold Nano-Particles Fused to the Nano-Probe by pDEP

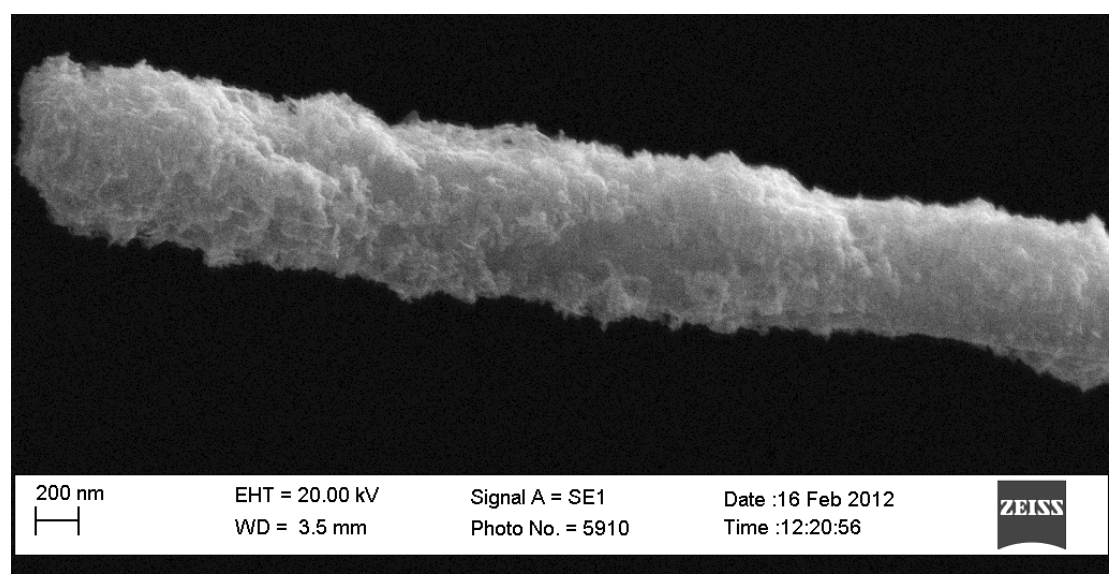

FIGURE 33 - Gold Nano-Particles Adhered to Nano-Probe by pDEP

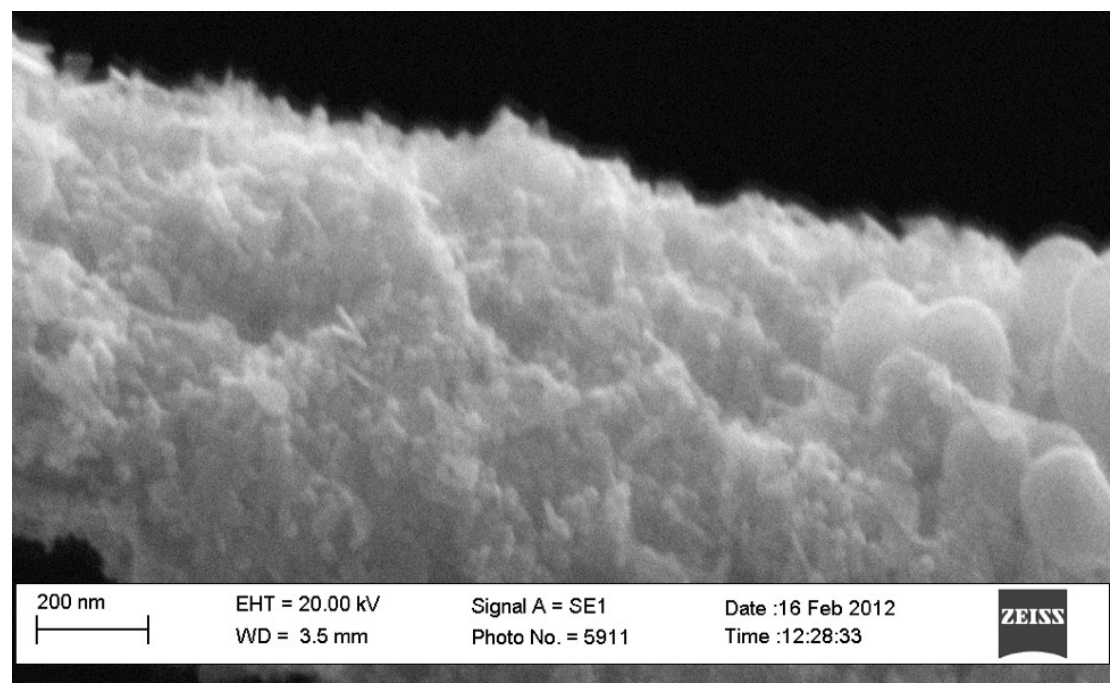

FIGURE 34 - Enlarged View of Gold Nano-Particles Adhered to Nano-Probe by pDEP 


\section{Simulations}

\section{Current Nano-Probe Design}

COMSOL MULTIPHYSICS V3.5 was used to model the nano-probe and ITO coverslip. The numerical models were solved with an axi-symmetric model in two dimensions. Figure 35 shows a schematic of the nano-probe and ITO coverslip as modeled in COMSOL. The distance between the end of the nano-probe and the ITO coverslip was modeled to be $40 \mu \mathrm{m}$, and the nano-probe diameter was modeled to be $250 \mathrm{~nm}$. The horizontal solution space in each model extended $100 \mu \mathrm{m}$ from the origin. The particle diameter was held constant at $200 \mathrm{~nm}$ for all simulations.

An electrical model and a thermal model were numerically solved in the simulations. These models were decoupled, and the electrical model was solved prior to the thermal model. The numerical model was solved sequentially because the system was heated due to the presence of an electric field. Laplace's Equation gives the governing equation for the electrical model

$$
\nabla^{2} \phi=0, E=-\nabla \phi
$$

where $\phi$ is the electric potential. The governing equation for the thermal model can be expressed as a form of Poisson's Equation,

$$
k \nabla^{2} T=-\sigma_{m}\left\langle\boldsymbol{E}^{2}\right\rangle
$$

where $k$ is the thermal conductivity of the medium. The boundary conditions denoted by

$\frac{\delta \phi}{\delta \mathrm{n}}=0$ and $\frac{\delta \mathrm{T}}{\delta \mathrm{n}}=0$ in Figure 35 indicate the presence of an electrical and thermal insulating boundary, respectively. 


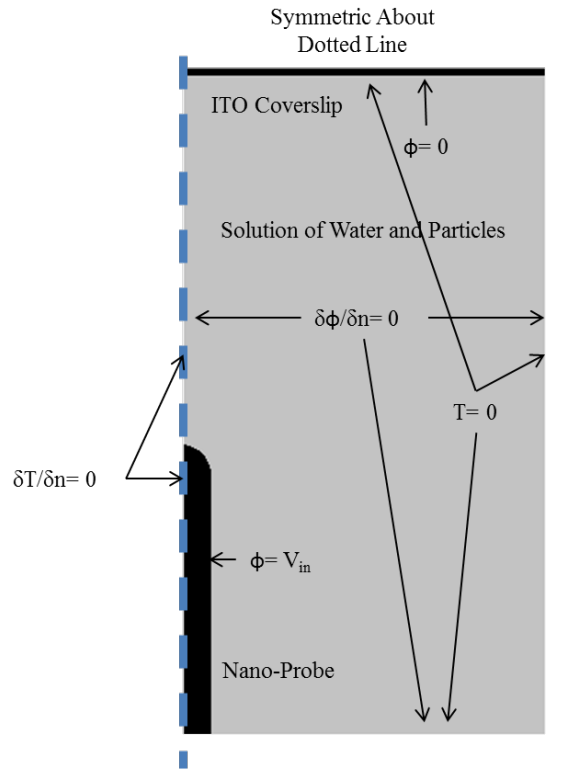

FIGURE 35 - Schematic of Nano-Probe and ITO Coverslip with Boundary Conditions

Figure 36 shows the maximum temperature as a function of the RMS voltage and the conductivity of the medium. Figure 37 shows the maximum temperature gradient as a function of RMS voltage and the conductivity of the medium. The temperature rise and temperature gradient increase with both conductivity and RMS voltage.

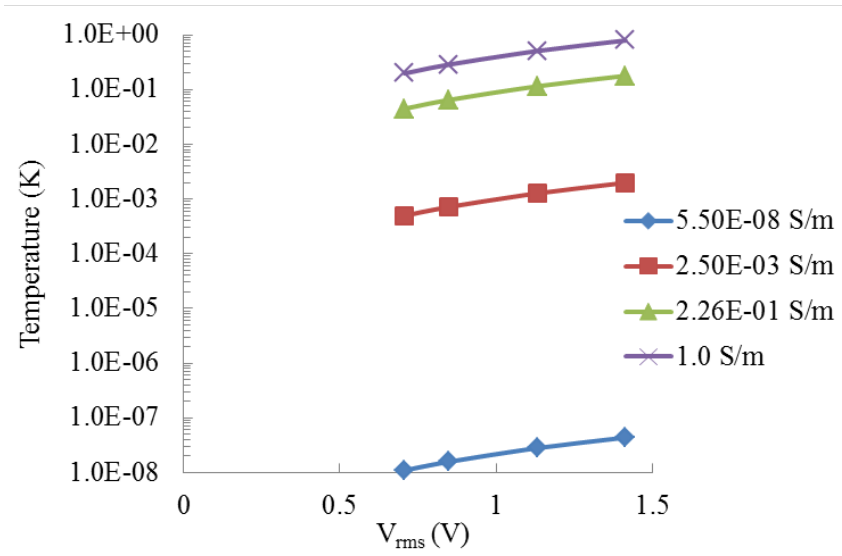

FIGURE 36 - Maximum Temperature Increase as a Function of Voltage and the Conductivity of the Medium 


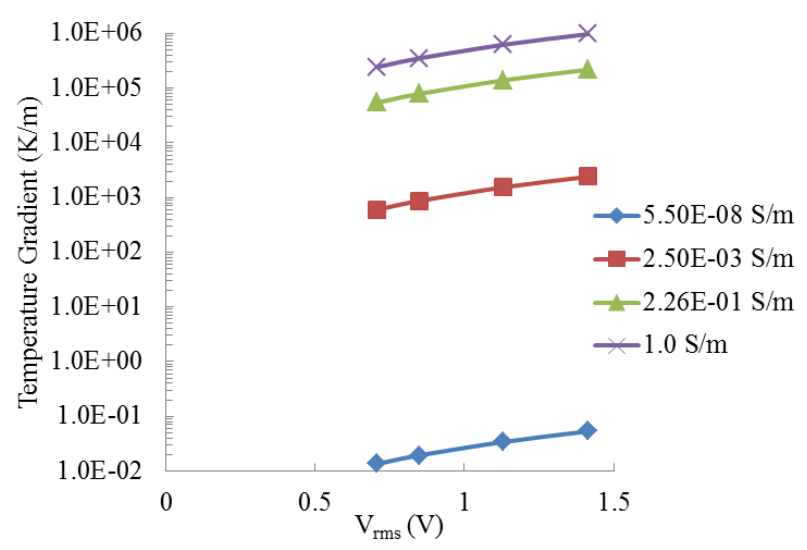

FIGURE 37 - Maximum Temperature Gradient as a Function of Voltage and the Conductivity of the Medium

The trapping radius of the nano-probe was simulated as a function of the applied voltage (Figure 38). The trapping radius was calculated as the difference between the velocity due to the DEP force and the velocity due to the Brownian motion.

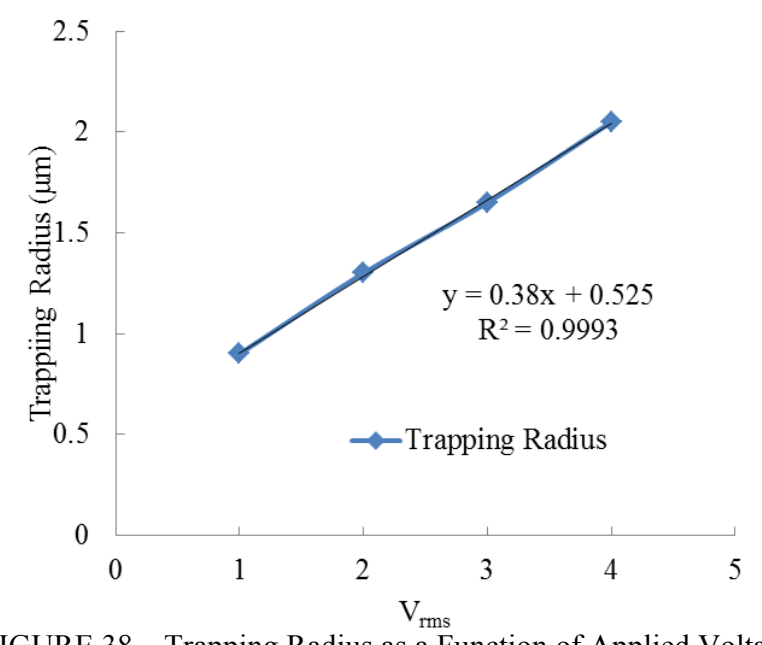

FIGURE 38 - Trapping Radius as a Function of Applied Voltage

The simulations strongly supported the results of the experiments. When the polystyrene nano-particles were suspended in a low conductivity solution and a frequency of less than $6 \mathrm{MHz}$ was applied, a pDEP force was shown. Figure 39 shows an example of the pDEP forces acting on particles with an AC signal of $0.707 \mathrm{~V}_{\mathrm{rms}}$ and a frequency of $1 \mathrm{kHz}$. Figure 40 shows an example of nDEP forces acting on particles with and $\mathrm{AC}$ signal of $0.707 \mathrm{~V}_{\mathrm{rms}}$ and a frequency of $10 \mathrm{MHz}$. Figures 39 and 40 both use particles suspended in DI water with a conductivity of $5.5 \mathrm{E}-8$. The surface plot is 
displayed as the $\log _{10}$ of the magnitude of the DEP force, which was given in Newtons. The arrow plots are normalized and show direction only.

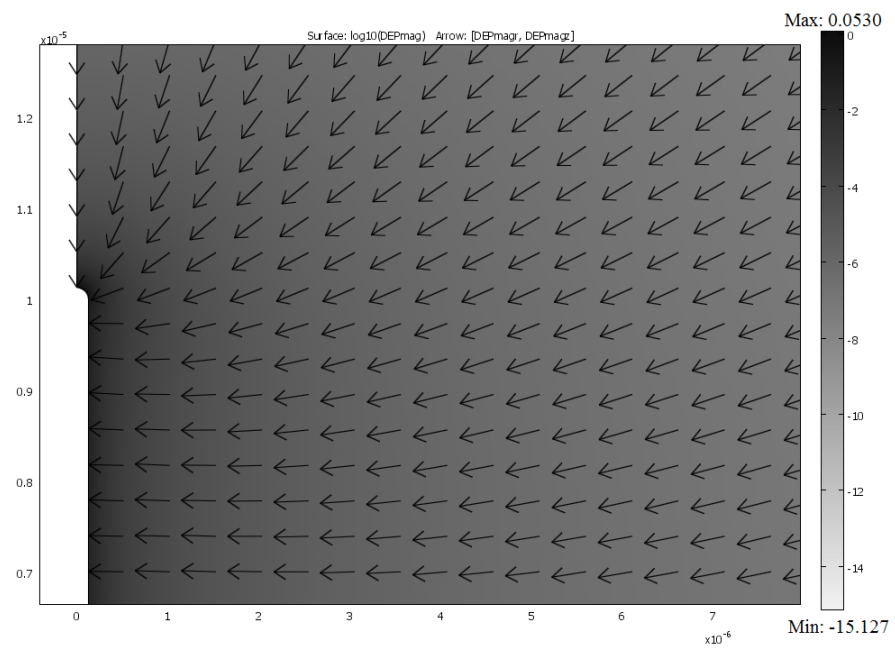

FIGURE 39 - pDEP Near the Tip of the Nano-Probe. $0.707 \mathrm{~V}_{\mathrm{rms}}, 1 \mathrm{kHz}$

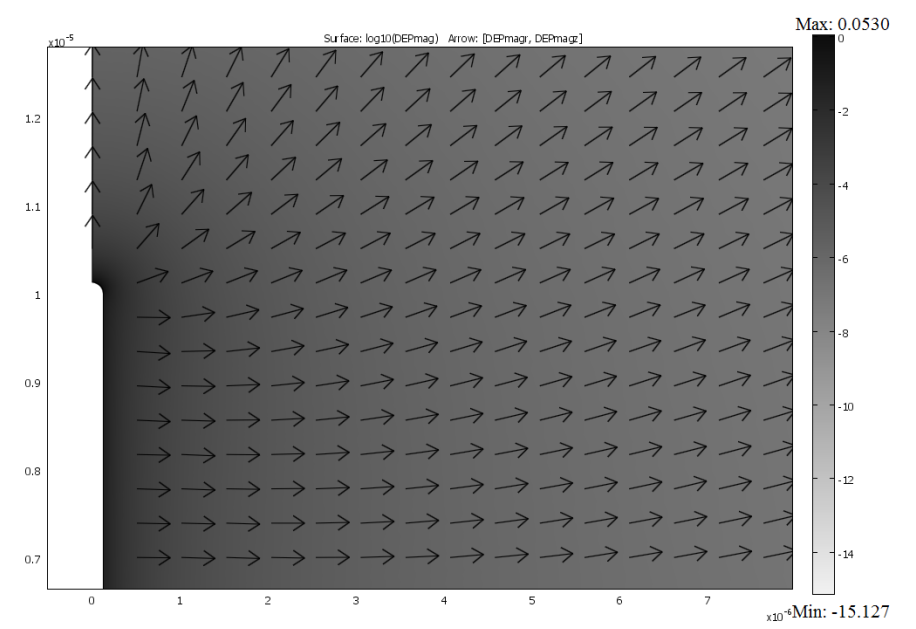

FIGURE 40 - nDEP Near the Tip of the Nano-Probe. $0.707 \mathrm{~V}_{\mathrm{rms}}, 10 \mathrm{MHz}$

\section{2. $\quad$ Revised Nano-Probe Designs}

Alternate nano-probe designs and configurations were sought based off of the results of the nano-probe and ITO cover slip in the high conductivity solution. In the highest conductivity solution, $2.26 \mathrm{E}-1 \mathrm{~S} / \mathrm{m}$, only nDEP was capable of being observed since the conductivity of the medium was greater than the conductivity of the particles. The conductivity of cytoplasm of mammalian cells has been estimated as $1 \mathrm{~S} / \mathrm{m}$ [44], so a 
nDEP trap, similar to the studies by Brown, et al. [63] might be utilized to capture subcellular entities, which are likely to be less conductive than the cytoplasm.

Four different geometries were simulated and their results are shown in Figures 4144. An illustration of each of the four nano-probe geometries is shown in the top-left corner of each page (A). Simulated electric fields are shown in B and C. The simulated trapping region for a $200 \mathrm{~nm}$ particle at $0.707 \mathrm{~V}_{\mathrm{rms}}$ is shown in D. The resulting temperature increase due to the presence of an electric field is shown in E. Figure F shows the simulated temperature gradient. Figure $\mathrm{G}$ shows the $\log _{10}$ magnitude of the DEP force at with a frequency of $10 \mathrm{MHz}$. The arrow plots in figure $\mathrm{G}$ are normalized to show direction only. Figure 41 shows the co-axial nano-probe. Figure 42 shows the triaxial nano-probe. Figure 43 shows a nano-probe geometry where the nano-probe is inset in a gold sleeve. Figure 44 shows a nano-probe geometry where the nano-probe extends beyond the gold sleeve. 


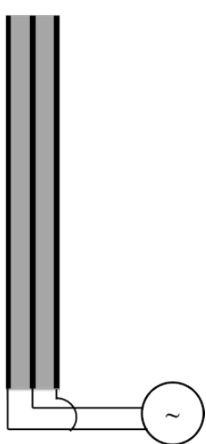

FIGURE 41A - Co-Axial Nano-Probe Illustration

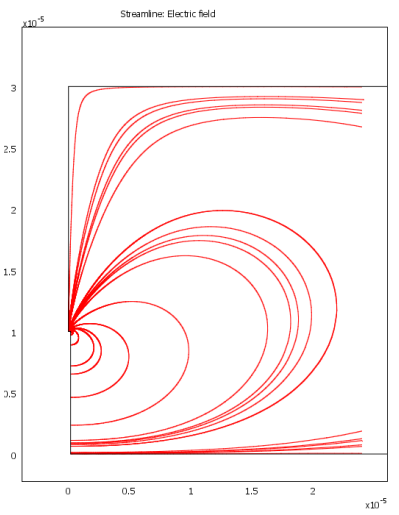

FIGURE 41B - Wide View of the Electric Field

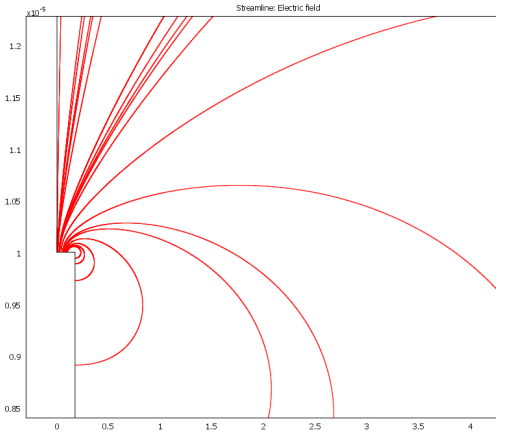

FIGURE 41C - Narrow View of the Electric Field

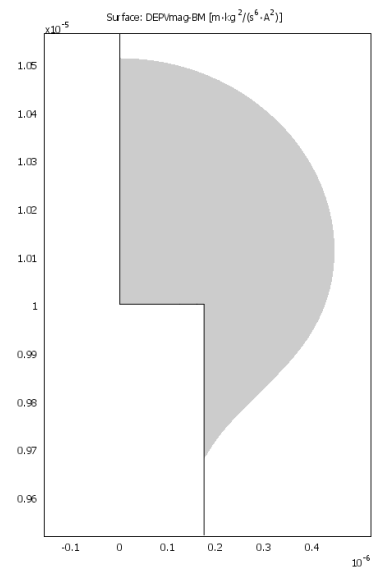

FIGURE 41D - Particle Capturing Radius

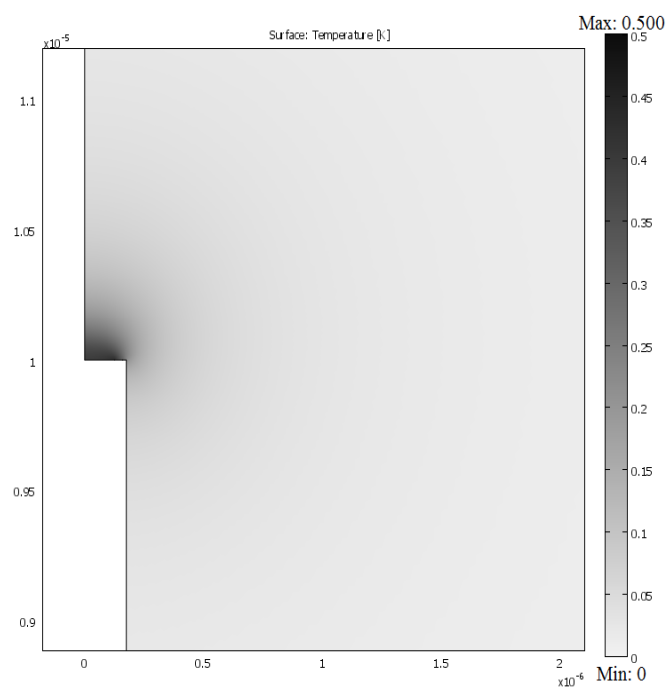

FIGURE 41E - Temperature Rise

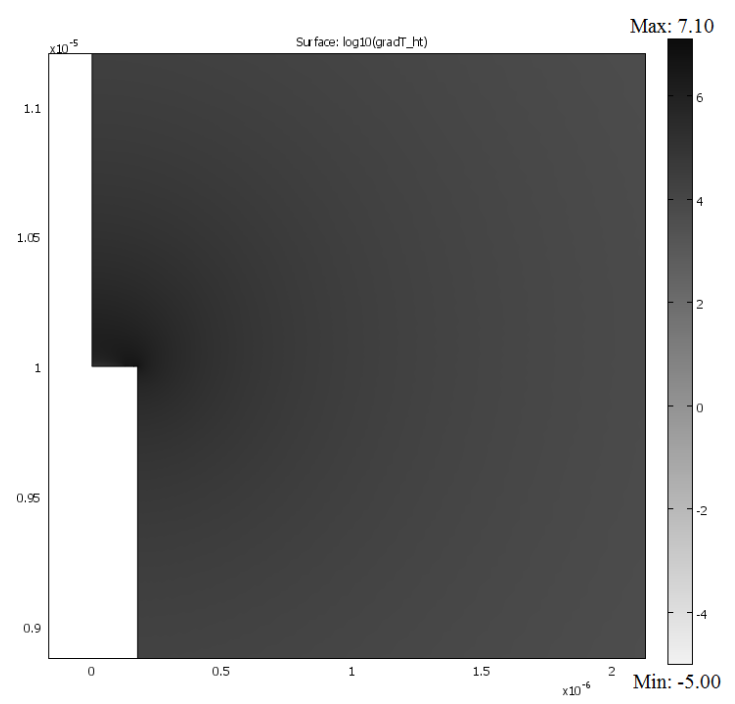

FIGURE 41F - $\log _{10}$ of the Temperature Gradient

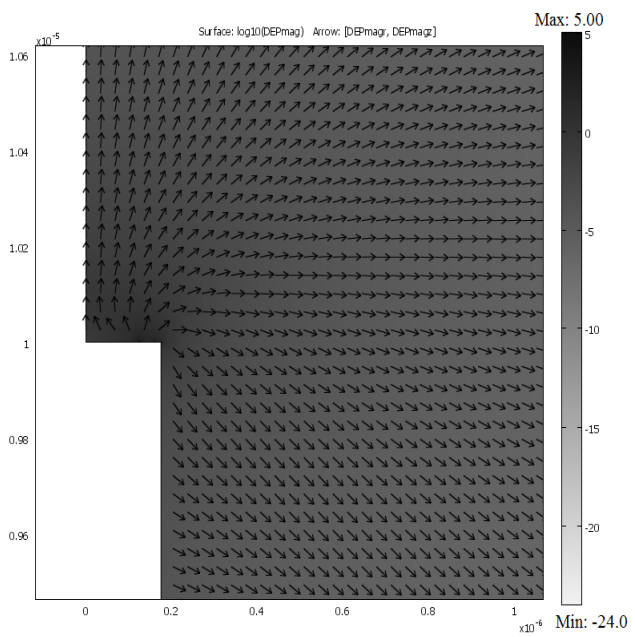

FIGURE $41 G-\log _{10}$ of the DEP Force with Direction 


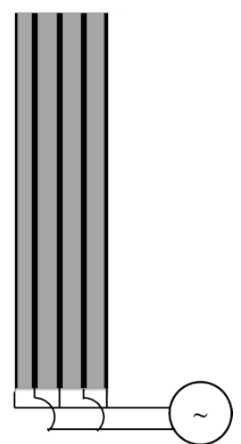

FIGURE 42A - Tri-Axial Nano-Probe Illustration

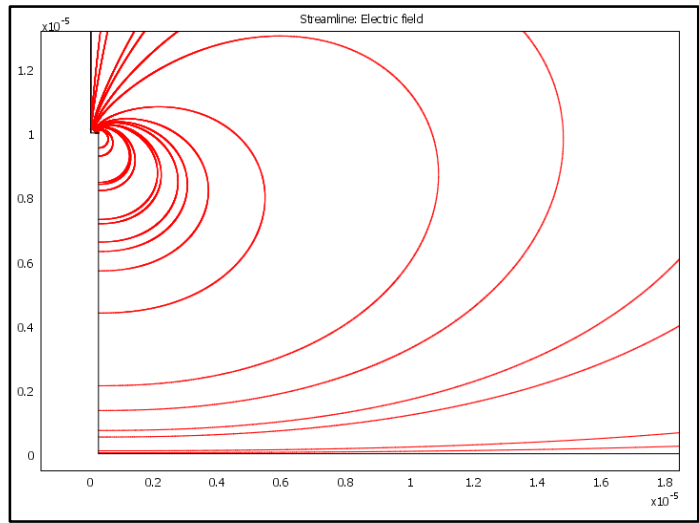

FIGURE 42B - Wide View of the Electric Field

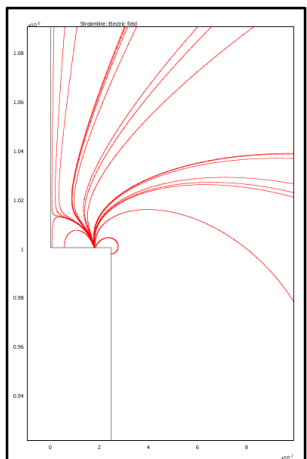

FIGURE 42C - Narrow View of the Electric Field

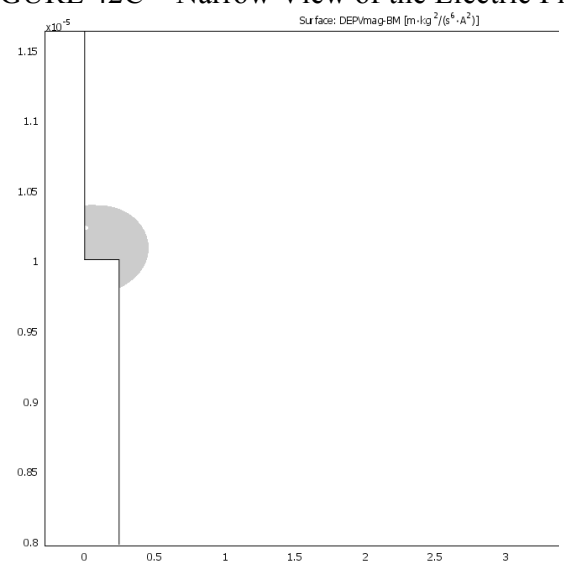

FIGURE 42D - Particle Capturing Radius

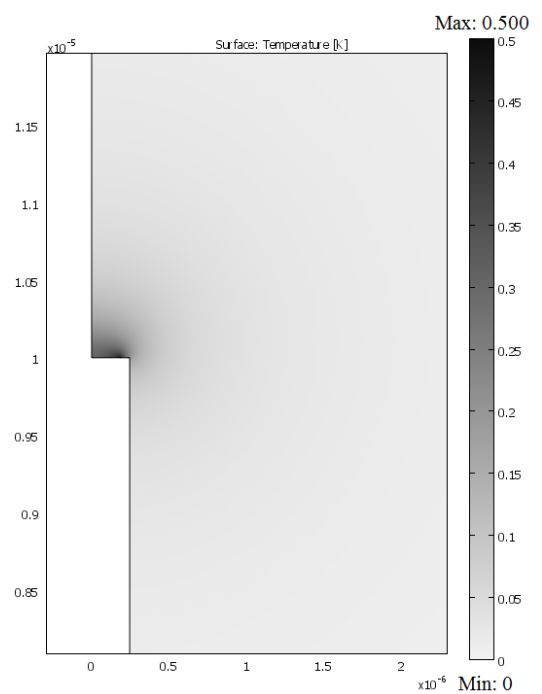

FIGURE 42E - Temperature Rise

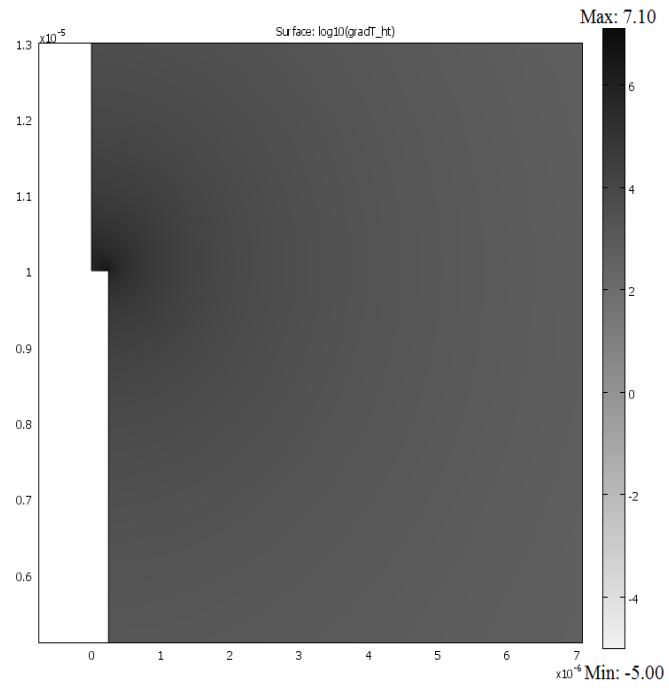

FIGURE $42 \mathrm{~F}-\log _{10}$ of the Temperature Gradient

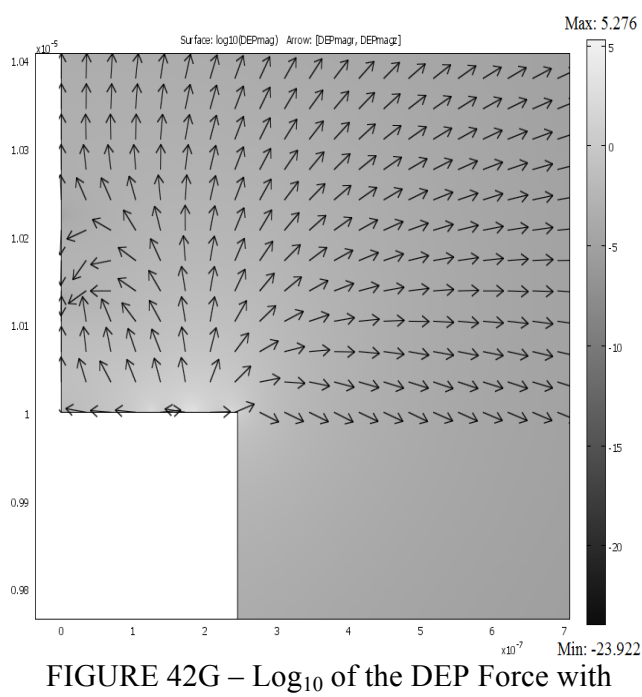
Direction 


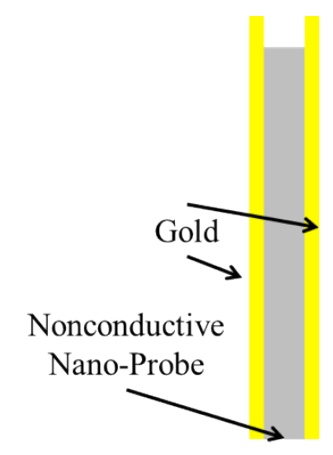

FIGURE 43A - Nano-Probe Inset in Gold Sleeve Illustration

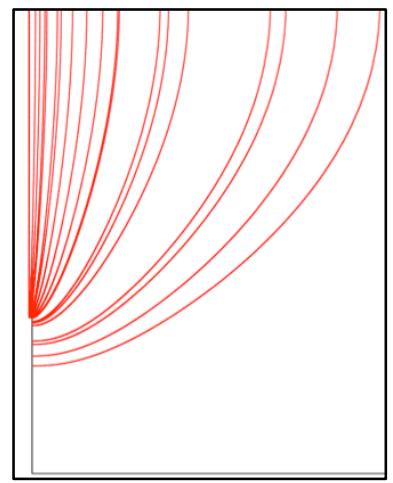

FIGURE 43B - Wide View of the Electric Field

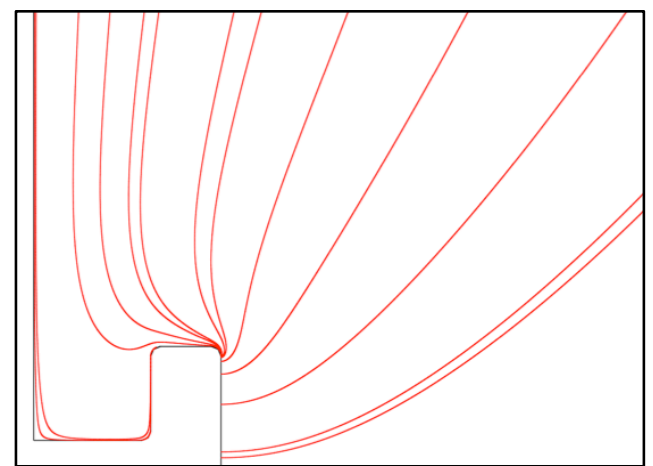

FIGURE 43C - Narrow View of the Electric Field

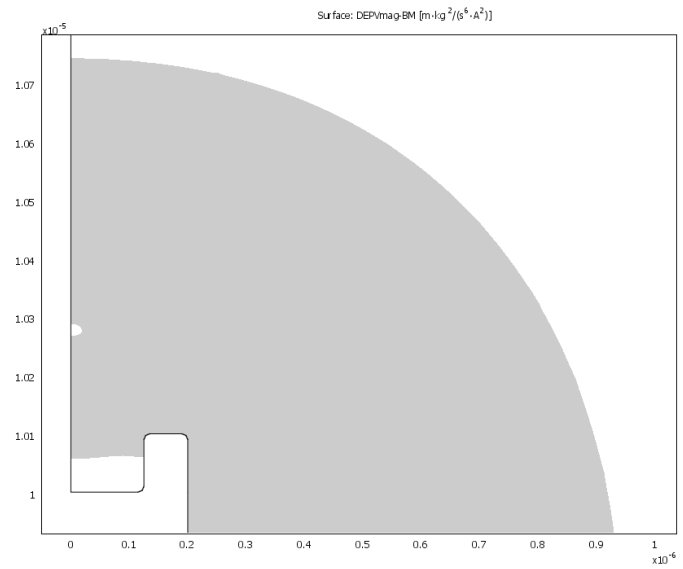

FIGURE 43D - Particle Capturing Radius

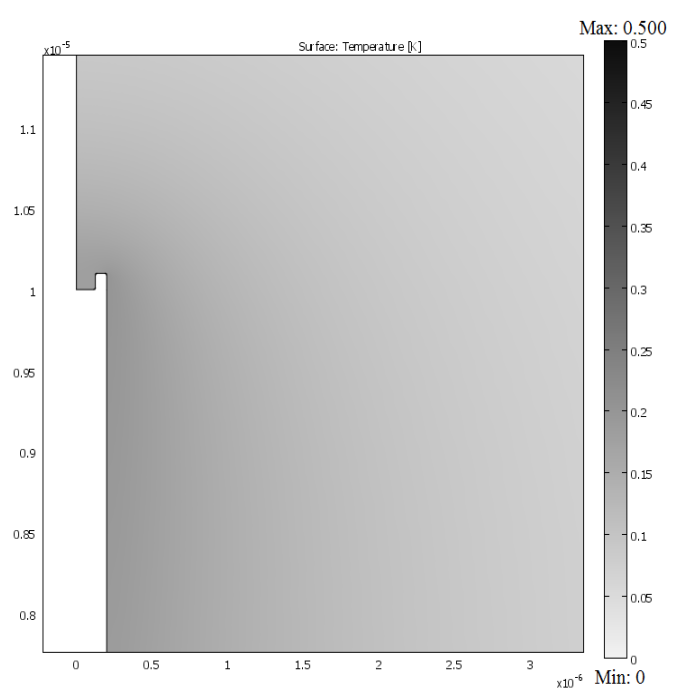

FIGURE 43E - Temperature Rise

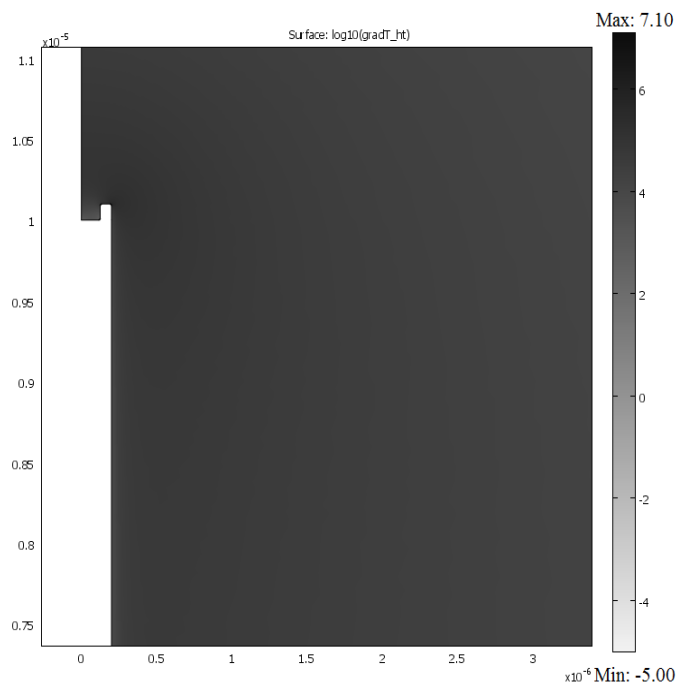

FIGURE 43F - $\log _{10}$ of the Temperature Gradient

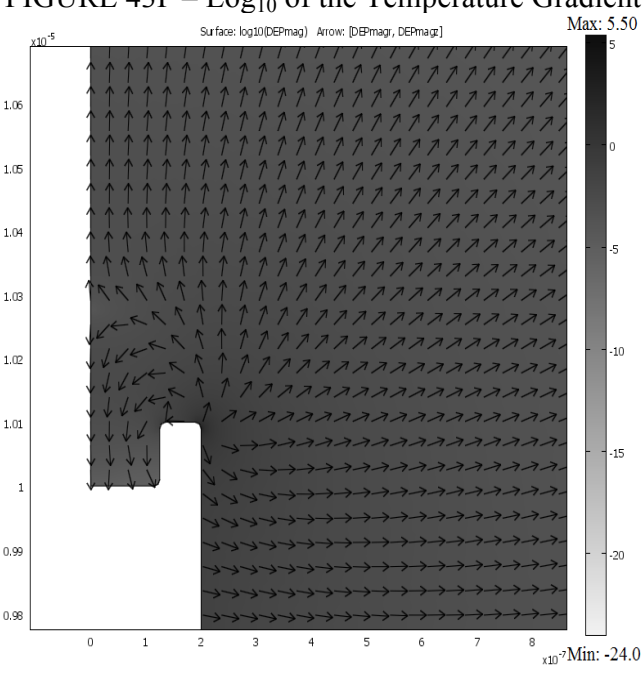

FIGURE $43 \mathrm{G}-\log _{10}$ of the DEP Force with Direction 


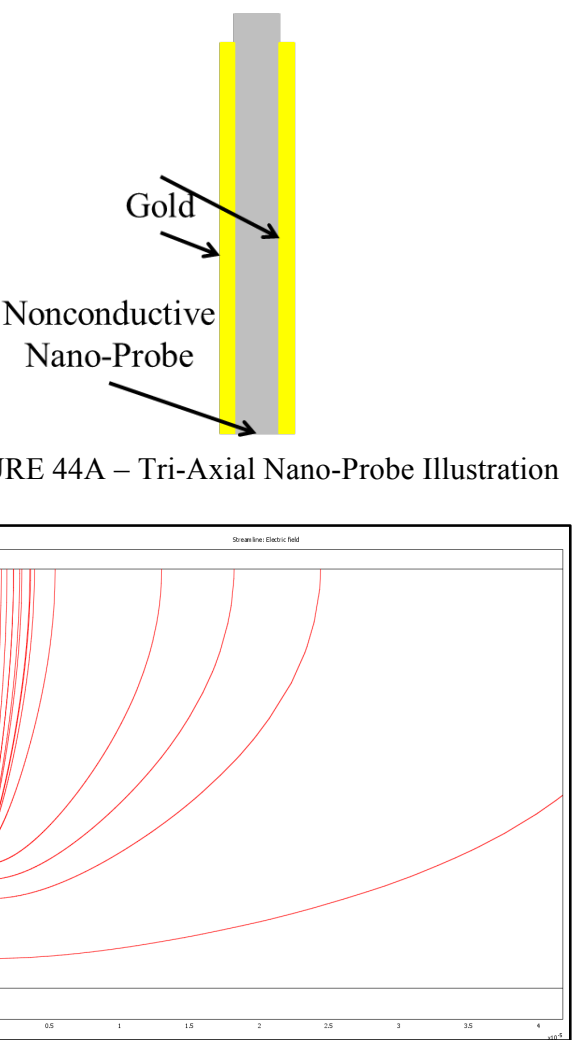

FIGURE 44B - Wide View of the Electric Field

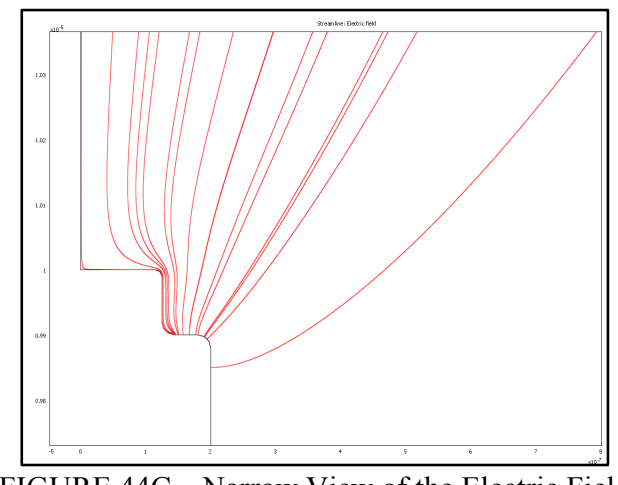

FIGURE 44C - Narrow View of the Electric Field

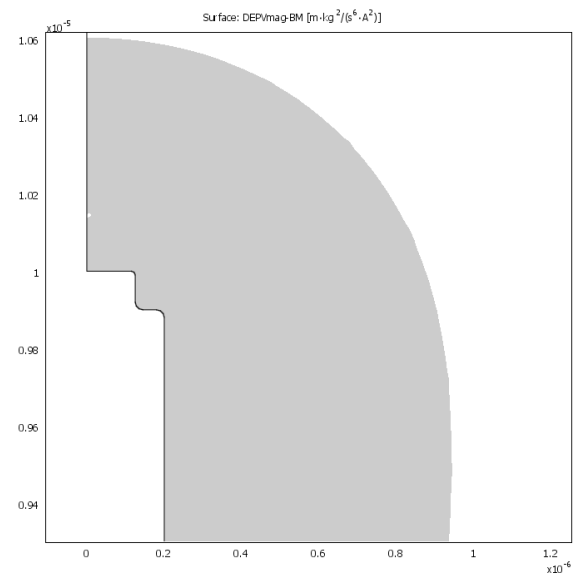

FIGURE 44D - Particle Capturing Radius

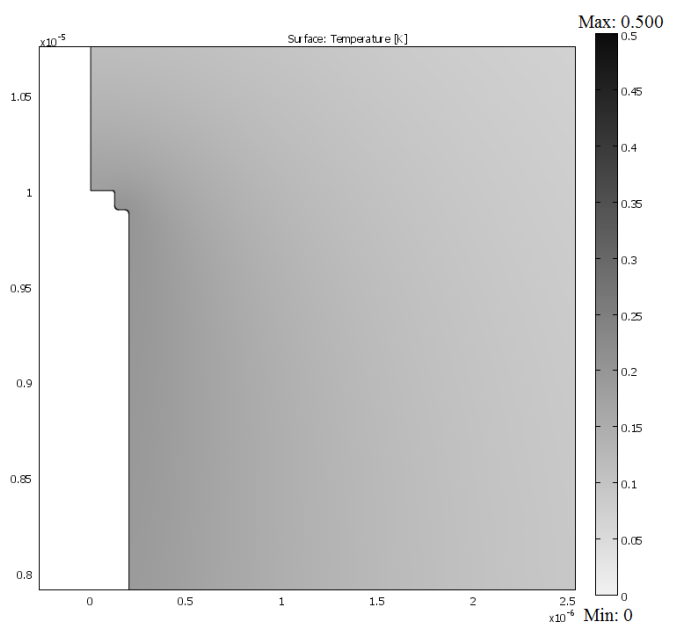

FIGURE 44E - Temperature Rise

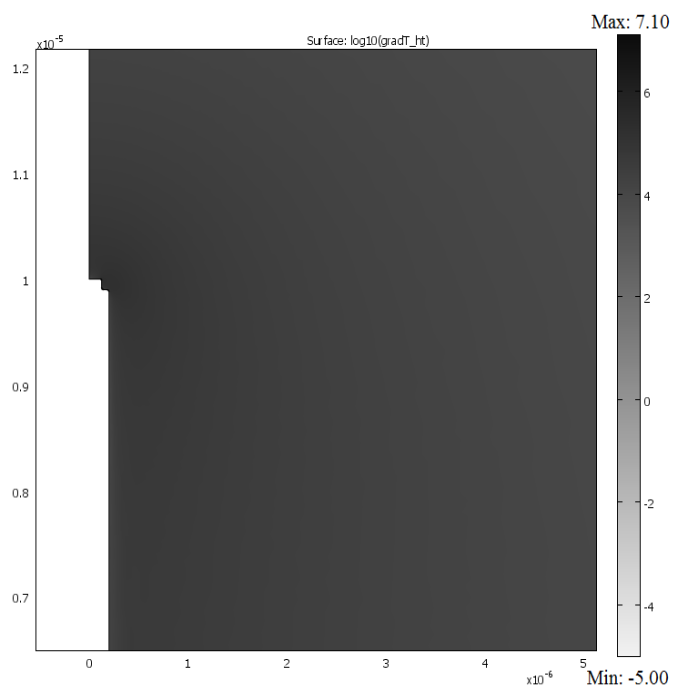

FIGURE $44 \mathrm{~F}-\log _{10}$ of the Temperature Gradient

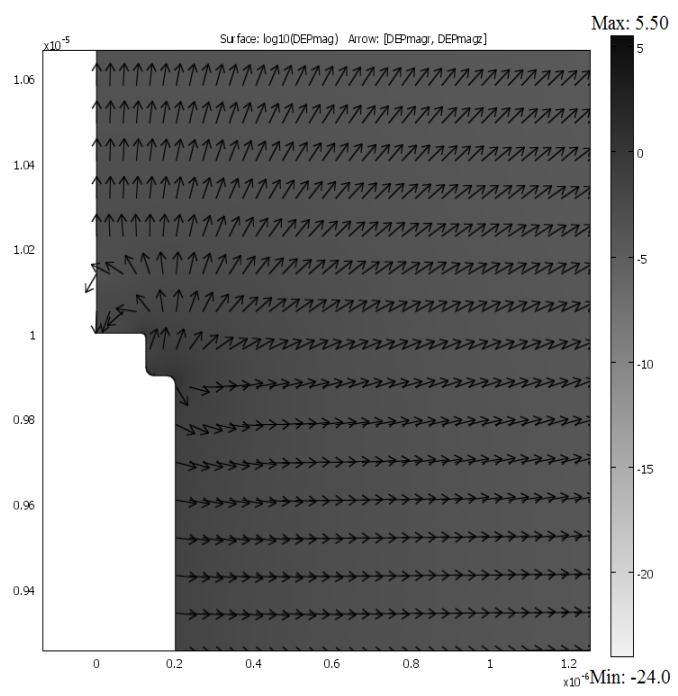

FIGURE $44 \mathrm{G}-\log _{10}$ of the DEP Force with Direction 


\section{Comparison of Nano-Probe Designs}

Table VI summarizes the data from the revised nano-probe designs.

TABLE VI

CHARACTERISTICS OF REVISED NANO-PROBES AT V $=1 \mathrm{~V}_{\mathrm{RMS}}$ AND $\sigma_{\mathrm{m}}=1 \mathrm{~S} / \mathrm{M}$

\begin{tabular}{|c|c|c|c|c|}
\hline \multicolumn{5}{|c|}{ Revised Nano-Probes } \\
\hline & Co-Axial & Tri-Axial & $\begin{array}{c}\text { Retracted Nano- } \\
\text { Probe }\end{array}$ & $\begin{array}{c}\text { Protruded Nano- } \\
\text { Probe }\end{array}$ \\
\hline$\nabla \mathrm{T}(\mathrm{K} / \mathrm{m})$ & $1.18 \mathrm{E}+07$ & $1.06 \mathrm{E}+07$ & $7.06 \mathrm{E}+05$ & $3.70 \mathrm{E}+05$ \\
\hline $\mathrm{T}_{\max }(\mathrm{K})$ & 0.425 & 0.463 & 0.202 & 0.202 \\
\hline $\log _{10}(\mathrm{DEP})$ & 4.667 & 5.276 & 3.461 & 1.658 \\
\hline $\begin{array}{c}\text { Trapping Radius } \\
(\mu \mathrm{m})\end{array}$ & 0.4 & 0.4 & 0.9 & 0.9 \\
\hline
\end{tabular}

The multi-axial nano-probes had much stronger DEP forces than the retracted or protruded nano-probes. However, the multi-axial nano-probes were simulated to have a temperature gradient two orders of magnitude higher than either of the other nano-probes, and the temperature rise was more than double the other nano-probe designs. The multiaxial nano-probes also had the smallest trapping radius.

The biggest concern in the data was the large temperature gradients. The DEP forces must be stronger than all of the other forces in the system in order to be observed. A large temperature gradient can create large amounts of electrothermal fluid flow. Electrothermal flow is the primary hindrance in high conductivity solutions. The applied voltage must be lowered to reduce the amount of ET fluid flow. However as the voltage is lowered, so is the region where particles experience DEP forces. In order to lower the voltage and still create a gradient in the electric field strong enough to influence particle motion, the nano-probe must become increasingly smaller in diameter. As the nano- 
probe decreases in diameter, the electrodes approximate the point-and-plane electrical system.

Based on the durability of the nano-probe and the gold jacket surrounding the nanoprobe, as well as the information of Table VI, the protruded nano-probe design would most likely be the best geometry for the desired nDEP trap. Durability would be a major concern with the shrouded nano-probe since the gold jacket would be tens of nanometers thick. 


\section{CONCLUSION}

The fabrication of silver gallium nano-probes is a novel approach for the creation of an electrode. The fabrication technique, initially discovered by Yazdanpanah et al. $[77,94]$, was modified in order to create the nano-probes on the sharp points of drawn out capillary tubes. The silver gallium nano-probes proved to be suitable for studying DEP forces in a three-dimensional droplet. $\mathrm{pDEP}$ and $\mathrm{nDEP}$ forces acted on polystyrene particles suspended in solutions of various conductivities when an AC signal was applied to the electrodes. These spherical polystyrene particles ranged from $200 \mathrm{~nm}$ to $1000 \mathrm{~nm}$ in diameter. The polystyrene particles were captured by the pDEP forces and were repelled by $\mathrm{nDEP}$ forces.

Strong DEP forces were generated by sharp gradients in the electric field resulting from an AC signal being applied to the sub-micrometer diameter nano-probe and the planar ITO coated coverslip. In most cases, the nano-probe was less than $40 \mu \mathrm{m}$ from the surface of the ITO coverslip. In the low conductivity solutions $(5.5 \mathrm{E}-8 \mathrm{~S} / \mathrm{m}$ and $2.5 \mathrm{E}-3$ $\mathrm{S} / \mathrm{m}$ ), both $\mathrm{pDEP}$ and nDEP forces were demonstrated. In the highest conductivity solution $(2.26 \mathrm{E}-1 \mathrm{~S} / \mathrm{m})$ only $\mathrm{nDEP}$ was shown.

The magnitude of the other electrokinetic forces in the system increased with the

conductivity of the suspending medium. The particle motion in the highest conductivity 
solution $(2.26 \mathrm{E}-1 \mathrm{~S} / \mathrm{m})$ was primarily dominated by the electrothermal fluid flow due to the large temperature gradient generated near the end of the nano-probe. When an AC signal with a high frequency (near $50 \mathrm{MHz}$ ) was applied, nDEP forces were able to dominate over the electrothermal motion in the droplet; electrothermal fluid velocity is dependent on the applied frequency of the signal and decreases at higher frequencies.

The nano-probe was also capable of capturing gold nano-particles as small as 5 $\mathrm{nm}$ in diameter with pDEP forces at voltages less than $1 \mathrm{~V}_{\mathrm{rms}}$. The non-circular gold nano-particles assembled in a fractal pattern and subsequently fused together to form semi-rigid structures on the nano-probe. Fractal patterns formed due to the localized, increased electric field gradients caused by the adhered nano-particles themselves, attracting additional gold nano-particles with pDEP. These structures stayed attached to the nano-probe without the presence of the applied AC signal. 


\section{RECOMMENDATIONS}

The future goal of the project is to create a co-axial nano-probe capable of using pDEP and nDEP forces within a cellular structure to attract and repel sub-cellular entities. However, due to the high conductivity of the cytoplasm [44], pDEP capture will most likely not be possible. The next step is to continue work to fabricate a nano-probe with a new geometry, possibly a tri-axial nano-probe, or a nano-probe similar to those found in Figures $43 \mathrm{~A}$ and/or $44 \mathrm{~A}$. These nano-probes create a nDEP trap where particles are captured without physically contacting the nano-probe.

With or without the new nDEP nano-probe, studies should be done regarding the effects of $\mathrm{pDEP}$ on the sub-cellular entities in a low conductivity. Visualization of the sub-cellular entities can quite difficult without the aid of a fluorescent protein bonded to entities, so different optical methods should be tested. If the sub-cellular entities will not fluoresce, then additional microscopic techniques, such as DIC or dark-field microscopy, should be tested to visualize subcellular entities.

The capture of sub-cellular entities by pDEP forces is expected in a low conductivity medium. When polystyrene particles were suspended in a low conductivity medium and a low frequency signal was applied, the number of particles captured with pDEP forces increased with time. Similarly, pDEP capture should be easier to visualize 
if the AC signal is applied to the nano-probe for a long period of time. pDEP forces acting on the sub-cellular entities will most likely be the easiest to visualize. Unless there is a high concentration of sub-cellular entities, pure nDEP will be difficult to show experimentally. The nDEP trap created by the different nano-probe geometries would likely have a similar effect as that of pDEP.

If capture of the sub-cellular entities is achieved, the entities should be analyzed in order to characterize the specific entity captured. This analysis would take place based on the electrical characteristics of the sub-cellular entities. For example, the frequency of the signal could be varied to determine the conductivity of the sub-cellular entities. This process should be repeated for several different sub-cellular entities. Once several subcellular entities are sufficiently characterized, the conductivity of the medium should be increased to approximate that of the cytoplasm in a cell, $1 \mathrm{~S} / \mathrm{m} \mathrm{[44].} \mathrm{As} \mathrm{the} \mathrm{conductivity}$ is gradually increased to this value, the change in the surface charge, and therefore the conductance, of the sub-cellular entities should be closely monitored. It would be reasonable to expect only nDEP forces to be capable of dominating the system when the suspending medium approaches $1 \mathrm{~S} / \mathrm{m}$, and at very high frequencies.

If the multi-axial or nDEP nano-probes are created, and the capture or repulsion of the sub-cellular entities is shown in experiments outside of a cell, then the next logical step would be to insert the nano-probe into the cell and apply a signal to the nano-probe. If the sub-cellular entities are characterized in a suspending medium with a high conductivity, the nDEP nano-probes should allow the organelles to be manipulated with relative ease. 


\section{REFERENCES}

1. Pohl, H., Dielectrophoresis 1978, Cambridge: Cambridge University Press. 579.

2. Jones, T.B., Electromechanics of Particles1995, New York: Cambridge University Press. 265.

3. $\quad$ Feynman, R.P., There's Plenty of Room at the Bottom, 1959.

4. Yazdanpanah, M.M., Near Room Temperature of Self-Assembly of Nanostructures by Reaction of Gallium with Metal Thin Films, in Electrical and Computer Engineering2006, University of Louisville. p. 239.

5. Khondaker, S.I., K. Luo, and Z. Yao, The fabrication of single-electron transistors using dielectrophoretic trapping of individual gold nanoparticles. Nanotechnology, 2010. 21(9): p. 095204.

6. Kim, H.J., et al., Microfluidic device to separate micro-beads with various fluorescence intensities. Sensors and Actuators B: Chemical, 2011. 160(1): p. 1536-1543.

7. Ye, Y., et al., Thermophoretic effect of particle deposition on a free standing semiconductor wafer in a clean room. Journal of Aerosol Science, 1991. 22(1): p. 63-72.

8. Einstein, A., On the Moleculare-Kinetic Theory of Heat Required by the Motion of Particles Suspended in Stationary Liquids. Annalen der Physik, 1905. 322(8): p. 549-560.

9. Castellanos, A., et al., Electrohydrodynamics and dielectrophoresis in microsystems: scaling laws. Journal of Physics D: Applied Physics, 2003. 36: p. 14.

10. Strickland, R.D., Electrophoresis. Analytical Chemistry, 1966. 38(5): p. 99-130.

11. Muller, T., et al., Microdevice for Cell and Particle Seperation using Dielectrophoretic Field-Flow Fractionation. Liq. Chrom. \& Rel. Technol., 2000. 23(1): p. 12.

12. Broche, L., et al., Early detection of oral cancer - Is dielectrophoresis the answer? Oral Oncology, 2007. 43: p. 5.

13. Mulhall, H.J., et al., Cancer, pre-cancer and normal oral cells distinguished by dielectrophoresis. Anal Bioanal Chem, 2011. 401(8): p. 2455-63.

14. Lapizco-Encinas, B.H. and M. Rito-Palomares, Dielectrophoresis for the manipulation of nanobioparticles. Electrophoresis, 2007. 28(24): p. 4521-38.

15. Thomas, R.S., et al., Trapping single human osteoblast-like cells from a heterogeneous population using a dielectrophoretic microfluidic device. Biomicrofluidics, 2010. 4(2).

16. Thomas, R.S., H. Morgan, and N.G. Green, Negative DEP traps for single cell immobilisation. Lab Chip, 2009. 9(11): p. 1534-40.

17. Ying, L., et al., Frequency and Voltage Dependence of the Dielectrophoretic Trapping of Short Lenghts of DNA and dCTP in a Nanopipette. Biophysical Journal, 2004. 86: p. 9.

18. Zheng, L., J.P. Brody, and P.J. Burke, Electronic manipulation of DNA, proteins, and nanoparticles for potential circuit assembly. Biosens Bioelectron, 2004. 20(3): p. 606-19. 
19. Hsieh, B.-C., et al., Pencil lead microelectrode and the application on cell dielectrophoresis. Electrochimica Acta, 2011. 56(27): p. 9916-9920.

20. Kostner, S., et al., Guided Dielectrophoresis: A Robust Method for Continuous Particle and Cell Seperation. IEEE Sensors Journal, 2010. 10(9): p. 7.

21. Kuzyk, A., Dielectrophoresis at the nanoscale. Electrophoresis, 2011.

22. Salonen, E., et al., Dielectrophoresis of nanocolloids: a molecular dynamics study. Eur Phys J E Soft Matter, 2005. 18(2): p. 133-42.

23. Zhang, C., et al., Dielectrophoresis for manipulation of micro/nano particles in microfluidic systems. Anal Bioanal Chem, 2010. 396(1): p. 401-20.

24. Abbas, A., et al., Freezing the self-assembly process of gold nanocrystals. Chem Commun (Camb), 2012. 48(11): p. 1677-9.

25. Barsotti, R.J., Jr., et al., Assembly of metal nanoparticles into nanogaps. Small, 2007. 3(3): p. 488-99.

26. Dadosh, T., et al., Measurement of the conductance of single conjugated molecules. Nature, 2005. 436(7051): p. 677-80.

27. Du, F., et al., Dielectrophoretic Gold Particle Separation. Separation Science and Technology, 2008. 43(15): p. 3842-3855.

28. Gierhart, B.C., et al., Frequency Dependence of Gold Nanoparticle Superassembly by Dielectrophoresis. Langmuir, 2007. 23: p. 7.

29. Khondaker, S.I. and Z. Yao, Fabrication of nanometer-spaced electrodes using gold nanoparticles. Applied Physics Letters, 2002. 81(24): p. 4613.

30. Kumar, S., S.-H. Yoon, and G.-H. Kim, Bridging the nanogap electrodes with gold nanoparticles using dielectrophoresis technique. Current Applied Physics, 2009. 9(1): p. 101-103.

31. Li, M.L., et al., Dielectrophoretic Assembly of $2 \mathrm{~nm}$ Gold Particles for Nanosensing Applications, in IEEE International Conference on Nano/Micro Engineered and Molecular Systems2010: Xiamen, China. p. 5.

32. Zheng, L., Self-assembled gold nanowires from nanoparticles: an electronic route towards DNA nanosensors. 2004. 5515: p. 117-124.

33. Kostner, S., et al., Guided Dielectrophoresis: A Robust Method for Continuous Particle and Cell Separation. IEEE Sensors Journal, 2010. 10(9): p. 7.

34. Moffitt, J.R., et al., Recent advances in optical tweezers. Annu Rev Biochem, 2008. 77: p. 205-28.

35. Muller, T., et al., Microdevice for Cell and Particle Separation Using Dielectrophoretic Field-Flow Fractionation. Journal of Liquid Chromatography \& Related Technologies, 2000. 23(1): p. 15.

36. O'Konski, C.T., Electric Properties of Macromolecules v. Theory of Ionic Polarization in Polyelectrolytes. Journal of Physical Chemistry, 1960. 64: p. 15.

37. Sridharan, S., et al., Joule heating effects on electroosmotic flow in insulatorbased dielectrophoresis. Electrophoresis, 2011.

38. Tsai, S.L., et al., Experimental study of dielectrophoresis and liquid dielectrophoresis mechanisms for particle capture in a droplet. Electrophoresis, 2011. 32(11): p. 1337-47.

39. Wang, D. and H. Mohwald, Template-directed colloidal self-assembly - the route to 'top-down' nanochemical engineering. Journal of Materials Chemistry, 2004. 14: p. 10 . 
40. Wang, D., M. Sigurdson, and C.D. Meinhart, Experimental analysis of particle and fluid motion in ac electrokinetics. Experiments in Fluids, 2004. 38(1): p. 110 .

41. Zhang, H., et al., Directing the self-assembly of nanocrystals beyond colloidal crystallization. Phys Chem Chem Phys, 2006. 8(28): p. 3288-99.

42. Zheng, C., Investigation of Dielectrophoresis and Its Applications in Micro/nano Fluidics, Electronics and Fabrications, in School of Electrical and Computer Engineering2010, RMIT University: Melboune, Australia. p. 202.

43. Zheng, L., et al., Manipulating Nanoparticles in Solution with Electrically Contacted Nanotubes Using Dielectrophoresis. Langmuir, 2004. 20: p. 8.

44. Cohn, R.W. and S.J. Williams, Dielectrophoretic nanoneedles for trapping and characterization of sub-cellular entities, 2011.

45. Abramowitz, M., Microscope: Basics and Beyond, 2003.

46. Srivastava, R. and K.N. Khanna, Stokes-Einstein in Two- and Three-Dimensional Fluids. J. Chem. Eng. Data, 2009. 54(5): p. 4.

47. Xuan, X., Joule heating in electrokinetic flow. Electrophoresis, 2008. 29(1): p. 3343.

48. Muller, T., et al., Trapping of micrometre and sub-micrometre particles by highfrequency electric fields and hydrodynamic forces. J. Phys. D: Appl. Phys., 1996. 29: p. 9.

49. N.G. Green, H.M., Dielectorphoretic seperation of nano-particles. J. Phys. D: Appl. Phys., 1997. 30.

50. Gonzalez, A., et al., Electrothermal flows generated by alternating and rotating electric fields in microsystems. Journal of Fluid Mechanics, 2006. 564: p. 415.

51. Ramos, A., et al., Ac electrokinetics: a review of forces in microelectrode structures. Journal of Physics D: Applied Physics, 1998. 31: p. 16.

52. Perch-Nielsen, I.R., N.G. Green, and A. Wolff, Numerical simulation of travelling wave induced electrothermal fluid flow. Journal of Physics D: Applied Physics, 2004. 37(16): p. 2323-2330.

53. Green, N.G., et al., Electrothermally induced fluid flow on microelectrodes. Journal of Electrostatics, 2001. 53: p. 17.

54. Zhang, C., Investigation of Dielectrophoresis and Its Applications in Micro/nano Fluidics, Electronics and Fabrications, in Electronics2010, RMIT University. p. 202.

55. Gierhart, B.C., et al., Frequency Dependence of Gold Nanoparticle Superassembly by Dielectrophoresis. Langmuir, 2007. 23: p. 6.

56. Hong, S.H., et al., Controllable Capture of Au Nano-Particles by Using Dielectrophoresis. Journal of the Korean Physical Society, 2004. 45(December): p. 665-668.

57. Huang, Y. and R. Pethig, Electrode design for negative dielectrophoresis. Measurment Science and Engineering, 1991. 2: p. 4.

58. Lee, H.J., et al., Simple and rapid preparation of vertically aligned gold nanoparticle arrays and fused nanorods in pores of alumina membrane based on positive dielectrophoresis. Sensors and Actuators B: Chemical, 2009. 136(2): p. 320-325. 
59. Na, J.S., et al., Conduction mechanisms and stability of single molecule nanoparticle/molecule/nanoparticle junctions. Nanotechnology, 2007. 18(3): p. 035203.

60. Winter, W.T. and M.E. Welland, Dielectrophoresis of non-spherical particles. Journal of Physics D: Applied Physics, 2009. 42(4): p. 045501.

61. Zheng, L., et al., Towards Single Molecule Manipulation with Dielectrophoresis Using Nanoelectrodes. IEEE.

62. Zheng, L., et al., Manipulating Nanoparticles in Solution with Electrically Contacted Nanotubes Using Dielectrophoresis. Langmuir, 2004. 20: p. 7.

63. Brown, K.A. and R.M. Westervelt, Triaxial AFM probes for noncontact trapping and manipulation. Nano Lett, 2011. 11(8): p. 3197-201.

64. Castellanos, A., et al., Electrohydrodynamics and dielectrophoresis in microsystems: scaling laws. J. Phys. D: Appl. Phys., 2003. 36: p. 12.

65. Lapizco-Encinas, B.H. and F. Foret, Dielectrophoresis 2011 - Part I. Electrophoresis, 2011. 32: p. 1.

66. Lapizco-Encinas, B.H. and F. Foret, Dielectrophoresis 2011 - Part II. Electrophoresis, 2011. 32: p. 1.

67. Brown, K.A., J.A. Aguilar, and R.M. Westervelt, Coaxial atomic force microscope tweezers. Applied Physics Letters, 2010. 96(12): p. 123109.

68. Park, J.-K., et al., Morphology control and integration of the carbon nanotube tip for AFM. Current Applied Physics, 2006. 6: p. e220-e223.

69. Lee, H.W., et al., Nanoscale fabrication of a single multiwalled carbon nanotube attached atomic force microscope tip using an electric field. Review of Scientific Instruments, 2005. 76(4): p. 046108.

70. Cetin, B. and D. Li, Effect of Joule heating on electrokinetic transport. Electrophoresis, 2008. 29(5): p. 994-1005.

71. Ramos, A., et al., Ac electrokinetics: a review of forces in microelectrode structures. J. Phys. D: Appl. Phys., 1998. 31: p. 15.

72. Evenhuis, C.J. and P.R. Haddad, Joule heating effects and the experimental determination of temperature during CE. Electrophoresis, 2009. 30(5): p. 897909.

73. Sabah, E., et al., Zeta Potentials of Sepiolite Suspensions in Concentrated Monovalent Electrolytes. Separation Science and Technology, 2007. 42(10): p. 2275-2288.

74. Park, S., et al., Continuous dielectrophoretic bacterial separation and concentration from physiological media of high conductivity. Lab on a Chip, 2011. 11(17).

75. Wright, M.H. and A.M. James, The surface conductivity at the polystyrene-latex particle/electrolyte interface. Colloid \& Polymer Science, 1973. 251(10): p. 745751.

76. Reppert, P., Frequency-Dependent Electroosmosis. Journal of Colloid and Interface Science, 2002. 254(2): p. 372-383.

77. Yazdanpanah, M.M., et al., Selective self-assembly at room temperature of individual freestanding $\mathrm{Ag}[\mathrm{sub} 2] \mathrm{Ga}$ alloy nanoneedles. Journal of Applied Physics, 2005. 98(7): p. 073510. 
78. Yazdanpanah, M.M., et al., Selective self-assembly at room temperature of individual freestanding Ag2Ga alloy nanoneedles. Journal of Applied Physics, 2005. 98(7): p. 073510.

79. Jiang, X.C., et al., Self-assembly of particles: some thoughts and comments. Journal of Materials Chemistry, 2011. 21(42): p. 16797.

80. Biedermann, L.B., et al., Flexural vibration spectra of carbon nanotubes measured using laser Doppler vibrometry. Nanotechnology, 2009. 20(3): p. 035702.

81. Dobrokhotov, V.V., et al., Visual force sensing with flexible nanowire buckling springs. Nanotechnology, 2008. 19(3): p. 035502.

82. Yang, C.Y. and U. Lei, Dielectrophoretic force and torque on an ellipsoid in an arbitrary time varying electric field. Applied Physics Letters, 2007. 90(15): p. 153901.

83. Christensen, T.M.; Available from: http://www.uccs.edu/ tchriste/courses/PHYS549/549lectures/sputtertech.html.

84. Lowery, A., et al., Immunonanoshells for targeted photothermal ablation of tumor cells. International Journal of Nanomedicine, 2006. 1: p. 5.

85. Arnold, W.M., H.P. Schwan, and U. Zimmermann, Surface Conductance and Other Properites of Latex Particles Measured by Electrorotataion. Journal of Physical Chemistry, 1987. 91: p. 6.

86. Dong, Z., Dielectrophoresis (DEP) Based Microfluidic Particle Seperator.

87. Kretschmer, R. and W. Fritzsche, Pearl Chain Formation of Nanoparticles in Microelectrode Gaps by Dielectrophoresis. Langmuir, 2004. 20: p. 5.

88. Amlani, I., et al., An approach to transport measurements of electronic molecules. Applied Physics Letters, 2002. 80(15): p. 2761.

89. Evoy, S., et al., Dielectrophoretic assembly and integration of nanowire devices with functional CMOS operating circuitry. Microelectronic Engineering, 2004. 75(1): p. 31-42.

90. Green, N.G. and H. Morgan, Dielectrophoretic separation of nano-particles. Journal of Physics D: Applied Physics, 1997. 30: p. 4.

91. Hong, S.H., et al., Controllable Capture of Au Nano-Particles Using Dielectrophoresis. Journal of the Korean Physical Society, 2004. 45: p. 4.

92. Muller, T., et al., Trapping of micrometre and sub-micrometre particles by highfrequency electric fields and hydrodynamic forces. Journal of Physics D: Applied Physics, 1996. 29: p. 9.

93. Ying, L., et al., Frequency and Voltage Dependence of the Dielectrophoretic Trapping of Short Lengths of DNA and dCTP in a Nanopipette. Biophysical Journal, 2004. 86(February): p. 10.

94. Yazdanpanah, M.M., Near Room Temperature Self-Assembly of Nanostructures By Reaction of Gallium With Metal Thin Films, in Department of Electrical and Computer Engineering2006, University of Louisville: Louisville, Ky. p. 239. 
VITA

Nicholas R. Wood, EIT

8405 Easton Commons Dr. Apt. C

Louisville, KY 40242

(270) 256-5362

NRWood01@gmail.com

\section{EDUCATION}

University of Louisville

Bachelor of Science, May 2011

Major: Mechanical Engineering

GPA: $3.704 / 4.0$

\section{University of Louisville}

Master of Engineering, Expected May 2012

Major: Mechanical Engineering

GPA: 4.0/4.0

\section{WORK EXPERIENCE}

Research Assistant, August 2011-May 2012

University of Louisville, Louisville, KY

- Assisted Professors in optimizing the manufacture of coaxial nano-needles

Engineering Co-op, May 2010-August 2010

Marathon Petroleum Company, Robinson, IL

- Wrote and presented decision support packages for project approval

- Completed detailed design for several projects

- Issued multiple construction packages for projects

Engineering Co-op, August 2009-December 2009

Marathon Petroleum Company, Robinson, IL

- Responsible for the Remote Asset Data Analysis and Response program

- Achieved over $\$ 40,000$ savings

Engineering Co-op, January 2009-May 2009

Marathon Petroleum Company, Robinson, IL

- Field Coordinator for a project involving different methods of underground exploratory work

- Reviewed and approved contractor budget and final expense report

Math Tutor, August 2008-December 2008

University of Louisville, Louisville, KY

- Tutored students in classes ranging from pre-algebra to differential equations 


\section{PRESENTATIONS}

- KY Nanotechnology Symposium

○ Oral Presentation - Trapping of Nanoparticles with Dielectrophoretic Nanoneedles

- Poster Presentation - Trapping of Nanoparticles with Dielectrophoretic Nanoneedles

\section{SKILLS}

- ASME B31.3 Certified

\section{HONORS AND ACTIVITIES}

- University of Louisville Trustee's Scholarship, President's Scholar

- Dean's List, 3 Semesters

- Dean's Scholar, 2 Semesters

- Honors Program Graduate

- Tau Beta Pi, National Engineering Honors Society

- Pi Tau Sigma, Mechanical Engineering Honors Society 